\title{
Gravitational waves from spinning black hole-neutron star binaries: dependence on black hole spins and on neutron star equations of state
}

\author{
Koutarou Kyutoku, Hirotada Okawa, Masaru Shibata \\ Yukawa Institute for Theoretical Physics, Kyoto University, Kyoto 606-8502, Japan \\ Keisuke Taniguchi \\ Graduate School of Arts and Sciences, University of Tokyo, Komaba, Meguro, Tokyo 153-8902, Japan
}

(Dated: October 30, 2018)

\begin{abstract}
We study the merger of black hole-neutron star binaries with a variety of black hole spins aligned or antialigned with the orbital angular momentum, and with the mass ratio in the range $M_{\mathrm{BH}} / M_{\mathrm{NS}}=$ 2-5, where $M_{\mathrm{BH}}$ and $M_{\mathrm{NS}}$ are the mass of the black hole and neutron star, respectively. We model neutron-star matter by systematically parametrized piecewise polytropic equations of state. The initial condition is computed in the puncture framework adopting an isolated horizon framework to estimate the black hole spin and assuming an irrotational velocity field for the fluid inside the neutron star. Dynamical simulations are performed in full general relativity by an adaptive-mesh refinement code, SACRA. The treatment of hydrodynamic equations and estimation of the disk mass are improved. We find that the neutron star is tidally disrupted irrespective of the mass ratio when the black hole has a moderately large prograde spin, whereas only binaries with low mass ratios, $M_{\mathrm{BH}} / M_{\mathrm{NS}} \lesssim 3$, or small compactnesses of the neutron stars bring the tidal disruption when the black hole spin is zero or retrograde. The mass of the remnant disk is accordingly large as $\gtrsim 0.1 M_{\odot}$, which is required by central engines of short gamma-ray bursts, if the black hole spin is prograde. Information of the tidal disruption is reflected in a clear relation between the compactness of the neutron star and an appropriately defined "cutoff frequency" in the gravitational-wave spectrum, above which the spectrum damps exponentially. We find that the tidal disruption of the neutron star and excitation of the quasinormal mode of the remnant black hole occur in a compatible manner in high mass-ratio binaries with the prograde black hole spin. The correlation between the compactness and the cutoff frequency still holds for such cases. It is also suggested by extrapolation that the merger of an extremely spinning black hole and an irrotational neutron star binary does not lead to the formation of an overspinning black hole.
\end{abstract}

PACS numbers: 04.25.D-, 04.30.-w, 04.40.Dg

\section{INTRODUCTION}

Coalescing binaries composed of a black hole $(\mathrm{BH})$ and/or a neutron star (NS) are among the most promising sources of gravitational waves for ground-based laser-interferometric gravitational-wave detectors such as LIGO [1] and VIRGO [2]. Detections of gravitational waves will be accomplished in a decade to come by planned next-generation detectors such as advanced LIGO, advanced VIRGO, and LCGT [3]. Because gravitational waves are much more transparent to the absorption and scattering by the material than electromagnetic waves and even neutrinos are, gravitational-wave astronomy is expected to become a powerful and unique way to observe strongly gravitating phenomena in our Universe. Among such phenomena, the merger of a BH-NS binary plays an important role to investigate properties of the NS such as the radius and the equation of state (EOS) of a high-density nuclear matter [4 9]. An important constraint on the EOS is obtained from detection of a $1.97 \pm 0.04 M_{\odot} \mathrm{NS}$, which is the most massive NS currently known 10, by recent pulsar-timing observation. However, we still do not know the realistic EOS of the NS because there is no robust measurement of the NS radius [11]. To constrain the NS radius and EOS by observing gravitational waves from the BH-NS binary, we have to prepare accurate theoretical templates of gravitational waveforms employing a wide variety of the NS EOSs and other physical parameters. For this purpose, numerical relativity is the unique approach.

The merger of a BH-NS binary is also an important target for the astrophysical study because it is a potential candidate for the progenitor of short-hard gammaray bursts (GRBs) in the so-called merger scenario (see Refs. [12, 13] and references therein for reviews). If a NS is tidally disrupted during the merger of a BH-NS binary, a system composed of a spinning $\mathrm{BH}$ and a hot, massive accretion disk of $\gtrsim 0.01 M_{\odot}$ may be formed. Such an outcome could be a central engine of the GRB, because it could radiate a large amount of energy $\gtrsim 10^{48} \mathrm{erg}$ via neutrino emission or the Blandford-Znajek mechanism 14] in a short time scale of $\lesssim 2 \mathrm{~s}$ and hence could launch a GRB jet. The merger scenario of the GRB is attractive when a short-hard GRB is associated with a galaxy of low star-formation rate [15, 16] because the collapsar model of GRBs [17] is not preferable. Only numerical relativity can answer quantitatively the question whether the formation of a massive accretion disk is possible in the BH-NS binary merger.

Fully general relativistic study of BH-NS binaries has achieved progress in recent years, both in computations of quasiequilibrium states [18 22] and in dynamical sim- 
ulations of the merger [23 32]. Although these include several simulations of spinning $\mathrm{BH}-\mathrm{NS}$ binaries with a qualitative $\Gamma=2$ ideal-gas EOS, to date, only limited number of simulations have been performed taking into account both the nuclear-theory based EOS and the $\mathrm{BH}$ spin [30]. In particular, we still do not understand the dependence of the merger process and resulting gravitational waveforms on the BH spin and the EOS of the NS in detail. One goal of current numerical relativity is to clarify the effect of the BH spin on the BH-NS binary merger and subsequent NS tidal disruption adopting a wide variety of the NS EOSs.

In this paper, we report our latest results obtained by numerical-relativity simulations with a variety of the NS EOSs and the BH spins. We employ five piecewise polytropic EOSs (see Sec. IIB), all of which do not conflict with the current observation of the $1.97 \pm 0.04 M_{\odot} \mathrm{NS}$. We systematically choose physical parameters such as $\mathrm{BH}$ mass, NS mass, and BH spin in an astrophysically realistic range. While we only consider relatively low mass $\mathrm{BHs}$ in the previous work 31], we adopt a wider range of the $\mathrm{BH}$ mass because a large $\mathrm{BH}$ spin enhances the NS tidal disruption for high mass-ratio binaries. We clarify the dependence on the BH spin and on the NS EOS of the properties of the merger remnants and characteristics of gravitational waves - in particular, the gravitationalwave spectrum.

This paper is organized as follows. In Sec. II, we describe methods for a solution of initial conditions, piecewise polytropic EOSs, and models of BH-NS binaries employed in this paper. In Sec. III] the formulation and methods of numerical simulations are summarized. Section IV presents the numerical results and clarifies the effect of the BH spin and NS EOS on the tidal disruption, merger remnants, and gravitational waves. Section V] is devoted to a summary. Throughout this paper, we adopt the geometrical units in which $G=c=1$, where $G$ and $c$ are the gravitational constant and the speed of light, respectively. Our convention of notation for physically important quantities is summarized in Table [1. The nondimensional spin parameter of the $\mathrm{BH}$, total mass of the system at infinite separation, mass ratio, and compactness of the NS are defined as $a=S_{\mathrm{BH}} / M_{\mathrm{BH}}^{2}$, $m_{0}=M_{\mathrm{BH}}+M_{\mathrm{NS}}, Q=M_{\mathrm{BH}} / M_{\mathrm{NS}}$, and $\mathcal{C}=M_{\mathrm{NS}} / R_{\mathrm{NS}}$, respectively. Latin and Greek indices denote spatial and spacetime components, respectively.

\section{INITIAL CONDITION}

As in our previous works 29, 31], we employ BH-NS binaries in quasiequilibrium states for initial conditions of our numerical simulations. In this section, we summarize the formulation and methods for the computation of a quasiequilibrium state, specifically our method of estimating the spin angular momentum of the $\mathrm{BH}$ in a binary and of determining the position of the rotation axis. The details of the formulation and numerical meth-
TABLE I. Our convention of notation for physically important quantities.

\begin{tabular}{cc}
\hline Symbol & \\
\hline \hline$M_{\mathrm{irr}}$ & The irreducible mass of the $\mathrm{BH}$ \\
$S_{\mathrm{BH}}$ & The magnitude of the $\mathrm{BH}$ spin angular momentum \\
$M_{\mathrm{BH}}$ & The gravitational mass of the $\mathrm{BH}$ in isolation \\
$M_{\mathrm{NS}}$ & The gravitational mass of the NS in isolation \\
$R_{\mathrm{NS}}$ & The circumferential radius of the NS in isolation \\
$M_{0}$ & The Arnowitt-Deser-Misner mass of the system \\
$m_{0}$ & The total mass of the system at infinite separation \\
\hline$Q$ & The mass ratio \\
$\mathcal{C}$ & The compactness of the NS \\
$a$ & The nondimensional spin parameter of the $\mathrm{BH}$ \\
\hline
\end{tabular}

ods, except for the issues on the BH spin, are described in Ref. [22], to which the reader may refer. Computations of the quasiequilibrium states are performed using the spectral-method library LORENE [33].

\section{A. Formulation and methods}

We compute a quasiequilibrium state of the BH-NS binary as a solution of the initial value problem of general relativity 34. As far as the orbital separation is large enough, the time scale of the orbital contraction due to the gravitational radiation reaction is much longer than the orbital period, and, therefore, we may safely neglect the gravitational radiation reaction in the calculation of the quasiequilibrium state. In numerical simulations of the binary coalescences, we have to track $\gtrsim 5$ orbits in order to calculate accurate gravitational waveforms during the late inspiral and merger phases, and hence, the orbital separation of the initial condition has to be large enough. For such initial conditions, we can neglect the gravitational radiation reaction. Thus, we give a $\mathrm{BH}-$ NS binary in a quasicircular orbit, i.e., the binary in an approximate equilibrium state in the corotating frame. To satisfy the quasiequilibrium requirements described above, we assume the existence of a helical Killing vector with the orbital angular velocity $\Omega$,

$$
\xi^{\mu}=\left(\partial_{t}\right)^{\mu}+\Omega\left(\partial_{\varphi}\right)^{\mu} .
$$

We also assume that the NS is in the hydrostatic equilibrium in the corotating frame and has an irrotational velocity field, which is believed to be a reliable approximation to an astrophysically realistic configuration [35, 36.

We compute the three-metric $\gamma_{i j}$, the extrinsic curvature $K_{i j}$, the lapse function $\alpha$, and the shift vector $\beta^{i}$ by a mixture of the extended conformal thin-sandwich approach 37, 38 and the conformal transverse-traceless decomposition [34] in the puncture framework [39 41]. In this formalism, we assume the conformal flatness of the three-metric $\gamma_{i j}=\psi^{4} \hat{\gamma}_{i j}=\psi^{4} f_{i j}$, the stationarity of the conformal three-metric $£_{\xi} \hat{\gamma}_{i j}=0$, the maximal slicing 
condition $K=\gamma^{i j} K_{i j}=0$, and its preservation in time $£_{\xi} K=0$, where $f_{i j}$ is the flat spatial metric. Assuming that the puncture is located at $x_{\mathrm{P}}^{i}$, we set the conformal factor $\psi$ and a weighted lapse function $\Phi \equiv \alpha \psi$ as

$$
\psi=1+\frac{M_{\mathrm{P}}}{2 r_{\mathrm{BH}}}+\phi, \Phi=1-\frac{M_{\Phi}}{2 r_{\mathrm{BH}}}+\eta,
$$

where $M_{\mathrm{P}}$ and $M_{\Phi}$ are positive constants of mass dimension, and $r_{\mathrm{BH}}=\left|x^{i}-x_{\mathrm{P}}^{i}\right|$ is a coordinate distance from the puncture. We adjust $M_{\mathrm{P}}$ to obtain a desired mass of the $\mathrm{BH}, M_{\mathrm{BH}}$, and determine $M_{\Phi}$ so as to satisfy the virial relation, i.e., the equality of the Arnowitt-DeserMisner (ADM) mass and the Komar mass, which holds when the spacetime is stationary and asymptotically flat [42, 43]. $\phi, \beta^{i}$, and $\eta$ are determined by solving elliptic equations derived from the Hamiltonian constraint, momentum constraint, and quasiequilibrium conditions, $£_{\xi} \hat{\gamma}_{i j}=0$ and $£_{\xi} K=0$. We note that these quasiequilibrium conditions can be replaced by $\partial_{t} \hat{\gamma}_{i j}=0$ and $\partial_{t} K=0$ in a conformal flatness approximation. In the puncture framework, we also decompose a conformally weighted extrinsic curvature $\hat{A}_{i j}=\psi^{2} K_{i j}$ as

$$
\hat{A}_{i j}=\hat{\nabla}_{i} W_{j}+\hat{\nabla}_{j} W_{i}-\frac{2}{3} f_{i j} \hat{\nabla}^{k} W_{k}+K_{i j}^{\mathrm{P}},
$$

where $W_{i}$ is an auxiliary three-vector field and $\hat{\nabla}_{i}$ is the covariant derivative associated with $f_{i j} . K_{i j}^{\mathrm{P}}$ is a singular part of the extrinsic curvature, which is associated with the linear and spin angular momenta of the $\mathrm{BH}$ [44],

$$
\begin{aligned}
K_{i j}^{\mathrm{P}} & =\frac{3}{2 r_{\mathrm{BH}}^{2}}\left[l_{i} P_{j}^{\mathrm{BH}}+l_{j} P_{i}^{\mathrm{BH}}-\left(f_{i j}-l_{i} l_{j}\right) l^{k} P_{k}^{\mathrm{BH}}\right] \\
& +\frac{3}{r_{\mathrm{BH}}^{3}}\left[\epsilon_{k i l} S_{\mathrm{P}}^{l} l^{k} l_{j}+\epsilon_{k j l} S_{\mathrm{P}}^{l} l^{k} l_{i}\right],
\end{aligned}
$$

where $l^{i}=x_{\mathrm{BH}}^{i} / r_{\mathrm{BH}}$ is a unit radial vector, $l_{i}=f_{i j} l^{j}$, and $\epsilon_{i j k}$ is the Levi-Civita tensor associated with the flat metric $f_{i j} . P_{i}^{\mathrm{BH}}$ and $S_{\mathrm{P}}^{i}$ are parameters associated with the linear and spin angular momenta of the $\mathrm{BH}$, respectively. Here we determine $P_{i}^{\mathrm{BH}}$ by the condition in which the total linear momentum of the binary vanishes, and we adjust $S_{\mathrm{P}}^{i}$ to obtain a desired spin angular momentum of the $\mathrm{BH}, S_{\mathrm{BH}}^{i}$. The elliptic equation to determine $W_{i}$ is obtained by taking the derivative of Eq. (3) and using the momentum constraint.

The spin angular momentum of the $\mathrm{BH}, S_{\mathrm{BH}}^{i}$, is evaluated on the apparent horizon (hereafter $\mathrm{AH}$ ), $\mathcal{S}$, according to the isolated horizon framework (see Refs. [45, 46] for reviews). Because we do not know the position of the $\mathrm{AH}$ in advance in the puncture framework, we have to determine the location of the AH numerically [47]. On the numerically determined $\mathrm{AH}$, an approximate rotational Killing vector may be defined using the method developed by Cook and Whiting [48] with the normalization condition proposed by Lovelace and his collaborators [49]. We focus only on the case in which the $\mathrm{BH}$ spin is aligned or antialigned with the angular momentum of the binary in this work, and hence, the axis of the $\mathrm{BH}$ spin is uniquely determined. Hereafter we set the rotational axis of the binary, equivalently the axis of the $\mathrm{BH}$ spin, to be the $z$ axis and consider only the approximate Killing vector $\phi^{i}$ associated with the rotation in this direction. Using $\phi^{i}$, we obtain the spin angular momentum of the $\mathrm{BH} S_{\mathrm{BH}}^{(\phi)}=S_{\mathrm{BH}}^{z}$ via the surface integral at the $\mathrm{AH}$,

$$
S_{\mathrm{BH}}^{(\phi)}=\frac{1}{8 \pi} \int_{\mathcal{S}} K_{i j} \phi^{i} d S^{j} .
$$

We adjust $S_{\mathrm{P}}^{z}$ to obtain a desired value of $S_{\mathrm{BH}}^{z}$ (hereafter $\left.S_{\mathrm{BH}}\right)$. We note that $S_{\mathrm{P}}^{i}$ and $S_{\mathrm{BH}}^{i}$ do not agree exactly in the BH-NS binary spacetime due to the contribution to the extrinsic curvature from the NS, associated with $W_{i}$.

Because we adopt a conformal flatness approximation for the induced metric, the Christodoulou mass of the $\mathrm{BH}$ evaluated on the $\mathrm{AH}, M_{\mathcal{H}}=\sqrt{M_{\mathrm{irr}}^{2}+\left(S_{\mathrm{BH}}^{2} / 4 M_{\mathrm{irr}}^{2}\right)}$, and the gravitational mass evaluated at spatial infinity, $M_{\mathrm{BH}}$, do not agree even for a single $\mathrm{BH}$ system due to the presence of so-called junk waves. This difference leads to an ambiguity in defining the nondimensional spin parameter of the $\mathrm{BH}$. Here, we define the nondimensional spin parameter of the $\mathrm{BH}$ with respect to the mass evaluated at spatial infinity, i.e.,

$$
a \equiv \frac{S_{\mathrm{BH}}}{M_{\mathrm{BH}}^{2}} .
$$

The reason for this is that the mass and nondimensional spin parameter of the $\mathrm{BH}$ evaluated at the $\mathrm{AH}$ quickly (in our simulations, within $\sim 1 \mathrm{~ms}$ ) relax to $M_{\mathrm{BH}}$ and $a$, defined at spatial infinity, respectively, as the $\mathrm{BH}$ absorbs the junk radiation in the vicinity of the $\mathrm{BH}[49,50]$. We note that these values show the damping oscillation before the relaxation in the same manner as the "scalarcurvature spin" of Ref. [49] shows, because our method of evaluating these values in the simulation is basically the same as the method to define the scalar-curvature spin in Ref. [49] (see Sec. [IB).

To compute the equations of hydrostatic equilibrium for the NS matter, we assume an ideal fluid, for which the energy-momentum tensor is described by

$$
T^{\mu \nu}=\rho h u^{\mu} u^{\nu}+P g^{\mu \nu},
$$

where $\rho$ is the rest-mass density, $P$ is the pressure, $h \equiv$ $1+\varepsilon+(P / \rho)$ is the specific enthalpy, $\varepsilon$ is the specific internal energy, and $u^{\mu}$ is the four-velocity of the fluid. Basic equations for the hydrostatics are derived from the condition of irrotation, or the vanishing of the vorticity two-form [51, 54,

$$
\omega_{\mu \nu}=\nabla_{\mu}\left(h u_{\nu}\right)-\nabla_{\nu}\left(h u_{\mu}\right)=0,
$$

and the conservation of the specific momentum of the fluid along the helical Killing vector field $£_{\xi}\left(h u_{\mu}\right)=0$. The specific enthalpy is determined from the first integral of the relativistic Euler equation (relativistic Bernoulli integral), and other thermodynamical quantities are subsequently obtained by using the EOS, which is described 
in Sec. IIB. The four-velocity of the fluid is calculated using the velocity potential $\Psi$, as $h u_{i}=\partial_{i} \Psi$ in the assumption of the irrotational velocity field. The elliptic equation for $\Psi$ is derived from the equation of continuity, $\nabla_{\mu}\left(\rho u^{\mu}\right)=0$.

We have no definite condition to determine the location of the center of mass of the binary in the puncture framework and we use this ambiguity to reduce an unphysical initial orbital eccentricity. We found in our previous work [22, 29] that orbits with a small eccentricity could be obtained using the "3PN-J method," i.e., a phenomenological method to determine the location of the rotational axis in which the total angular momentum of the binary for a given value of $\Omega m_{0}$ agrees with that calculated from the third post-Newtonian (3PN) approximation. In the present case in which the $\mathrm{BH}$ has a finite amount of spin angular momentum, we extend the previous 3PN-J method to include the contribution from the $\mathrm{BH}$ spin up to the 2.5PN order [55, 56]. Specifically, the location of the rotational axis is chosen from the condition that the orbital angular momentum of the binary agrees with a sum of nonspin terms given by Eq. (4) of Ref. [57] and of spin terms given by Eq. (7.10) of Ref. [56] for a given value of $\Omega m_{0}$. We show in Sec. IVA that this extended 3PN-J method again leads to the initial condition with a small eccentricity.

\section{B. Piecewise polytropic equations of state}

The matter inside the NS in the late inspiral phase is believed to be well-approximated by a zero-temperature nuclear matter because the cooling time scale of the NS in typical BH-NS binaries is shorter than the time scale of the gravitational radiation reaction [58]. Hence, we employ a cold EOS, for which the rest-mass density, $\rho$, determines all other thermodynamical quantities, for calculating the quasiequilibrium state of the BH-NS binary. To model nuclear-theory-based EOSs at high density with a small number of parameters, we employ a piecewise polytropic EOS. It is a phenomenologically parametrized EOS of the form

$$
P(\rho)=\kappa_{i} \rho^{\Gamma_{i}} \text { for } \rho_{i-1} \leq \rho<\rho_{i} \quad(1 \leq i \leq n),
$$

where $n$ is the number of the pieces used to parametrize an EOS, $\rho_{i}$ is the rest-mass density at the boundary of two neighboring $i$ th and $(i+1)$ th pieces, $\kappa_{i}$ is the polytropic constant for the $i$ th piece, and $\Gamma_{i}$ is the adiabatic index for the $i$ th piece. Here, $\rho_{0}=0, \rho_{n} \rightarrow \infty$, and other parameters $\left(\rho_{i}, \kappa_{i}, \Gamma_{i}\right)$ are freely chosen. Requiring the continuity of the pressure at each $\rho_{i}, 2 n$ free parameters - say $\left(\kappa_{i}, \Gamma_{i}\right)$-determine the EOS completely. The specific internal energy, $\varepsilon$, and hence the specific enthalpy, $h$, are determined by the first law of thermodynamics and continuity of each variable at boundary densities, $\rho_{i}$.

It was shown that piecewise polytropic EOSs with four pieces approximately reproduce most properties of the nuclear-theory-based EOSs at high density [59]. If we focus on low mass NSs with relatively low central density, the EOS at high density plays a minor role. Thus, we adopt a simplified piecewise polytropic EOS composed of two pieces, one of which models the crust EOS and the other of which the core EOS. This simplification is based on the fact that NSs in the observed binary NSs often have fairly small masses $\lesssim 1.4 M_{\odot}$ [60] and the maximum rest-mass density in such NSs may not be so high that the EOS at high density plays only a minor role in determining their structure. Furthermore, the maximum rest-mass density inside the NS should only decrease during the evolution of the BH-NS binary due to tidal elongation of the NS by the companion $\mathrm{BH}$.

Table IIlists the EOSs which we employ in this study. Following Refs. [6, 31], we always fix the EOS for the crust region by the parameters below:

$$
\begin{aligned}
\Gamma_{1} & =1.35692395, \\
\kappa_{1} / c^{2} & =3.99873692 \times 10^{-8}\left(\mathrm{~g} / \mathrm{cm}^{3}\right)^{1-\Gamma_{1}} .
\end{aligned}
$$

The EOS of the core region is determined by two parameters. One is the adiabatic index of the core $\mathrm{EOS}, \Gamma_{2}$. Whereas we find that properties of gravitational waves and the merger remnants depend on $\Gamma_{2}$ in our previous study [31], we always fix $\Gamma_{2}=3$ in this work to focus on clarifying the effect of the $\mathrm{BH}$ spin aside from the difference in the adiabatic index. The other parameter is chosen to be the pressure $p$ at a fiducial density $\rho_{\text {fidu }}=10^{14.7} \mathrm{~g} / \mathrm{cm}^{3}$ because $p$ is closely related to the radius and deformability of the NS 61]. We vary the value of $p$ systematically to investigate the effect of the stiffness of the EOS 62]. With the given values of $\Gamma_{2}$ and $p, \kappa_{2}$ and $\rho_{1}$ are determined as

$$
\begin{aligned}
& \kappa_{2}=p \rho_{\text {fidu }}^{-\Gamma_{2}}, \\
& \rho_{1}=\left(\kappa_{1} / \kappa_{2}\right)^{1 /\left(\Gamma_{2}-\Gamma_{1}\right)} .
\end{aligned}
$$

\section{Models}

Numerical simulations are performed for a wide range of nondimensional $\mathrm{BH}$ spin parameter, $a$, as well as for a variety of the mass ratios, $Q$. For nonspinning BH-NS binaries, we already found that the low mass ratio of $Q \lesssim 3$ is required for tidal disruption of NSs to occur sufficiently outside the innermost stable circular orbit (ISCO) of the $\mathrm{BH}$ unless the EOS is extremely stiff [31]. If the tidal disruption occurs inside or at an orbit very close to the ISCO, we do not see strong effects of the tidal disruption. In such cases, gravitational waveforms are similar to those of a $\mathrm{BH}-\mathrm{BH}$ binary even in the merger phase, and the mass of the remnant disk is negligible 63. However, the allowed range of the mass ratio for the tidal disruption is modified drastically for a $\mathrm{BH}-\mathrm{NS}$ binary with the prograde $\mathrm{BH}$ spin [64, 65] because the ISCO radius 66] of the $\mathrm{BH}$ with a prograde spin becomes smaller by a factor of $1-6$ [67] than that of the nonspinning $\mathrm{BH}$ with 
TABLE II. Key ingredients of the adopted EOSs. $\Gamma_{2}(=3.0)$ is the adiabatic index in the core region and $p$ is the pressure at the fiducial density $\rho_{\mathrm{fidu}}=10^{14.7} \mathrm{~g} / \mathrm{cm}^{3}$, which determines the polytropic constant $\kappa_{2}$ of the core region and $\rho_{1}$, the critical rest-mass density separating the crust and core regions. $M_{\max }$ is the maximum mass of the spherical NS for a given EOS. $R_{135}\left(R_{12}, R_{145}\right)$ and $\mathcal{C}_{135}\left(\mathcal{C}_{12}, \mathcal{C}_{145}\right)$ are the circumferential radius and the compactness of the NS with $M_{\mathrm{NS}}=1.35 M_{\odot}\left(1.2 M_{\odot}, 1.45 M_{\odot}\right)$.

\begin{tabular}{ccc|cccccccc}
\hline Model & $\Gamma_{2}$ & $\log _{10} p\left(\mathrm{~g} / \mathrm{cm}^{3}\right)$ & $\rho_{1}\left(10^{14} \mathrm{~g} / \mathrm{cm}^{3}\right)$ & $M_{\max }\left[M_{\odot}\right]$ & $R_{135}(\mathrm{~km})$ & $\mathcal{C}_{135}$ & $R_{12}(\mathrm{~km})$ & $\mathcal{C}_{12}$ & $R_{145}(\mathrm{~km})$ & $\mathcal{C}_{145}$ \\
\hline \hline $2 \mathrm{H}$ & 3.0 & 13.95 & 0.7033 & 2.835 & 15.23 & 0.1309 & 15.12 & 0.1172 & 15.28 & 0.1401 \\
\hline $1.5 \mathrm{H}$ & 3.0 & 13.75 & 0.9308 & 2.525 & 13.69 & 0.1456 & 13.63 & 0.1300 & 13.72 & 0.1561 \\
\hline $\mathrm{H}$ & 3.0 & 13.55 & 1.232 & 2.249 & 12.27 & 0.1624 & 12.25 & 0.1447 & 12.27 & 0.1744 \\
\hline $\mathrm{HB}$ & 3.0 & 13.45 & 1.417 & 2.122 & 11.61 & 0.1718 & 11.60 & 0.1527 & 11.59 & 0.1848 \\
\hline B & 3.0 & 13.35 & 1.630 & 2.003 & 10.96 & 0.1819 & 10.98 & 0.1614 & 10.93 & 0.1960 \\
\hline
\end{tabular}

the same mass. Strong spin effects for the tidal disruption are also found in the numerical-relativity simulation of the spinning BH-NS binary merger with a simplified, $\Gamma$-law EOS [28]. In this paper we perform a more systematic study of the tidal disruption for different EOSs, masses of each component, and $\mathrm{BH}$ spins.

Table II summarizes several key quantities for the initial conditions in our numerical simulations. The label for the model denotes the EOS name, the mass ratio, the NS mass, and the nondimensional spin parameter of the BH. Specifically, "a75," "a5," and "a-5" correspond to the spin parameters $a=0.75,0.5$, and -0.5 , respectively. For example, HB-Q3M135a5 means that the $\mathrm{EOS}$ is $\mathrm{HB}$ and $\left(Q, M_{\mathrm{NS}}, a\right)=\left(3,1.35 M_{\odot}, 0.5\right)$. Although we vary the NS mass systematically, the results of the merger remnant are reported only for binaries with $M_{\mathrm{NS}}=1.35 M_{\odot}$ in this paper because the difference in the NS mass complicates the properties of the remnant, such as the mass of the disk. Results for $M_{\mathrm{NS}} \neq 1.35 M_{\odot}$ are analyzed only for gravitational waves.

For the same value of the mass ratio, we basically prepare the initial conditions with the same value of the initial angular velocity $\Omega_{0}$ normalized by the total mass of the binary, $\Omega_{0} m_{0}$. For $2 \mathrm{H}$ EOS, in which the NS radius is the largest, we exceptionally adopt a smaller value of $\Omega_{0} m_{0}$ than for other EOSs to guarantee $\gtrsim 5$ orbits before tidal disruption occurs. The reason for this is that the tidal disruption occurs for a large orbital separation in $2 \mathrm{H}$ EOS. When the $\mathrm{BH}$ has a prograde spin, the number of orbits to the merger for a given value of $\Omega_{0} m_{0}$ increases due to spin-orbit repulsive interaction [68], compared to the nonspinning $\mathrm{BH}$ case. On the other hand, when the $\mathrm{BH}$ has a retrograde spin, the number of orbits decreases due to spin-orbit attractive interaction. For $a=-0.5$, the number of orbits is typically by $\sim 1$ orbit smaller than for $a=0$. For this reason, we also prepare the initial condition with a smaller value of $\Omega_{0} m_{0}$ for $\mathrm{H}$ EOS and $a=-0.5$.

\section{METHODS OF SIMULATIONS}

Numerical simulations are performed using an adaptive-mesh refinement (AMR) code SACRA [69]. The formulation, the gauge conditions, the numerical scheme, and the methods of diagnostics are basically the same as those described in Ref. [31], except for the correction in the treatment of hydrodynamic equations in a far region. Thus, we here only briefly review them and describe the present setup of the computational domain for the AMR algorithm and grid resolution.

\section{A. Formulation and numerical methods}

SACRA solves the Einstein evolution equations in the Baumgarte-Shapiro-Shibata-Nakamura (BSSN) formalism [70, 71] with the moving-puncture gauge [39 41]. It evolves a conformal factor $W \equiv \gamma^{-1 / 6}$, the conformal metric $\tilde{\gamma}_{i j} \equiv \gamma^{-1 / 3} \gamma_{i j}$, the trace of the extrinsic curvature $K$, a conformally weighted trace-free part of the extrinsic curvature $\tilde{A}_{i j} \equiv \gamma^{-1 / 3}\left(K_{i j}-K \gamma_{i j}\right)$, and an auxiliary variable $\tilde{\Gamma}^{i} \equiv-\partial_{j} \tilde{\gamma}^{i j}$. Introducing an auxiliary variable $B^{i}$ and a parameter $\eta_{s}$, which we typically set to be $\sim M_{\mathrm{BH}} / M_{\odot}$ in units of $c=G=M_{\odot}=1$, we employ a moving-puncture gauge in the form 72 ]

$$
\begin{aligned}
\left(\partial_{t}-\beta^{j} \partial_{j}\right) \alpha & =-2 \alpha K, \\
\left(\partial_{t}-\beta^{j} \partial_{j}\right) \beta^{i} & =(3 / 4) B^{i}, \\
\left(\partial_{t}-\beta^{j} \partial_{j}\right) B^{i} & =\left(\partial_{t}-\beta^{j} \partial_{j}\right) \tilde{\Gamma}^{i}-\eta_{s} B^{i} .
\end{aligned}
$$

We evaluate the spatial derivative by a fourth-order central finite difference, except for the advection terms, which are evaluated by a fourth-order noncentered, upwind finite difference, and employ a fourth-order RungeKutta method for the time evolution.

To solve the hydrodynamic equations, we evolve $\rho_{*} \equiv$ $\rho \alpha u^{t} W^{-3}, \hat{u}_{i} \equiv h u_{i}$, and $e_{*} \equiv h \alpha u^{t}-P /\left(\rho \alpha u^{t}\right)$. The advection terms are handled with a high-resolution central scheme by Kurganov and Tadmor 73] with a third-order piecewise parabolic interpolation for the cell reconstruction. For the EOS, we decompose the pressure and specific internal energy into cold and thermal parts as

$$
P=P_{\text {cold }}+P_{\text {th }}, \varepsilon=\varepsilon_{\text {cold }}+\varepsilon_{\text {th }} .
$$

We calculate the cold parts of both variables using the piecewise polytropic EOS from the primitive variable $\rho$, and then the thermal part of the specific internal energy is defined from $\varepsilon$ as $\varepsilon_{\text {th }}=\varepsilon-\varepsilon_{\text {cold }}$. Because $\varepsilon_{\text {th }}$ vanishes 
TABLE III. Key parameters and quantities for the initial conditions adopted in numerical simulations. The adopted EOS, mass ratio $(Q)$, NS mass in isolation $\left(M_{\mathrm{NS}}\right)$, nondimensional spin parameter of the $\mathrm{BH}(a)$, initial angular velocity $\left(\Omega_{0}\right)$ in units of $c^{3} / G m_{0}$, baryon rest mass $\left(M_{*}\right)$, compactness of the NS in isolation $(\mathcal{C})$, maximum rest-mass density $\left(\rho_{\max }\right)$, ADM mass of the system $\left(M_{0}\right)$, and total angular momentum of the system $\left(J_{0}\right)$, respectively. See also [31] for models of nonspinning BH-NS binaries.

\begin{tabular}{|c|c|c|c|c|c|c|c|c|c|c|}
\hline Model & EOS & $Q$ & $M_{\mathrm{NS}}\left[M_{\odot}\right]$ & $a$ & $G \Omega_{0} m_{0} / c^{3}$ & $M_{*}\left[M_{\odot}\right]$ & $\mathcal{C}$ & $\rho_{\max }\left(10^{14} \mathrm{~g} / \mathrm{cm}^{3}\right)$ & $M_{0}\left[M_{\odot}\right]$ & $J_{0}\left[G M_{\odot}^{2} / c\right]$ \\
\hline 2H-Q2M135a75 & $2 \mathrm{H}$ & 2 & 1.35 & 0.75 & 0.025 & 1.455 & 0.1309 & 3.740 & 4.014 & 13.83 \\
\hline $1.5 \mathrm{H}-\mathrm{Q} 2 \mathrm{M} 135 \mathrm{a} 75$ & $1.5 \mathrm{H}$ & 2 & 1.35 & 0.75 & 0.028 & 1.468 & 0.1456 & 5.104 & 4.012 & 13.42 \\
\hline H-Q2M135a75 & $\mathrm{H}$ & 2 & 1.35 & 0.75 & 0.028 & 1.484 & 0.1624 & 7.019 & 4.012 & 13.42 \\
\hline HB-Q2M135a75 & HB & 2 & 1.35 & 0.75 & 0.028 & 1.493 & 0.1718 & 8.263 & 4.012 & 13.42 \\
\hline B-Q2M135a75 & B & 2 & 1.35 & 0.75 & 0.028 & 1.503 & 0.1819 & 9.762 & 4.012 & 13.42 \\
\hline 2H-Q2M135a5 & $2 \mathrm{H}$ & 2 & 1.35 & 0.5 & 0.025 & 1.455 & 0.1309 & 3.740 & 4.014 & 14.02 \\
\hline 1.5H-Q2M135a5 & $1.5 \mathrm{H}$ & 2 & 1.35 & 0.5 & 0.028 & 1.468 & 0.1456 & 5.104 & 4.012 & 13.63 \\
\hline H-Q2M135a5 & $\mathrm{H}$ & 2 & 1.35 & 0.5 & 0.028 & 1.484 & 0.1624 & 7.018 & 4.012 & 13.63 \\
\hline HB-Q2M135a5 & $\mathrm{HB}$ & 2 & 1.35 & 0.5 & 0.028 & 1.493 & 0.1718 & 8.263 & 4.012 & 13.63 \\
\hline B-Q2M135a5 & $\mathrm{B}$ & 2 & 1.35 & 0.5 & 0.028 & 1.503 & 0.1819 & 9.762 & 4.012 & 13.63 \\
\hline 2H-Q2M135a-5 & $2 \mathrm{H}$ & 2 & 1.35 & -0.5 & 0.022 & 1.455 & 0.1309 & 3.740 & 4.019 & 15.15 \\
\hline H-Q2M135a-5 & $\mathrm{H}$ & 2 & 1.35 & -0.5 & 0.025 & 1.484 & 0.1624 & 7.018 & 4.017 & 14.74 \\
\hline HB-Q2M135a-5 & $\mathrm{HB}$ & 2 & 1.35 & -0.5 & 0.028 & 1.493 & 0.1718 & 8.262 & 4.015 & 14.41 \\
\hline B-Q2M135a-5 & $\mathrm{B}$ & 2 & 1.35 & -0.5 & 0.028 & 1.503 & 0.1819 & 9.760 & 4.015 & 14.41 \\
\hline 2H-Q2M12a75 & $2 \mathrm{H}$ & 2 & 1.2 & 0.75 & 0.025 & 1.282 & 0.1172 & 3.465 & 3.568 & 10.93 \\
\hline H-Q2M12a75 & $\mathrm{H}$ & 2 & 1.2 & 0.75 & 0.028 & 1.303 & 0.1447 & 6.421 & 3.566 & 10.60 \\
\hline HB-Q2M12a75 & $\mathrm{HB}$ & 2 & 1.2 & 0.75 & 0.028 & 1.310 & 0.1527 & 7.523 & 3.566 & 10.60 \\
\hline B-Q2M12a75 & $\mathrm{B}$ & 2 & 1.2 & 0.75 & 0.028 & 1.317 & 0.1614 & 8.833 & 3.566 & 10.60 \\
\hline 2H-Q2M145a75 & $2 \mathrm{H}$ & 2 & 1.45 & 0.75 & 0.025 & 1.572 & 0.1401 & 3.926 & 4.312 & 15.96 \\
\hline H-Q2M145a75 & $\mathrm{H}$ & 2 & 1.45 & 0.75 & 0.028 & 1.607 & 0.1744 & 7.452 & 4.309 & 15.48 \\
\hline HB-Q2M145a75 & $\mathrm{HB}$ & 2 & 1.45 & 0.75 & 0.028 & 1.617 & 0.1848 & 8.811 & 4.309 & 15.48 \\
\hline B-Q2M145a75 & $\mathrm{B}$ & 2 & 1.45 & 0.75 & 0.028 & 1.629 & 0.1960 & 10.46 & 4.309 & 15.48 \\
\hline 2H-Q3M135a75 & $2 \mathrm{H}$ & 3 & 1.35 & 0.75 & 0.028 & 1.455 & 0.1309 & 3.737 & 5.357 & 20.00 \\
\hline 1.5H-Q3M135a75 & $1.5 \mathrm{H}$ & 3 & 1.35 & 0.75 & 0.030 & 1.468 & 0.1456 & 5.100 & 5.355 & 19.64 \\
\hline H-Q3M135a75 & $\mathrm{H}$ & 3 & 1.35 & 0.75 & 0.030 & 1.484 & 0.1624 & 7.013 & 5.355 & 19.64 \\
\hline HB-Q3M135a75 & $\mathrm{HB}$ & 3 & 1.35 & 0.75 & 0.030 & 1.493 & 0.1718 & 8.256 & 5.355 & 19.64 \\
\hline B-Q3M135a75 & $\mathrm{B}$ & 3 & 1.35 & 0.75 & 0.030 & 1.503 & 0.1819 & 9.753 & 5.355 & 19.63 \\
\hline 2H-Q3M135a5 & $2 \mathrm{H}$ & 3 & 1.35 & 0.5 & 0.028 & 1.455 & 0.1309 & 3.737 & 5.357 & 20.36 \\
\hline 1.5H-Q3M135a5 & $1.5 \mathrm{H}$ & 3 & 1.35 & 0.5 & 0.030 & 1.468 & 0.1456 & 5.100 & 5.356 & 20.02 \\
\hline H-Q3M135a5 & $\mathrm{H}$ & 3 & 1.35 & 0.5 & 0.030 & 1.484 & 0.1624 & 7.012 & 5.356 & 20.01 \\
\hline HB-Q3M135a5 & HB & 3 & 1.35 & 0.5 & 0.030 & 1.493 & 0.1718 & 8.255 & 5.356 & 20.01 \\
\hline B-Q3M135a5 & B & 3 & 1.35 & 0.5 & 0.030 & 1.503 & 0.1819 & 9.753 & 5.356 & 20.01 \\
\hline HB-Q3M135a-5 & $\mathrm{HB}$ & 3 & 1.35 & -0.5 & 0.030 & 1.493 & 0.1718 & 8.253 & 5.359 & 21.46 \\
\hline 2H-Q3M145a75 & $2 \mathrm{H}$ & 3 & 1.45 & 0.75 & 0.028 & 1.572 & 0.1401 & 3.923 & 5.754 & 23.07 \\
\hline H-Q3M145a75 & $\mathrm{H}$ & 3 & 1.45 & 0.75 & 0.030 & 1.607 & 0.1744 & 7.445 & 5.751 & 22.65 \\
\hline HB-Q3M145a75 & $\mathrm{HB}$ & 3 & 1.45 & 0.75 & 0.030 & 1.617 & 0.1848 & 8.803 & 5.751 & 22.65 \\
\hline B-Q3M145a75 & $\mathrm{B}$ & 3 & 1.45 & 0.75 & 0.030 & 1.629 & 0.1960 & 10.45 & 5.751 & 22.65 \\
\hline 2H-Q4M135a75 & $2 \mathrm{H}$ & 4 & 1.35 & 0.75 & 0.030 & 1.455 & 0.1309 & 3.735 & 6.702 & 26.07 \\
\hline H-Q4M135a75 & $\mathrm{H}$ & 4 & 1.35 & 0.75 & 0.032 & 1.484 & 0.1624 & 7.007 & 6.700 & 25.62 \\
\hline HB-Q4M135a75 & $\mathrm{HB}$ & 4 & 1.35 & 0.75 & 0.032 & 1.493 & 0.1718 & 8.249 & 6.700 & 25.63 \\
\hline B-Q4M135a75 & B & 4 & 1.35 & 0.75 & 0.032 & 1.503 & 0.1819 & 9.746 & 6.700 & 25.62 \\
\hline 2H-Q4M135a5 & $2 \mathrm{H}$ & 4 & 1.35 & 0.5 & 0.035 & 1.455 & 0.1309 & 3.732 & 6.698 & 25.64 \\
\hline H-Q4M135a5 & $\mathrm{H}$ & 4 & 1.35 & 0.5 & 0.035 & 1.484 & 0.1624 & 7.004 & 6.698 & 25.63 \\
\hline HB-Q4M135a5 & $\mathrm{HB}$ & 4 & 1.35 & 0.5 & 0.035 & 1.493 & 0.1718 & 8.244 & 6.698 & 25.63 \\
\hline B-Q4M135a5 & B & 4 & 1.35 & 0.5 & 0.035 & 1.503 & 0.1819 & 9.740 & 6.698 & 25.63 \\
\hline 2H-Q5M135a75 & $2 \mathrm{H}$ & 5 & 1.35 & 0.75 & 0.036 & 1.455 & 0.1309 & 3.730 & 8.044 & 30.95 \\
\hline H-Q5M135a75 & $\mathrm{H}$ & 5 & 1.35 & 0.75 & 0.036 & 1.484 & 0.1624 & 7.000 & 8.044 & 30.95 \\
\hline HB-Q5M135a75 & HB & 5 & 1.35 & 0.75 & 0.036 & 1.493 & 0.1718 & 8.241 & 8.044 & 30.95 \\
\hline B-Q5M135a75 & $\mathrm{B}$ & 5 & 1.35 & 0.75 & 0.036 & 1.503 & 0.1819 & 9.736 & 8.043 & 30.95 \\
\hline
\end{tabular}


in the absence of shock heating, $\varepsilon_{\text {th }}$ is regarded as the finite-temperature part. In this paper, we adopt a $\Gamma$-law ideal-gas EOS for the thermal part,

$$
P_{\mathrm{th}}=\left(\Gamma_{\mathrm{th}}-1\right) \rho \varepsilon_{\mathrm{th}},
$$

to determine the thermal part of the pressure, and choose $\Gamma_{\text {th }}$ equal to the adiabatic index in the crust region, $\Gamma_{1}$, for simplicity.

Because the vacuum is not allowed in any conservative hydrodynamic scheme, we put an artificial atmosphere of a small density outside the NS in the same way as done in our previous work [31]. The total rest mass of the atmosphere is always less than $10^{-4} M_{\odot}$, and hence, we can safely neglect spurious effects by accretion of the atmosphere onto the remnant disk as long as the disk mass is much larger than $10^{-4} M_{\odot}$.

\section{B. Diagnostics}

We extract gravitational waves by calculating the outgoing part of the Weyl scalar $\Psi_{4}$ at finite coordinate radii $r=400-800 M_{\odot}$ and by integrating $\Psi_{4}$ twice in time as

$$
h_{+}(t)-i h_{\times}(t)=-\int^{t} d t^{\prime} \int^{t^{\prime}} d t^{\prime \prime} \Psi_{4}\left(t^{\prime \prime}\right) .
$$

In our previous works [29, 31], we directly perform this integration of $\Psi_{4}(t)$ and then subtract a quadratic function of the form $a_{2} t^{2}+a_{1} t+a_{0}$ to eliminate unphysical drift components in the waveform, using the leastsquare fitting to obtain constants $a_{0}, a_{1}$, and $a_{2}$. In this work, we adopt a "fixed-frequency integration" method proposed by Reisswig and Pollney [74] to obtain gravitational waveforms with less unphysical components. In this method, we first perform a Fourier transformation of $\Psi_{4}$ as

$$
\tilde{\Psi}_{4}(\omega)=\int d t \Psi_{4}(t) e^{i \omega t}
$$

Using this, Eq. (19) is rewritten as

$$
h_{+}(t)-i h_{\times}(t)=\frac{1}{2 \pi} \int \frac{\tilde{\Psi}_{4}(\omega)}{\omega^{2}} e^{-i \omega t} d \omega .
$$

We then replace $1 / \omega^{2}$ of the integrand with $1 / \omega_{0}^{2}$ for $|\omega|<\omega_{0}$, where $\omega_{0}$ is a positive free parameter in this method. By appropriately choosing $\omega_{0}$, this procedure suppresses unphysical, low-frequency components of gravitational waves. As proposed in Ref. [74], we choose $\omega_{0}$ to be $\sim 0.8 m \Omega_{0}$ for $m \neq 0$ mode gravitational waves, where $m$ is the azimuthal quantum number. For the $m=0$ mode gravitational waves, we adopt $\omega_{0} \sim 0.8 \Omega_{0}$ and confirm that our results depend only very weakly on this choice. We also adopt this method to calculate the energy $\Delta E$ and angular momentum $\Delta J$ radiated by gravitational waves. Exceptionally, we adopt the previous method of direct time integration to estimate the orbital eccentricity in the inspiral phase because the fixedfrequency integration method may change the modulation in the gravitational waveform.

For comparisons between numerically calculated gravitational waveforms and those calculated in the PN approximations, we use the Taylor-T4 formula for two-point masses in circular orbits [75] with an additional contribution from the BH spin angular momentum 76]. In this formula, the time evolution of the orbital angular velocity $\Omega(t)$ and orbital phase $\Theta(t)$ are computed using a nondimensional angular velocity $X(t) \equiv\left[m_{0} \Omega(t)\right]^{2 / 3}$ by

$$
\begin{aligned}
\frac{d X}{d t}= & \frac{64 \nu X^{5}}{5 m_{0}}\left[1-\left(\frac{743}{336}+\frac{11}{4} \nu\right) X+\left(4 \pi-\frac{113}{12} \chi+\frac{19}{6} \nu a\right) X^{3 / 2}\right. \\
& +\left(\frac{34103}{18144}+5 \chi^{2}+\frac{13661}{2016} \nu+\frac{59}{18} \nu^{2}\right) X^{2} \\
& -\left\{\left(\frac{4159}{672}+\frac{189}{8} \nu\right) \pi+\left(\frac{31571}{1008}-\frac{1165}{24} \nu\right) \chi+\frac{3}{4} \chi^{3}-\left(\frac{21863}{1008} \nu-\frac{79}{6} \nu^{2}\right) a\right\} X^{5 / 2} \\
& +\left\{\frac{16447322263}{139708800}-\frac{1712}{105} \gamma_{E}+\frac{16}{3} \pi^{2}-\left(\frac{56198689}{217728}-\frac{451}{48} \pi^{2}\right) \nu+\frac{541}{896} \nu^{2}-\frac{5605}{2592} \nu^{3}\right. \\
& \left.-\frac{856}{105} \ln (16 X)-\frac{80 \pi}{3} \chi+\left(\frac{64153}{1008}-\frac{457}{36} \nu\right) \chi^{2}+\left(\frac{20}{3} \pi-\frac{1135}{36} \chi\right) \nu a\right\} X^{3} \\
& -\left\{\left(\frac{4415}{4032}-\frac{358675}{6048} \nu-\frac{91495}{1512} \nu^{2}\right) \pi+\left(\frac{2529407}{27216}-\frac{845827}{6048} \nu+\frac{41551}{864} \nu^{2}\right) \chi-12 \pi \chi^{2}\right. \\
& \left.\left.+\left(\frac{1505}{24}+\frac{\nu}{8}\right) \chi^{3}-\left(\frac{1580239}{54432}-\frac{451597}{6048} \nu^{2}+\frac{2045}{432} \nu^{3}+\frac{107}{6} \nu \chi^{2}\right) a\right\} X^{7 / 2}\right]
\end{aligned}
$$

$$
\frac{d \Theta}{d t}=\frac{X^{3 / 2}}{m_{0}}
$$

where $\nu \equiv Q /(1+Q)^{2}, \chi=a Q /(1+Q)$, and $\gamma_{E} \approx 0.5772$ 
is the Euler constant. After $X(t)$ and $\Theta(t)$ are obtained, we calculate the complex gravitational-wave amplitude $h^{22}$ of the $(l, m)=(2,2)$ mode and the spectrum up to the $3 \mathrm{PN}$ order using the formula shown in Refs. [76, 77]. Here, $h^{22}$ is

$$
\begin{aligned}
h^{22}= & -8 \sqrt{\frac{\pi}{5}} \frac{\nu m_{0}}{D} e^{-2 i \Theta} X\left[1-\left(\frac{107}{42}-\frac{55}{42} \nu\right) X+\left(2 \pi-\frac{4}{3} \chi+\frac{2}{3} \nu a\right) X^{3 / 2}\right. \\
& -\left(\frac{2173}{1512}+\frac{1069}{216} \nu-\frac{2047}{1512} \nu^{2}\right) X^{2}-\left\{\left(\frac{107}{21}-\frac{34}{21} \nu\right) \pi+24 i \nu\right\} X^{5 / 2} \\
& +\left\{\frac{27027409}{646800}-\frac{856}{105} \gamma_{E}+\frac{2}{3} \pi^{2}-\frac{428}{105} \ln (16 X)-\left(\frac{278185}{33264}-\frac{41}{96} \pi^{2}\right) \nu-\frac{20261}{2772} \nu^{2}\right. \\
& \left.\left.+\frac{114635}{99792} \nu^{3}+\frac{428}{105} i \pi\right\} X^{3}\right],
\end{aligned}
$$

where $D$ is the distance between the center of mass of the binary and an observer. Hereafter, we simply refer to this formula as the Taylor-T4 formula irrespective of the presence of the $\mathrm{BH}$ spin. Another way for deriving an approximate waveform is to employ an effective onebody approach (see Ref. 78] and references therein for reviews). In accompanied papers [63], comparisons between numerical waveforms and those of the effective one body approach are extensively performed.

To estimate the mass of the remnant disk, we calculate the total rest mass outside the $\mathrm{AH}$

$$
M_{r>r_{\mathrm{AH}}} \equiv \int_{r>r_{\mathrm{AH}}} \rho_{*} d^{3} x,
$$

where $r_{\mathrm{AH}}=r_{\mathrm{AH}}(\theta, \varphi)$ is the radius of the $\mathrm{AH}$ as a function of the angular coordinates. We note that we systematically underestimated disk masses in our previous works performed with an old version of SACRA [29, 31], because we evolved hydrodynamic variables and estimated disk masses only in the finer domains (described in Sec. IIIC) of the size $\sim 200^{3} \mathrm{~km}^{3}$. Such a domain size is insufficient for the estimation of the disk mass if tidal disruption occurs at a distant orbit, especially for the case in which the NS radius is large $(\sim 15 \mathrm{~km})$. In this study, we correct the treatment of hydrodynamics and the estimation of disk masses: We follow the hydrodynamics for a wide computational domain of the size $1000^{3}-2000^{3} \mathrm{~km}^{3}$. We still possibly underestimated disk masses because some of the material escapes from our computational domains and we cannot follow their return which would occur if they are bounded.

We determine key quantities of the remnant $\mathrm{BH}$, i.e., the mass $M_{\mathrm{BH}, \mathrm{f}}$ and nondimensional spin parameter $a_{\mathrm{f}}$, from the circumferential radius of the $\mathrm{AH}$, assuming that the deviation from the Kerr spacetime is negligible in the vicinity of a $\mathrm{BH}$ horizon. We estimate the remnant $\mathrm{BH}$ mass, $M_{\mathrm{BH}, \mathrm{f}}$, from the circumferential radius of the $\mathrm{AH}$ along the equatorial plane $C_{e}$ divided by $4 \pi$, i.e., $C_{e} / 4 \pi$, which gives the $\mathrm{BH}$ mass in the stationary vacuum $\mathrm{BH}$ spacetime. Similarly, the nondimensional spin parameter of the remnant $\mathrm{BH}, a_{\mathrm{f}}$, is estimated from the ratio of the circumferential radius of the $\mathrm{AH}$ along the meridional plane $C_{p}$ to $C_{e}$ using the relation

$$
\frac{C_{p}}{C_{e}}=\frac{\sqrt{2 \hat{r}_{+}}}{\pi} E\left(\frac{a_{\mathrm{f}}^{2}}{2 \hat{r}_{+}}\right) .
$$

This also holds for the stationary vacuum $\mathrm{BH}$ with the nondimensional spin parameter $a_{\mathrm{f}}$. Here, $\hat{r}_{+}=1+$ $\sqrt{1-a_{\mathrm{f}}^{2}}$ is a normalized radius of the horizon, and $E(z)$ is an elliptic integral

$$
E(z)=\int_{0}^{\pi / 2} \sqrt{1-z \sin ^{2} \theta} d \theta .
$$

For comparison, the nondimensional spin parameter of the remnant $\mathrm{BH}$ is also estimated from $C_{e}$ and the irreducible mass of the remnant $\mathrm{BH} M_{\mathrm{irr}, \mathrm{f}}$ using the relation

$$
M_{\mathrm{irr}, \mathrm{f}}=\frac{C_{e}}{4 \sqrt{2} \pi} \sqrt{1+\sqrt{1-a_{\mathrm{f}}^{2}}},
$$

which holds for the stationary vacuum BH. The spin parameter obtained using this relation is referred to as $a_{\mathrm{f} 2}$ according to Ref. 29]. Finally, we also estimate $a_{\mathrm{f}}$ from the values of the remnant $\mathrm{BH}$ computed using approximate conservation laws

$$
\begin{aligned}
M_{\mathrm{BH}, \mathrm{c}} & \equiv M_{0}-M_{r>r_{\mathrm{AH}}}-\Delta E, \\
J_{\mathrm{BH}, \mathrm{c}} & \equiv J_{0}-J_{r>r_{\mathrm{AH}}}-\Delta J,
\end{aligned}
$$

where the total angular momentum of the material located outside the AH, $J_{r>r_{\mathrm{AH}}}$, is approximately defined by

$$
J_{r>r_{\mathrm{AH}}} \equiv \int_{r>r_{\mathrm{AH}}} \rho_{*} h u_{\varphi} d^{3} x .
$$

Here, we assume that the orbital angular momentum of the $\mathrm{BH}$ is negligible. The nondimensional spin parameter of the remnant $\mathrm{BH}$ is defined by $a_{\mathrm{f} 1} \equiv J_{\mathrm{BH}, \mathrm{c}} / M_{\mathrm{BH}, \mathrm{c}}^{2}$, again according to Ref. [29]. 


\section{Setup of AMR grids}

In SACRA, an AMR algorithm is implemented so that both the radii of compact objects in the near zone and the characteristic gravitational wavelengths in the wave zone can be covered with sufficient grid resolutions simultaneously. Our AMR grids consist of a number of computational domains, each of which has the uniform, vertexcentered Cartesian grids with $(2 N+1,2 N+1, N+1)$ grid points for $(x, y, z)$ with the equatorial plane symmetry at $z=0$. We always choose $N=50$ for the best resolved runs in this work. We also perform simulations with $N=36$ and 42 for several arbitrary chosen models to check the convergence of the results and find approximately the same level of convergence as that found in the previous work (see the Appendix of Ref. 31]). In the Appendix of this paper, we show the convergence of gravitational waveforms and the masses of the remnant disks. The AMR grids are classified into two categories: one is a coarser domain, which covers a wide region, including both the $\mathrm{BH}$ and NS, with its origin fixed at the approximate center of mass throughout the simulation. The other is a finer domain, two sets of which comove with compact objects and cover the region in the vicinity of these objects. We denote the edge length of the largest domain, the number of the coarser domains, and the number of the finer domains by $2 L, l_{c}$, and $2 l_{f}$, respectively. Namely, the total number of the domains is $l_{c}+2 l_{f}$. The grid spacing for each domain is $h_{l}=L /\left(2^{l} N\right)$, where $l=0-\left(l_{c}+l_{f}-1\right)$ is the depth of each domain.

Table IV summarizes the parameters of the grid structure for our simulations. The structure of the AMR grids depends primarily on the mass ratio of the binary because the distances between two objects and the center of mass depend strongly on the mass ratio for our initial models. Specifically, we choose $\left(l_{c}, l_{f}\right)=(4,4)$ for all binaries with $M_{\mathrm{NS}}=1.35 M_{\odot}$ and $Q=2,3$, and 4 . We choose $\left(l_{c}, l_{f}\right)=(3,5)$ for binaries with $Q=5$. For binaries with $M_{\mathrm{NS}} \neq 1.35 M_{\odot}$, we choose $\left(l_{c}, l_{f}\right)=(3,4)$ because we do not evaluate disk masses for them. In all the simulations, $L$ is chosen to be larger than or comparable to the gravitational wavelengths at an initial instant $\lambda_{0} \equiv \pi / \Omega_{0}$. One of the two finest regions covers the semimajor axis of the NS by $\sim 42-45$ grid points. The other covers the coordinate radius of the $\mathrm{AH}$ typically by $\sim 20$ grid points, depending on the mass ratio and the $\mathrm{BH}$ spin. For the $Q=5$ runs, the total memory required is about $11 \mathrm{G}$ bytes. We perform numerical simulations with personal computers of $12 \mathrm{G}$ bytes memory and of core-i7X processors with clock speeds of 3.2 or $3.33 \mathrm{GHz}$. We use 2-6 processors to perform one job with an OPEN-MP library. The typical computational time required to perform one simulation (for $\sim 50 \mathrm{~ms}$ in physical time of coalescence for the $a=0.75$ case) is 4 weeks for the 6 processor case.

\section{NUMERICAL RESULTS}

We present numerical results of our simulations, focusing, in particular, on their dependence on the $\mathrm{BH}$ spin and NS EOS. First, we review general merger processes in Sec. IVA Sections IVB IVC, and IVD are devoted to the analysis of properties of the remnant disk and $\mathrm{BH}$ formed after the merger. Gravitational waveforms are shown in Sec. IVE, their spectra in Sec. IVF, and the energy and angular momentum radiated by gravitational waves in Sec. IV G.

\section{A. Overview of the merger process}

Figure 1 plots the evolution of the coordinate separation defined by $x_{\mathrm{sep}}^{i}=x_{\mathrm{NS}}^{i}-x_{\mathrm{BH}}^{i}$ for models HBQ2M135a5, HB-Q2M135, and HB-Q2M135a-5, for which $\Omega_{0} m_{0}$ takes the same values. Here, $x_{\mathrm{NS}}^{i}$ is the position of the maximum rest-mass density and $x_{\mathrm{BH}}^{i}$ is the location of the puncture, $x_{\mathrm{P}}^{i}$. Figure 1 shows that the numbers of orbits increases as the $\mathrm{BH}$ spin increases from retrograde to prograde [28]. Specifically, the number of orbit are $\sim 7,5.5$, and 4 for $a=0.5,0$, and -0.5 , respectively. This difference comes primarily from the spin-orbit interaction between these two angular momenta [79]; in the PN approximation, a force proportional to the inner product of the orbital and spin angular momenta of two objects appears at $1.5 \mathrm{PN}$ order. Here, we do not have to consider the NS spin angular momentum in the assumption of the irrotational velocity field and, therefore, we only consider the interaction between the orbital and $\mathrm{BH}$ spin angular momenta throughout this paper. When these two angular momenta are parallel and the inner product is positive $(a>0)$, an additional repulsive force works between the BH and NS. This repulsive force reduces the orbital angular velocity because the centrifugal force associated with the orbital motion can be reduced, and hence, the luminosity of gravitational radiation, which is proportional to $\Omega^{10 / 3}$, is also reduced. This strong dependence of the luminosity on $\Omega$ makes the approaching velocity smaller in the late inspiral phase, and, therefore, the number of orbits increases. Conversely, when these two angular momenta are antiparallel $(a<0)$, an additional attractive force increases the angular velocity and gravitational-wave luminosity in the late inspiral phase. In this case, the orbital separation decreases faster due to a larger approaching velocity, and the number of orbits becomes smaller as the retrograde $\mathrm{BH}$ spin increases. All these results agree qualitatively with those of Ref. 28].

The fate of BH-NS binaries is classified into two categories. One is the case in which the NS is disrupted by the $\mathrm{BH}$ tidal field before the $\mathrm{BH}$ swallows the NS, and the other is the case in which the BH swallows the NS without tidal disruption. In this paper, we focus mainly on the former case. We plot snapshots of the rest-mass density profiles and the location of the $\mathrm{AH}$ on the equatorial 
TABLE IV. Setup of the grid structure for the simulation with our AMR algorithm. $l_{c}$ and $l_{f}$ are the number of coarser domains and a half of finer domains, respectively. $\Delta x=h_{l}=L /\left(2^{l} N\right)\left(l=l_{c}+l_{f}-1\right)$ is the grid spacing at the finest-resolution domain with $L$ being the location of the outer boundaries along each axis. $R_{\text {diam }} / \Delta x$ denotes the grid number assigned inside the semimajor diameter of the NS. $\lambda_{0}$ is the gravitational wavelength of the initial configuration. (See Ref. [31] for models with nonspinning BHs.)

\begin{tabular}{|c|c|c|c|c|}
\hline Model & $l_{c} l_{f}$ & $\Delta x / M_{0}$ & $R_{\text {diam }} / \Delta x$ & $L / \lambda_{0}$ \\
\hline 2H-Q2 & 44 & 0.0471 & 90.8 & .386 \\
\hline $1.5 \mathrm{H}-\mathrm{Q}$ & 44 & 26 & 7.7 & 417 \\
\hline $\mathrm{H}-\mathrm{Q}$ & 44 & & .2 & 138 \\
\hline HB-Q & 44 & 47 & .1 & 968 \\
\hline B-Q2 & 44 & 24 & 6.7 & 837 \\
\hline $2 \mathrm{H}-\mathrm{Q}$ & 44 & 0 & .8 & 378 \\
\hline $1.5 \mathrm{H}-\mathrm{C}$ & 44 & & 7.7 & 410 \\
\hline $\mathrm{H}-\mathrm{Q}$ & 44 & 0 & 6.2 & 131 \\
\hline $\mathrm{HB}-\mathrm{Q}$ & 44 & 0 & 7.2 & 962 \\
\hline B-Q2 & 44 & 0 . & 6.7 & 831 \\
\hline $2 \mathrm{H}-\mathrm{C}$ & 44 & 0 & o. & 092 \\
\hline $\mathrm{H}-\mathrm{Q}$ & 44 & & .1 & 902 \\
\hline HB-Q2 & 44 & & .1 & 962 \\
\hline B-Q2M1: & 44 & 24 & 6.7 & .831 \\
\hline 2$]$ & 34 & & & 176 \\
\hline H-Q2 & 34 & & & 252 \\
\hline HB-Q & 34 & & .7 & 162 \\
\hline B-Q2 & 34 & 0 & 4.2 & .102 \\
\hline $2 \mathrm{H}-\mathrm{C}$ & 34 & & & 166 \\
\hline H-Q2 & 34 & & & 985 \\
\hline HB-Q & 34 & & & 896 \\
\hline B-Q2] & 34 & & .1 & 829 \\
\hline 75 & 44 & & 85.5 & 84 \\
\hline $1.5 \mathrm{H}-$ & 44 & & 0 & 986 \\
\hline $\mathrm{H}-\mathrm{C}$ & 44 & & 7 & 718 \\
\hline HB- & 44 & & .6 & 581 \\
\hline B-Q3I & 44 & & .9 & .431 \\
\hline $2 \mathrm{H}-$ & 44 & & 9 & 997 \\
\hline $1.5 \mathrm{H}$ & 44 & & 4.0 & 980 \\
\hline $\mathrm{H}-\mathrm{C}$ & 44 & & 4.7 & 712 \\
\hline HB-Q & 44 & & .7 & 576 \\
\hline 3-Q3 & 44 & 43 & 85.3 & 1.471 \\
\hline HB-Q3 & 44 & 0 . & 85.7 & 1.576 \\
\hline $2 \mathrm{H}-\mathrm{Q} 3$ & 34 & 28 & .7 & .933 \\
\hline H-Q3I & 34 & 0. & 37.4 & .760 \\
\hline HB-Q3 & 34 & 34 & 86.6 & 0.712 \\
\hline B-Q3M145a75 & 34 & 0.0214 & 87.7 & 0.651 \\
\hline $2 \mathrm{H}-\mathrm{Q} 4$ & 44 & 96 & 83.4 & .804 \\
\hline $\mathrm{H}-\mathrm{Q} 4 \mathrm{~N}$ & 44 & 23 & 84.5 & 1.450 \\
\hline HB-Q4M1: & 44 & 0.0203 & 86.5 & 1.319 \\
\hline B-Q4M135a75 & 44 & 0.0190 & 85.8 & 1.237 \\
\hline $2 \mathrm{H}-\mathrm{Q}$ & 44 & 0 & 83.2 & 2.097 \\
\hline $\mathrm{H}-\mathrm{Q} 4 \mathrm{~N}$ & 44 & 19 & 9 & 1.548 \\
\hline HB-Q4M135a5 & 44 & 0.0205 & 85.5 & 1.448 \\
\hline B-Q4M1: & 4 & 38 & 86.4 & 1.332 \\
\hline $2 \mathrm{H}-$ & 3 & 0 & .3 & .718 \\
\hline H-Q5M135a75 & 35 & 0 & 86.2 & 1.314 \\
\hline HB-Q5M135a75 & 3 & 67 & 86.3 & 1.224 \\
\hline B-Q5M135a75 & 35 & 0.0159 & 84.6 & 1.159 \\
\hline
\end{tabular}



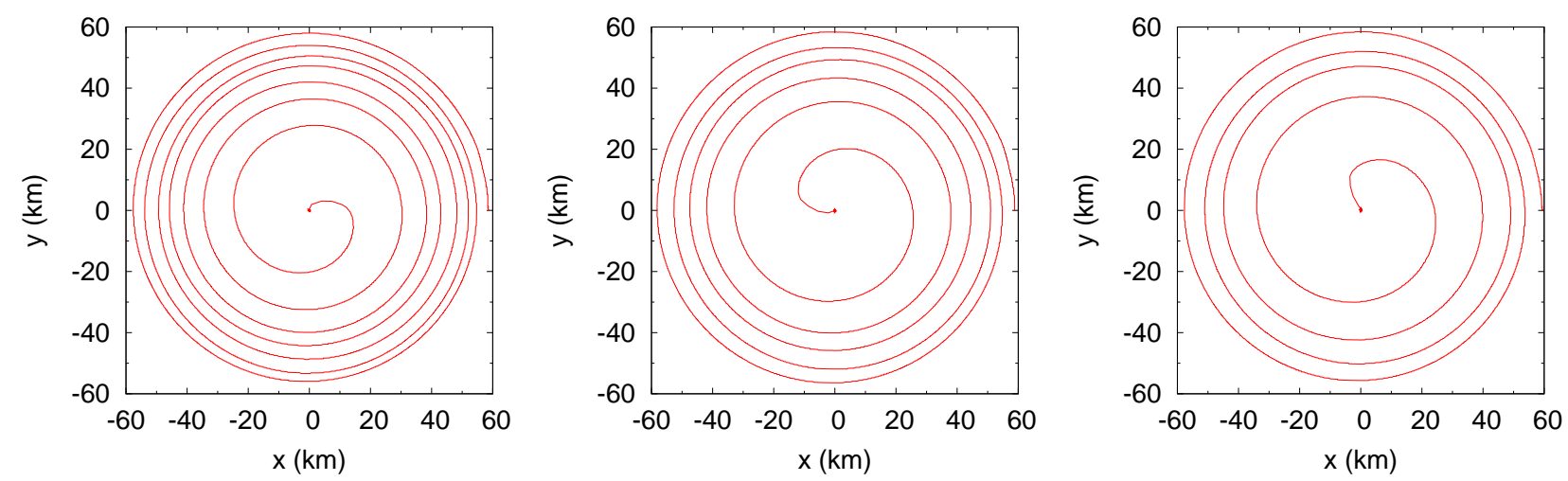

FIG. 1. Evolution of the orbital separation $x_{\mathrm{sep}}^{i}=x_{\mathrm{NS}}^{i}-x_{\mathrm{BH}}^{i}$ of binaries with $\left(Q, M_{\mathrm{NS}}\right)=\left(2,1.35 M_{\odot}\right)$ and HB EOS. The left, middle, and right panels show the results with the prograde $\mathrm{BH}$ spin $a=0.5$, zero $\mathrm{BH}$ spin $a=0$, and retrograde $\mathrm{BH}$ spin $a=-0.5$, respectively.
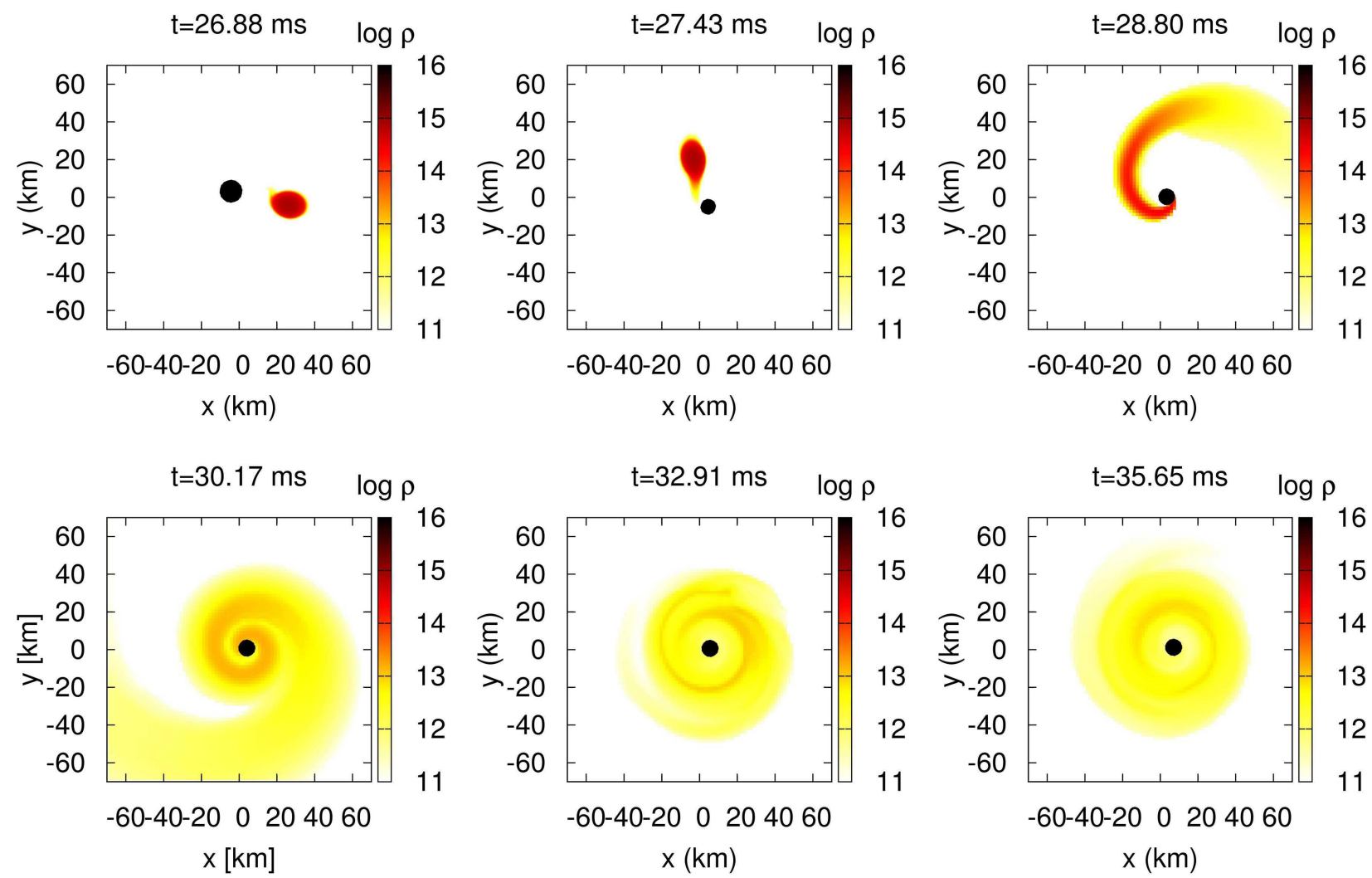

FIG. 2. Evolution of the rest-mass density profile in units of $\mathrm{g} / \mathrm{cm}^{3}$ and the location of the AH on the equatorial plane for model HB-Q3M135a75. The filled circle denotes the region inside the AH. The color (gradational) panel on the right of each plot show $\log _{10}(\rho)$. 

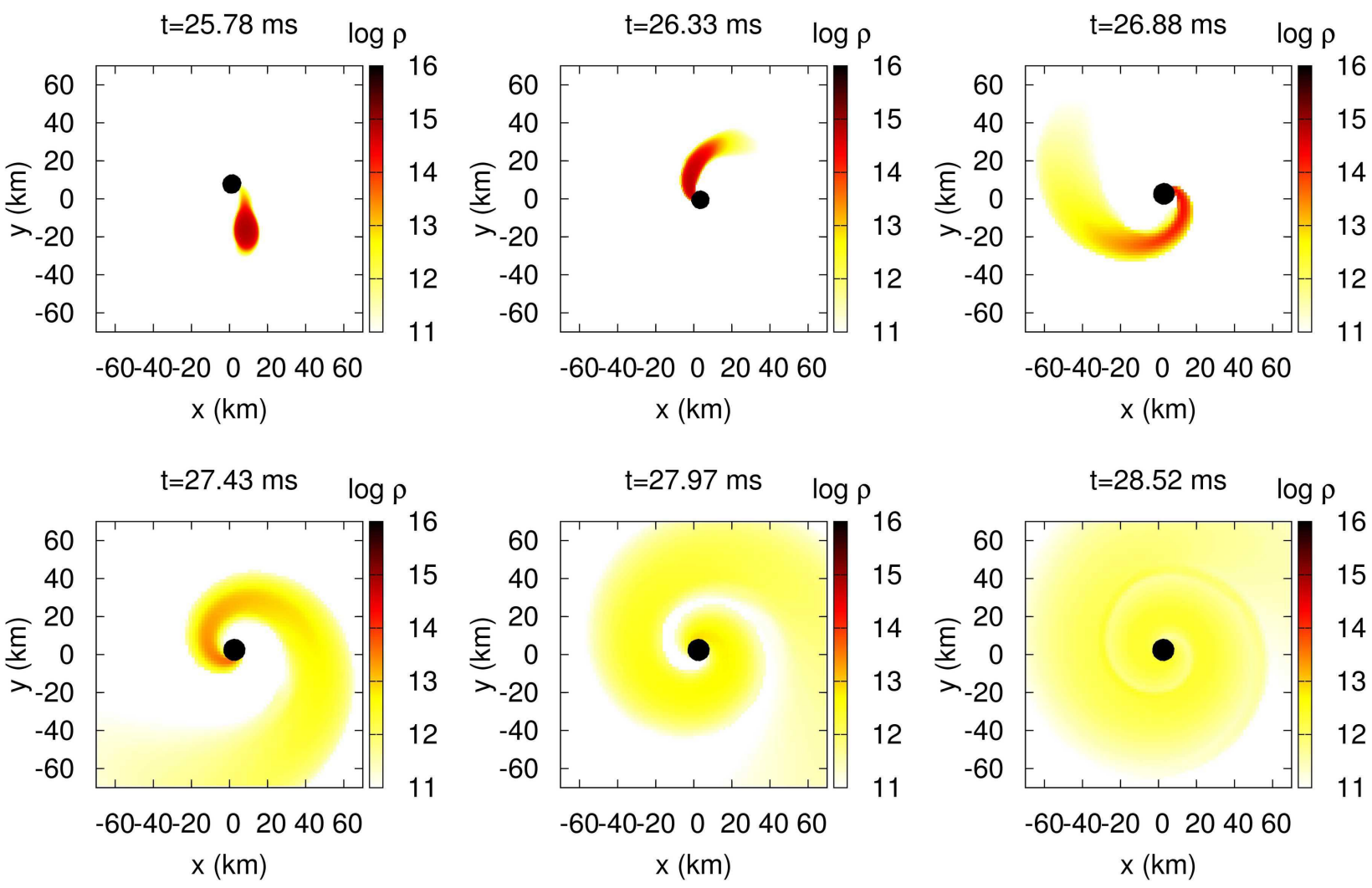

FIG. 3. The same as Fig. 2 but for model HB-Q3M135a5.
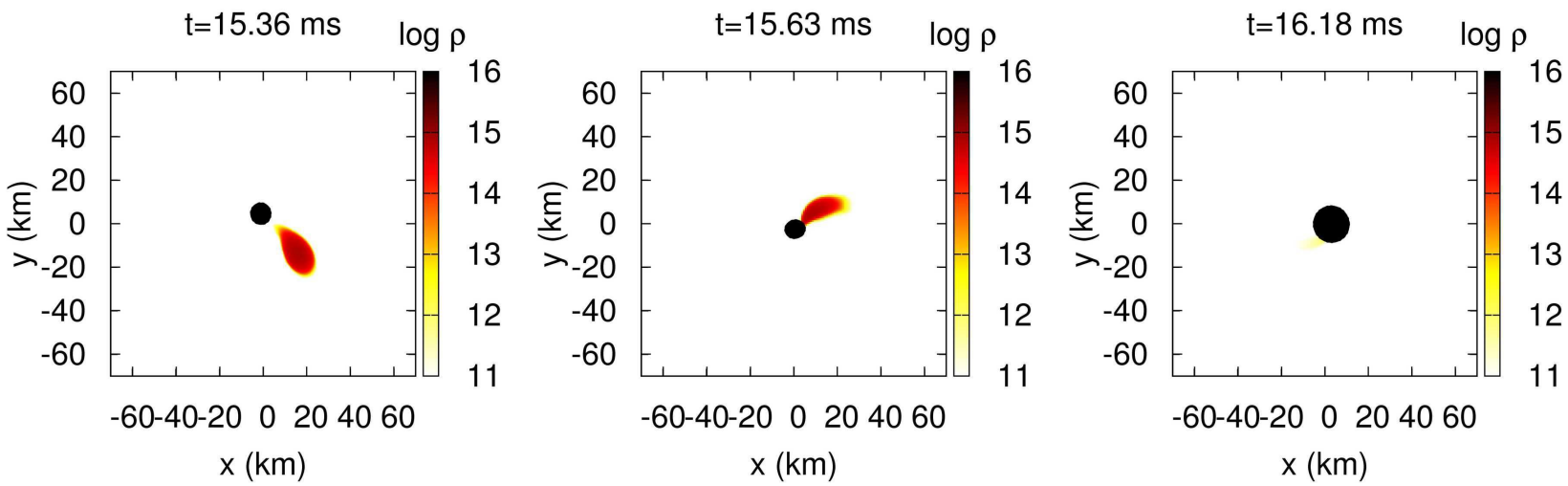

FIG. 4. The same as Fig. 2 but for model HB-Q3M135a-5.

plane at selected time slices for models HB-Q3M135a75, HB-Q3M135a5, and HB-Q3M135a-5 in Figs. 2 4, respectively. The NS is disrupted outside the ISCO in the $a>0$ cases (Figs. 2 and 3) and forms a one-armed spiral structure with a large angular momentum. The material in the inner part of the spiral arm gradually falls onto the $\mathrm{BH}$ due to angular momentum transport via hydrodynamic torque in the spiral arm. The material with a sufficiently large specific angular momentum escapes the capture by the $\mathrm{BH}$ and forms an accretion disk, which survives for a time much longer than the dynamical time scale $\sim$ a few ms. We note that the prompt infall of the one-armed spiral structure onto the $\mathrm{BH}$ occurs from a relatively narrower region for $a=0.5$ than for $a=0.75$. The reason is that the inner edge of the spiral arm contacts the $\mathrm{AH}$ well before the arm becomes nearly axisymmetric due to a large radius of the AH and ISCO for $a=0.5$. The infall of the disrupted material from a narrow region of the $\mathrm{BH}$ frequently occurs when the NS is tidally disrupted in a binary with a high mass ratio, whereas this is rare in a binary with a nonspinning $\mathrm{BH}$ because the NS is not disrupted in a high mass-ratio binary. This 
difference in the merger process is well-reflected in gravitational waveforms (see Sec. IVE). By contrast, the NS is swallowed by the $\mathrm{BH}$ without tidal disruption, and essentially no material is left outside the ISCO for model HB-Q3M135a-5 (Fig. 4).

Note that the feature of the NS tidal disruption appears very weakly not only for model HB-Q3M135a-5 but also for model HB-Q3M135 $(a=0)$ because the mass ratio $Q=3$ is so high that the tidal effect is less important for the nonspinning $\mathrm{BH}$ with the typical NS radius $\sim 11-12 \mathrm{~km}$. The enhancement of the tidal effect by a prograde $\mathrm{BH}$ spin results primarily from the decrease of the $\mathrm{BH}$ ISCO radius [67]. In the Boyer-Lindquist coordinates, a Kerr BH has an ISCO with a smaller radius than a Schwarzschild $\mathrm{BH}$ by a factor of $\geq 1 / 6$, depending on $a$ for a prograde orbit: The ISCO radius approximately halves when the $\mathrm{BH}$ spin increases from $a=0$ to 0.75 . On the other hand, the orbital separation at the onset of mass shedding depends only weakly on the $\mathrm{BH}$ spin in the Boyer-Lindquist coordinates 80 82. This decrease of the ISCO radius enhances the possibility for the disrupted material to escape capture by the $\mathrm{BH}$ and to form a more massive remnant disk than in the nonspinning $\mathrm{BH}$ case. The retrograde $\mathrm{BH}$ spin plays an opposite role; the ISCO radius of the Kerr BH increases by a factor of 1-1.5 for a retrograde orbit, and hence, the tidal effect is less important in the merger process.

Before closing this subsection, we estimate the degree of (undesired) orbital eccentricity in our simulations to assess the circularity of the orbital motion. For this purpose, we compute the evolution of the gauge-invariant orbital angular velocity $\Omega(t)$, which is defined from the $(l, m)=(2,2)$ mode of $\Psi_{4}$ by

$$
\Omega(t)=\frac{1}{2} \frac{\left|\Psi_{4}(l=m=2)\right|}{\left|\int \Psi_{4}(l=m=2) d t\right|} .
$$

The evolution of the orbital angular velocity in our simulation agrees with that derived from the Taylor-T4 formula in the inspiral phase within a small modulation, typically $\Delta \Omega / \Omega \lesssim 5 \%$, which is equivalent to the orbital eccentricity of $\lesssim 3 \%$. This amount of orbital eccentricity is as small as that observed in the nonspinning $\mathrm{BH}$ case with a low mass ratio $Q=2$ [31].

\section{B. Global properties of the disk}

The mass of the remnant disk reflects the significance of NS tidal disruption in a clear way because the disk formation is a result of tidal disruption. A massive disk is formed if tidal disruption of the NS occurs far outside the ISCO. If the mass shedding starts in the vicinity of or inside the ISCO, only a small portion of the mass is left outside the AH. The material is not left outside the $\mathrm{AH}$ when the mass shedding does not occur before the $\mathrm{BH}$ swallows the NS, and the merger of a BH-NS binary may be indistinguishable from that of a $\mathrm{BH}-\mathrm{BH}$ binary except for very small tidal corrections to the inspiral. Thus, the mass of a remnant disk is a reliable indicator of the degree of tidal disruption.

Figure 5 plots the time evolution of the rest mass located outside the $\mathrm{AH}, M_{r>r_{\mathrm{AH}}}$, for $Q=2$ and 3 with different nondimensional $\mathrm{BH}$ spin parameters $a=0.75$, $0.5,0$, and -0.5 . In both plots, $M_{\mathrm{NS}}=1.35 M_{\odot}$ and HB EOS are adopted. We note that the results revised from the previous work [31] are plotted for $a=0$. The dependence of $M_{r>r_{\mathrm{AH}}}$ on $a$ for HB EOS found here is similar to those for other EOSs. We set the time origin to be an approximate merger time $t_{\text {merger }}$. These plots indicate that the mass of the material left outside the $\mathrm{AH}$ relaxes to a quasisteady value for $t-t_{\text {merger }} \gtrsim 3-4 \mathrm{~ms}$, and the relaxed value increases monotonically as the $\mathrm{BH}$ spin increases from retrograde to prograde. This is consistent with the decrease of the BH ISCO radius with the increase of its spin, as described in Sec. IVA. In particular, the remnant disk mass at $\approx 10 \mathrm{~ms}$ after the merger is $\gtrsim 0.1 M_{\odot}$ for all the $\operatorname{EOSs}$ with $(Q, a)=(2, \geq 0.5)$ and $(\leq 4,0.75)$, as shown in Table $\mathrm{V}$ and $\gtrsim 0.05 M_{\odot}$ for $(Q, a)=(3,0.5)$, irrespective of the EOS. The formation of such a massive disk may be encouraging for the $\mathrm{BH}$ NS binary merger hypothesis of a short-hard GRB. For the $a=-0.5$ cases, by contrast, massive accretion disks of $\gtrsim 0.01 M_{\odot}$ are not expected to be formed as merger remnants even for $Q=2$ unless the EOS is extremely stiff (the NS radius is $\approx 15 \mathrm{~km}$ ). This fact indicates that the retrograde $\mathrm{BH}$ spin is unfavorable for producing a central engine of a short-hard GRB.

The prograde $\mathrm{BH}$ spin enhances the disk formation dramatically for a BH-NS binary with a high mass ratio, for which the disk mass is very low when the $\mathrm{BH}$ is nonspinning. We plot the time evolution of $M_{r>r_{\mathrm{AH}}}$ for $Q=4$ and 5 with different EOSs in Fig. 6. In both plots, $M_{\mathrm{NS}}=1.35 M_{\odot}$ and $a=0.75$ are adopted. Figure 6 clearly shows that a massive accretion disk is formed for $Q=4$ and 5 if the $\mathrm{BH}$ has a prograde spin of $a=0.75$. Namely, the formation of a massive accretion disk is universal for the merger of a BH-NS binary with a mass ratio of $Q \lesssim 5$ as far as $a \sim 0.75$ and $M_{\mathrm{NS}}=1.35 M_{\odot}$ (equivalently, $M_{\mathrm{BH}} \lesssim 6.75 M_{\odot}$ ). Note that a heavy $\mathrm{BH}$ of $M_{\mathrm{BH}} \gtrsim 5 M_{\odot}$ is predicted to be realistic as an astrophysical consequence of the stellar evolution with solar metallicity 83] (see, e.g., Ref. [84] for a population synthesis study) and hence as a possible progenitor of the short-hard GRB.

For more quantitative discussion, we plot the disk mass estimated at $\approx 10 \mathrm{~ms}$ after the merger for all the models with $Q=2$ and for models with $(Q, a)=(3, \geq 0)$ as a function of the NS compactness, $\mathcal{C}$, in Fig. 7 Numerical values of $M_{r>r_{\mathrm{AH}}}$ are shown in Table $\mathrm{V}$, as well as other quantities associated with the merger remnants. For any fixed value of $a$, a negative correlation between $M_{r>r_{\mathrm{AH}}}$ and $\mathcal{C}$ is found to hold in Fig. 7. This correlation indicates that the NS with a larger compactness is less subject to tidal deformation and disruption than the NS with a smaller compactness for any fixed value of $a$. This correlation is expected from the nature of a tidal force as 
$\mathrm{HB} Q=2$

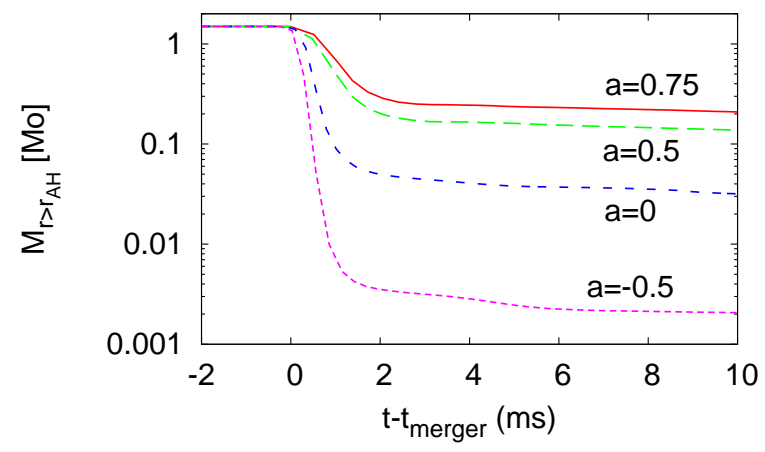

$\mathrm{HB} Q=3$

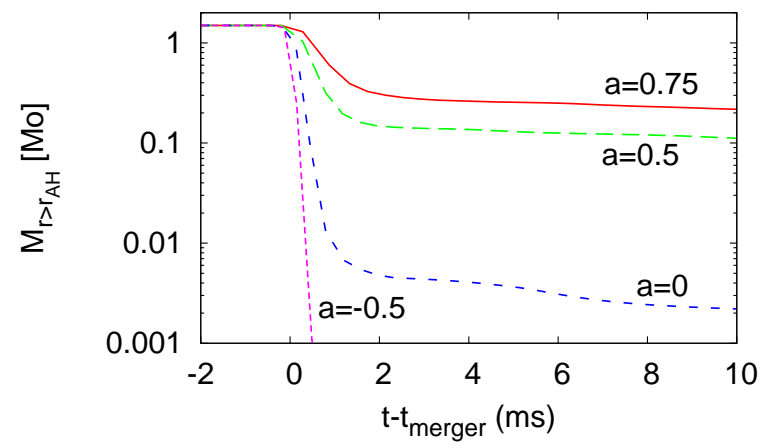

FIG. 5. Evolution of the rest mass of the material located outside an AH of the BH, $M_{r>r_{\mathrm{AH}}}$. The left and right panels show the results for $Q=2$ and 3, respectively. In both plots, $M_{\mathrm{NS}}=1.35 M_{\odot}$ and HB EOS are adopted. The results revised from the previous paper [31] are plotted for $a=0$.
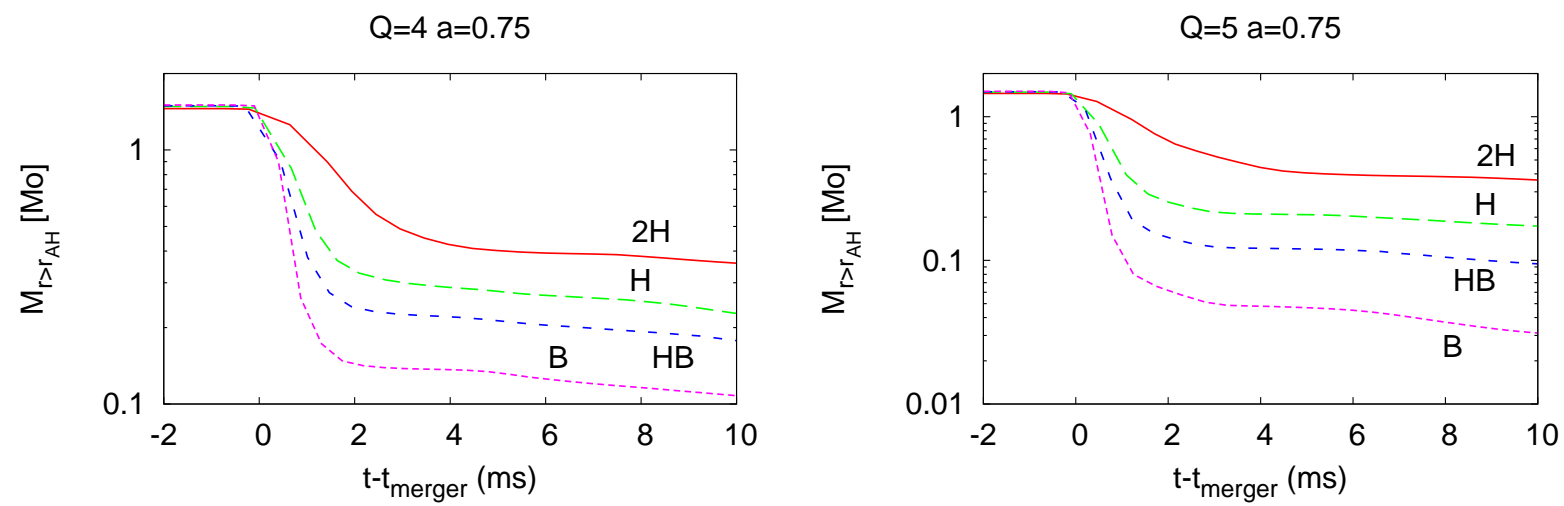

FIG. 6. The same as Fig. 5 but for different models $Q=4$ (left) and 5 (right). In both plots, $M_{\mathrm{NS}}=1.35 M_{\odot}$ and $a=0.75$ are adopted.
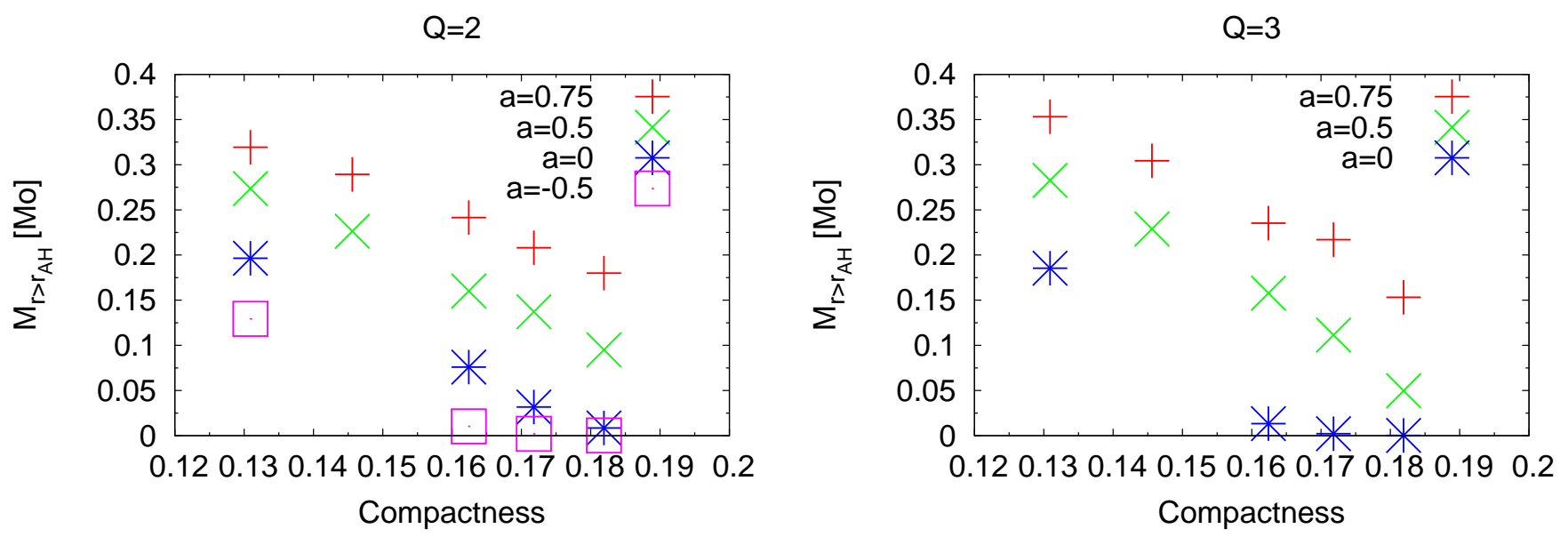

FIG. 7. The disk mass $M_{r>r_{\mathrm{AH}}}$ at $\approx 10 \mathrm{~ms}$ after the onset of the merger as a function of the NS compactness $\mathcal{C}$. The left and right panels show the results for $Q=2$ (left) and $Q=3$ (right). The results revised from the previous work [31] are plotted for $\mathrm{a}=0$. 
a finite-size effect, as found in the study of nonspinning BH-NS binaries [31]. On the other hand, Fig. [7 again shows that the prograde $\mathrm{BH}$ spin increases the disk mass for any fixed value of $\mathcal{C}$. A remarkable fact is that the disk mass does not decrease steeply to a value of $\ll 0.1 M_{\odot}$ as the compactness increases for binaries with $(Q, a)=$ $(\leq 3, \geq 0.5)$. We expect that the coalescence of a BH-NS binary with $(Q, a)=(\leq 3, \geq 0.5)$ may always produce a remnant disk of $\gtrsim 0.01 M_{\odot}$ within a plausible range of the NS compactness, $\mathcal{C} \lesssim 0.2$, although it is possible only if $\mathcal{C} \lesssim 0.18$ for $(Q, a)=(2,0)$ and $\mathcal{C} \lesssim 0.16$ for $(Q, a)=(2,-0.5)$ or $(3,0)$.

The dependence of the disk mass on the NS compactness is different for different values of the mass ratio. We plot in Fig. 8 the disk mass as a function of the NS compactness as in Fig. [7. but for $a=0.75$ and 0.5. This figure shows that the disk mass depends more strongly on $\mathcal{C}$ when the mass ratio, $Q$, is larger. The disk mass is larger for smaller values of $Q$ when the EOS is soft and $\mathcal{C} \gtrsim 0.16$, except for HB-Q2M135a75 and HB-Q3M135a75, for which the disk masses depend only weakly on $Q$. This dependence on $Q$ is expected from the comparison between the mass-shedding radius, $r_{\text {shed, }}$ and the ISCO radius, $r_{\mathrm{ISCO}}$,

$$
\frac{r_{\text {shed }}}{r_{\text {ISCO }}} \propto \mathcal{C}^{-1} Q^{-2 / 3}
$$

where we assume Newtonian gravity for simplicity. This relation states that a larger amount of mass can escape the capture by the $\mathrm{BH}$ and can form an accretion disk when $Q$ is small because the mass shedding sets in at relatively more distant orbit. However, the disk mass may be larger for larger values of $Q$ when the EOS is stiff as $\mathcal{C} \lesssim 0.15$ for $a \geq 0.5$ and $2 \lesssim Q \lesssim 5$. This should be ascribed to the redistribution process of the specific angular momentum of the NS to the disrupted material and to subsequent behavior of the material (such as collision of the fluid elements in spiral arms). This feature suggests that a binary with a larger value of $Q$, say $Q \gtrsim 6$, possibly form a massive remnant disk of $\gtrsim$ $0.1 M_{\odot}$ and could be a progenitor of a short-hard GRB if the EOS is stiff and the BH has a large spin $\gtrsim 0.5$.

To clarify the dependence of the disk mass on the $\mathrm{BH}$ spin, we plot the disk mass as a function of $a$ in Fig. 9 . The EOS (and, equivalently, $\mathcal{C}$ ) is the same for each plot. Again, we find a monotonic and steep increase of the disk mass as the increase of $a$ for the fixed EOS and mass ratio. The enhancement of the disk mass by a prograde spin is more dramatic for the compact NS (for the soft EOS). For example, the difference in the disk mass between the cases of $a=0.75$ and -0.5 is only by a factor of $\sim 3$ when $Q=2$ and $2 \mathrm{H}$ EOS is adopted. This low amplification is natural because tidal disruption of a large NS occurs at an orbit far enough from the ISCO for a substantial amount of the disrupted material to escape the capture by the $\mathrm{BH}$ irrespective of $a$ and because at such a large orbital separation the spin-orbit coupling effect is relatively weak. On the other hand, a few-orders-of- magnitude amplification of the disk mass is seen when $Q=2$ and HB EOS is adopted.

Finally, we comment on a possible unbound outflow. To estimate the rest mass of unbound material, we compute

$$
M_{\mathrm{ub}} \equiv \int_{r>r_{\mathrm{AH}}} \rho_{*} H\left(-u_{t}-1\right) d^{3} x,
$$

where $H(x)$ is a step function. Here, the material with $u_{t}<-1$ should be considered to have an unbound orbit. We find that $M_{\mathrm{ub}}$ can be larger than $0.01 M_{\odot}$ at $\approx 10$ ms after the merger for the stiff EOS like $2 \mathrm{H}$ and $\mathrm{H}$, and $a \geq 0$. However, $M_{\mathrm{ub}}$ does not approach a constant value and rather continues to decrease. Therefore, it is unclear whether $M_{\mathrm{ub}}$ estimated at $10 \mathrm{~ms}$ after the merger can really become unbound or not, and we do not show the precise values of $M_{\mathrm{ub}}$. When the EOS is not stiff, $M_{\mathrm{ub}}$ is negligible within the accuracy of our simulations.

\section{Structure of the remnant disk}

The structure of the remnant disk and its time evolution process depend on the mass ratio of the binary. We plot the rest-mass density profile at $\approx 5$ and $10 \mathrm{~ms}$ after the onset of the merger for binaries with $a=0.75$, HB EOS, and different values of $Q$ in Fig. 10. The left column of Fig. 10 is plotted for $\approx 5 \mathrm{~ms}$ after the onset of the merger and shows that the dense material of $\rho \gtrsim 10^{9} \mathrm{~g} / \mathrm{cm}^{3}$ always extends to $\gtrsim 400 \mathrm{~km}$. The spiral arm always spreads to a far region irrespective of EOSs, as far as the tidal disruption results in a massive disk. These plots also suggest that the accretion disk for a large value of $Q$ - say, $Q=5$ - keeps a nonaxisymmetric structure in the vicinity of the remnant $\mathrm{BH}$ at this time. This feature is qualitatively the same for binaries with other EOSs. When $Q$ is small as $\sim 2$, the accretion disk becomes nearly axisymmetric in $\approx 5 \mathrm{~ms}$ after tidal disruption because the dynamical time scale of the system (which is proportional to the $\mathrm{BH}$ mass) is shorter for a smaller value of $Q$. Also, because the ISCO radius of the $\mathrm{BH}$ is smaller, the maximum rest-mass density, $\rho_{\max }$, of the disk (which should be approximately proportional to the inverse square of the $\mathrm{BH}$ mass) reaches a higher value on average in time for a smaller value of $Q$. It should be noted that the difference in $\rho_{\max }$ comes primarily from the difference in the radius and not from the difference in the disk mass, which do not vary by an order of magnitude for $a=0.75$ and $Q=2-5$. This difference in the nonaxisymmetric structure results in different features of gravitational waves (see Sec. IVE).

The middle and right columns of Fig. 10 plot snapshots at $\approx 10 \mathrm{~ms}$ after the onset of the merger. At this time, nonaxisymmetric structures are not as significant as those at $\approx 5 \mathrm{~ms}$ after the onset of the merger because the accretion disk settles toward an approximately stationary state in the vicinity of the BH. The maximum values of the rest-mass density, $\rho_{\max }$, in the accretion 
TABLE V. Several key quantities for the merger remnants for $M_{\mathrm{NS}}=1.35 M_{\odot}$. All the quantities are estimated at $\approx 10 \mathrm{~ms}$ after the approximate merger time $t=t_{\text {merger }} . M_{r>r_{\mathrm{AH}}}$ is the rest mass of the disk surrounding the $\mathrm{BH}$; because the accretion is still ongoing due to the hydrodynamic angular momentum transport process, the listed values only give approximate masses of the long-lived accretion disks, which survive for a time longer than the dynamical time scale $\sim$ a few ms. $C_{e}$ and $C_{p}$ are the circumferential radii of the $\mathrm{AH}$ along the equatorial and meridional planes, respectively. $C_{e} / 4 \pi$ and $M_{\mathrm{BH}, \mathrm{c}}$ denote approximate masses of the remnant BH. $M_{\mathrm{irr}, \mathrm{f}}$ is the irreducible mass of the remnant $\mathrm{BH}$, and $a_{\mathrm{f}}$ is the nondimensional spin parameter of the remnant $\mathrm{BH}$ estimated from $C_{p} / C_{e} . a_{\mathrm{f} 2}$ and $a_{\mathrm{f} 1}$ are also the nondimensional spin parameters, estimated from the quantities on the $\mathrm{AH}$ and approximate conservation laws, respectively. We note that the values associated with the remnant $\mathrm{BH}$ for model B-Q2M135a75 (with an asterisk) are evaluated at $\approx 5 \mathrm{~ms}$ after the onset of the merger because the BH area decreases by $\gtrsim 1 \%$ at $\approx 10 \mathrm{~ms}$ after the onset of the merger and the error becomes large.

\begin{tabular}{|c|c|c|c|c|c|c|c|c|}
\hline Model & $M_{r>r_{\mathrm{AH}}}\left[M_{\odot}\right]$ & $C_{e} / 4 \pi M_{0}$ & $M_{\mathrm{BH}, \mathrm{c}} / M_{0}$ & $M_{\mathrm{irr}, \mathrm{f}} / M_{0}$ & $C_{p} / C_{e}$ & $a_{\mathrm{f}}$ & $a_{\mathrm{f} 2}$ & $a_{\mathrm{f} 1}$ \\
\hline 2H-Q2M135a75 & 0.32 & 0.913 & 0.915 & 0.789 & 0.807 & 0.87 & 0.87 & 0.95 \\
\hline 1.5H-Q2M135a75 & 0.29 & 0.918 & 0.920 & 0.785 & 0.794 & 0.89 & 0.89 & 0.95 \\
\hline H-Q2M135a75 & 0.24 & 0.927 & 0.929 & 0.783 & 0.780 & 0.91 & 0.90 & 0.94 \\
\hline HB-Q2M135a75 & 0.21 & 0.933 & 0.934 & 0.783 & 0.772 & 0.91 & 0.91 & 0.94 \\
\hline B-Q2M135a75 & 0.18 & $0.937^{*}$ & 0.938 & $0.790^{*}$ & $0.778^{*}$ & $0.91^{*}$ & $0.91^{*}$ & 0.93 \\
\hline 2H-Q2M135a5 & 0.27 & 0.925 & 0.926 & 0.825 & 0.843 & 0.81 & 0.81 & 0.84 \\
\hline 1.5H-Q2M135a5 & 0.23 & 0.935 & 0.936 & 0.831 & 0.840 & 0.82 & 0.81 & 0.84 \\
\hline H-Q2M135a5 & 0.17 & 0.945 & 0.946 & 0.837 & 0.836 & 0.82 & 0.82 & 0.84 \\
\hline HB-Q2M135a5 & 0.14 & 0.951 & 0.952 & 0.840 & 0.832 & 0.83 & 0.83 & 0.84 \\
\hline B-Q2M135a5 & 0.095 & 0.959 & 0.960 & 0.846 & 0.830 & 0.83 & 0.83 & 0.84 \\
\hline 2H-Q2M135a-5 & 0.13 & 0.9 & 0.962 & 0.931 & 0.954 & 0.48 & 0.48 & 0.50 \\
\hline H-Q2M13 & 0.010 & 0.985 & 0.986 & 0.950 & 0.948 & 0.51 & 0.51 & 0.52 \\
\hline HB-Q2M135a-5 & 0.0021 & 0.985 & 0.986 & 0.952 & 0.950 & 0.50 & 0.50 & 0.51 \\
\hline B-Q2M135a-5 & $2 \times 10^{-4}$ & 0.983 & 0.984 & 0.952 & 0.952 & 0.49 & 0.49 & 0.50 \\
\hline 2H-Q3M13 & 0.35 & 0.927 & 0.927 & 0.807 & 0.815 & 0.86 & 0.86 & 0.90 \\
\hline 1.5H-Q3M135a75 & 0.30 & 0.9 & 0.934 & 0.811 & 0.815 & 0.86 & 0.86 & 0.90 \\
\hline H-Q3M135a75 & 0.24 & 0.939 & 0.943 & 0.820 & 0.818 & 0.85 & 0.85 & 0.91 \\
\hline HB-Q3M135a75 & 0.22 & 0.941 & 0.943 & 0.812 & 0.805 & 0.87 & 0.87 & 0.90 \\
\hline B-Q3M135a75 & 0.15 & 0.949 & 0.951 & 0.824 & 0.812 & 0.86 & 0.86 & 0.89 \\
\hline 2H-Q3M135a5 & 0.28 & 0.939 & 0.940 & 0.858 & 0.874 & 0.74 & 0.74 & 0.77 \\
\hline 1.5H-Q3M135a5 & 0.23 & 0.946 & 0.948 & 0.862 & 0.871 & 0.75 & 0.75 & 0.78 \\
\hline H-Q3M135a5 & 0.16 & 0.955 & 0.957 & 0.867 & 0.866 & 0.76 & 0.76 & 0.78 \\
\hline HB-Q3M135a5 & 0.11 & 0.961 & 0.963 & 0.871 & 0.864 & 0.77 & 0.77 & 0.78 \\
\hline B-Q3M135a5 & 0.050 & 0.969 & 0.971 & 0.877 & 0.862 & 0.77 & 0.77 & 0.79 \\
\hline HB-Q3M135a-5 & $<10^{-4}$ & 0.986 & 0.987 & 0.973 & 0.980 & 0.32 & 0.32 & 0.33 \\
\hline 2H-Q4M135a75 & 0.36 & 0.937 & 0.938 & 0.825 & 0.828 & 0.84 & 0.84 & 0.87 \\
\hline H-Q4M135a75 & 0.23 & 0.948 & 0.951 & 0.831 & 0.823 & 0.84 & 0.84 & 0.88 \\
\hline HB-Q4M135a75 & 0.18 & 0.953 & 0.956 & 0.833 & 0.821 & 0.85 & 0.85 & 0.88 \\
\hline B-Q4M135a75 & 0.11 & 0.960 & 0.963 & 0.837 & 0.817 & 0.85 & 0.85 & 0.88 \\
\hline 2H-Q4M135a5 & 0.28 & 0.950 & 0.951 & 0.879 & 0.891 & 0.70 & 0.70 & 0.72 \\
\hline H-Q4M135a5 & 0.085 & 0.970 & 0.973 & 0.890 & 0.880 & 0.73 & 0.73 & 0.74 \\
\hline HB-Q4M135a5 & 0.024 & 0.976 & 0.979 & 0.894 & 0.878 & 0.74 & 0.74 & 0.75 \\
\hline B-Q4M135a5 & 0.0034 & 0.978 & 0.980 & 0.896 & 0.878 & 0.74 & 0.74 & 0.75 \\
\hline 2H-Q5M135a75 & 0.36 & 0.946 & 0.947 & 0.838 & 0.835 & 0.82 & 0.82 & 0.85 \\
\hline H-Q5M135a75 & 0.17 & 0.960 & 0.963 & 0.844 & 0.827 & 0.84 & 0.84 & 0.86 \\
\hline HB-Q5M135a75 & 0.095 & 0.966 & 0.970 & 0.848 & 0.824 & 0.84 & 0.84 & 0.86 \\
\hline B-Q5M135a75 & 0.031 & 0.972 & 0.975 & 0.851 & 0.821 & 0.85 & 0.85 & 0.87 \\
\hline
\end{tabular}



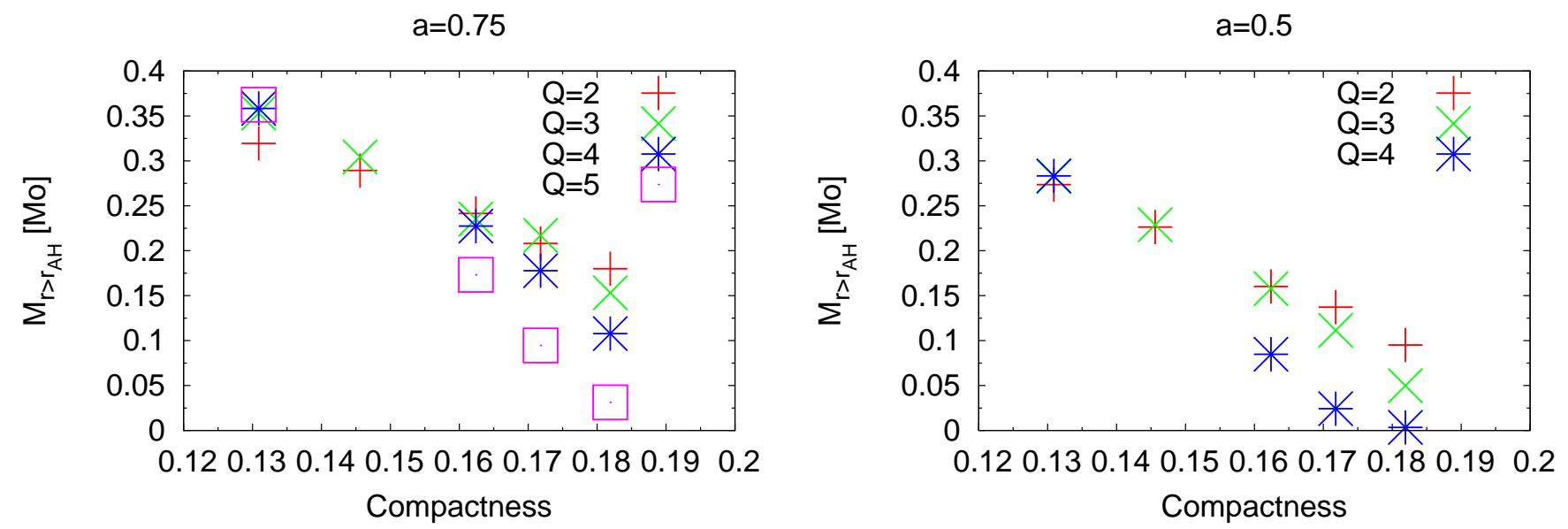

FIG. 8. The same as Fig. 7 but for $a=0.75$ and $a=0.5$ with $Q=2-5$.
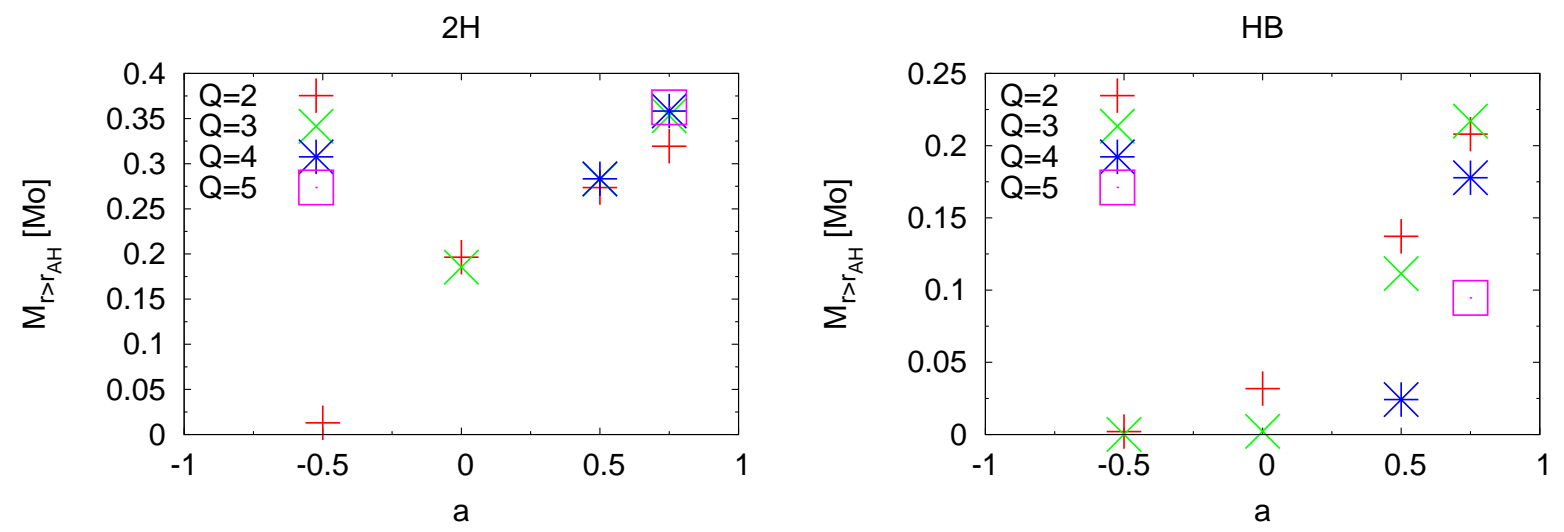

FIG. 9. The disk mass, $M_{r>r_{\mathrm{AH}}}$, at $\approx 10 \mathrm{~ms}$ after the onset of the merger as a function of the nondimensional spin parameter of the $\mathrm{BH} a$ for models with $M_{\mathrm{NS}}=1.35 M_{\odot}$. The left and right panels show the results for models with the $2 \mathrm{H}$ and HB EOSs, respectively. The results revised from the previous work [31] are plotted for $a=0$. 
disk are still higher for a smaller value of $Q$. Indeed, the right column of Fig. 10] shows that smaller values of $Q$ result in producing a wider region with $\rho>10^{12} \mathrm{~g} / \mathrm{cm}^{3}$. By contrast, the disk for $Q=5$ does not have such a highdensity region. The smaller density may be unfavorable to be the short-hard GRB model.

The size of a region where $\rho>10^{10} \mathrm{~g} / \mathrm{cm}^{3}$ coincides approximately among four models with different values of $Q$ and is always $\sim 100 \mathrm{~km}$. Furthermore, the middle column suggests that the region of $\rho>10^{8} \mathrm{~g} / \mathrm{cm}^{3}$ extends to larger distances when $Q$ is larger. We plot the radial distribution of $\rho$ along $x$ and $y$ axes for these models in Fig. 11. Note that low-density regions near the origin are inside the $\mathrm{BH}$. These plots show that $\rho_{\max }$ is systematically higher for the binary with a smaller value of $Q$. These also show that the location of the isodensity surface of $\rho=10^{10} \mathrm{~g} / \mathrm{cm}^{3}$ approximately coincides among different values of $Q$. Taking these facts into account, we conclude that a typical profile of $\rho(r)$ is steeper for smaller values of $Q$ in the vicinity of the $\mathrm{BH}$. A region of $\gtrsim 100 \mathrm{~km}$ away from the $\mathrm{BH}$, where the profile $\rho(r)$ shows relatively shallow decrease and $\lesssim 10^{10} \mathrm{~g} / \mathrm{cm}^{3}$, corresponds to the tail component, as is seen in the middle column of Fig. 10.

\section{Properties of the remnant $\mathrm{BH}$}

Table $\nabla$ shows that masses and nondimensional spin parameters of the remnant BHs depend weakly on the adopted EOSs. The mass of the remnant $\mathrm{BH}$ tends to become large as the EOS softens for fixed values of $\left(Q, M_{\mathrm{NS}}, a\right)$ for the case in which tidal disruption of the NS occurs. The reason for this is that the tidal disruption occurs near the ISCO, and then the BH swallows a large amount of the NS mass when the EOS is soft. Exceptionally, the mass of the $\mathrm{BH}$ becomes slightly larger for $\mathrm{H}$ and $\mathrm{HB}$ EOSs than for B EOS for binaries with $(Q, a)=(2,-0.5)$. The reason for this is that the remnant disk masses are small as $\lesssim 0.01 M_{\odot}$ for these cases and the amount of the energy radiated by gravitational waves primarily determines the final state (for more compact NSs, the radiated energy is larger because a closer inspiral orbit is achieved). The spin angular momentum of the remnant $\mathrm{BH} S_{\mathrm{BH}, \mathrm{f}}$ shows similar behavior to that of the $\mathrm{BH}$ mass. The situation becomes complicated for a spin parameter of the remnant $\mathrm{BH}$ defined by $a_{\mathrm{f}}=S_{\mathrm{BH}, \mathrm{f}} / M_{\mathrm{BH}, \mathrm{f}}^{2}$; the competition between the mass and angular momentum losses from the system makes the dependence of the nondimensional spin parameter of the remnant $\mathrm{BH}$ on the EOS very weak. For comparison, $a_{\mathrm{f} 1}$ and $a_{\mathrm{f} 2}$ defined in Sec. IIIB are also shown in Table V. As is found in our previous work [31], $a_{\mathrm{f}}$ and $a_{\mathrm{f} 2}$ agree with each other within the error of $\Delta a=0.003$. By contrast, $a_{\mathrm{f} 1}$ does not agree well with the other two estimates, as is found in another previous work of ours [29], particularly when the massive remnant disk is formed and/or the mass of the $\mathrm{BH}$ is small: the maximum er- ror is $\Delta a \approx 0.08$. Taking into account the fact that the agreement between $C_{e} / 4 \pi$ and $M_{\mathrm{BH}, \mathrm{f}}$ is always better than $0.5 \%$, a possible reason for this discrepancy is that Eq. (31) systematically underestimates the angular momentum of the disk. Hereafter, we only refer to $a_{\mathrm{f}}$ as the nondimensional spin parameter of the remnant $\mathrm{BH}$.

The nondimensional spin parameter of the remnant $\mathrm{BH}$ depends strongly on the initial spin parameter, $a$, and the mass ratio, $Q$. Approximate values of the nondimensional spin parameter of the remnant $\mathrm{BH}, a_{\mathrm{f}}$, are shown in Fig. 12 as a function of the initial $\mathrm{BH}$ spin parameter, $a$. We also plot lines obtained by a linear fitting of data for $Q=2$ and 3 of the following form,

$$
\begin{aligned}
& a_{\mathrm{f}}=0.32 a+0.66(Q=2), \\
& a_{\mathrm{f}}=0.43 a+0.54(Q=3) .
\end{aligned}
$$

The relation for $Q=3$ agrees approximately with the results reported in Refs. [28, 85] within an error of $\lesssim 5 \%$, and the agreement becomes better for a larger value of $a$. Figure 12 and these relations show that $a_{\mathrm{f}}$ is an approximately linear function of $a$. In a zeroth approximation, the slope and intercept of the linear relation denote the contribution from the initial $\mathrm{BH}$ spin angular momentum, $S_{\mathrm{BH}}$, and the orbital angular momentum of the binary, $J_{0}$, respectively. The larger slope for a larger value of $Q$ is explained by a larger contribution from the spin of the initial $\mathrm{BH}$ of mass $M_{\mathrm{BH}}=Q M_{\mathrm{NS}}$ to the spin of the remnant $\mathrm{BH}$ of mass $M_{\mathrm{BH}, \mathrm{f}} \sim(1+Q) M_{\mathrm{NS}}$. These predict the value of the slope to be $Q^{2} /(1+Q)^{2}$. However, the slope obtained by numerical simulations is smaller by $\sim 25-30 \%$ than this predicted slope, because the amount of angular momenta redistributed to the remnant disk and extracted by gravitational waves become larger for a larger value of $a$. The fitting function also suggests that the merger of an extremely spinning $\mathrm{BH}$ of $a=1$ and a NS with an irrotational velocity field results in a remnant $\mathrm{BH}$ with $a_{\mathrm{f}} \approx 0.98$ for BH-NS binaries with $Q=2$ and 3 and hence never forms an overspinning $\mathrm{BH}$, i.e., a $\mathrm{BH}$ with $a_{\mathrm{f}}>1$. Furthermore, the results for $Q=4$ shown in Table $\mathrm{V}$ also suggest $a_{\mathrm{f}} \approx 0.97$ for the merger of an extremely spinning BH and an irrotational NS. These results give a circumstantial support for cosmic censorship conjecture [86]. Whether $a_{\mathrm{f}} \lesssim 0.98(<1)$ is an universal consequence of a general BH-NS binary merger or not should be confirmed by simulations of higher mass-ratio binary mergers, in particular, with (nearly) extremal $\mathrm{BH}$ spin.

From these typical values of $a_{\mathrm{f}}$ and $M_{\mathrm{BH}, \mathrm{f}}$, we can estimate typical frequencies of quasinormal modes (hereafter QNM) $f_{\mathrm{QNM}}$ of the remnant $\mathrm{BH}$ by a fitting formula [87]

$$
f_{\mathrm{QNM}} M_{\mathrm{BH}, \mathrm{f}} \approx \frac{1}{2 \pi}\left[1.5251-1.1568(1-a)^{0.1292}\right]
$$

We plot these values in Fig. 13. They are in good agreement with those of the ringdown waveforms, if the QNM is excited after the merger. 

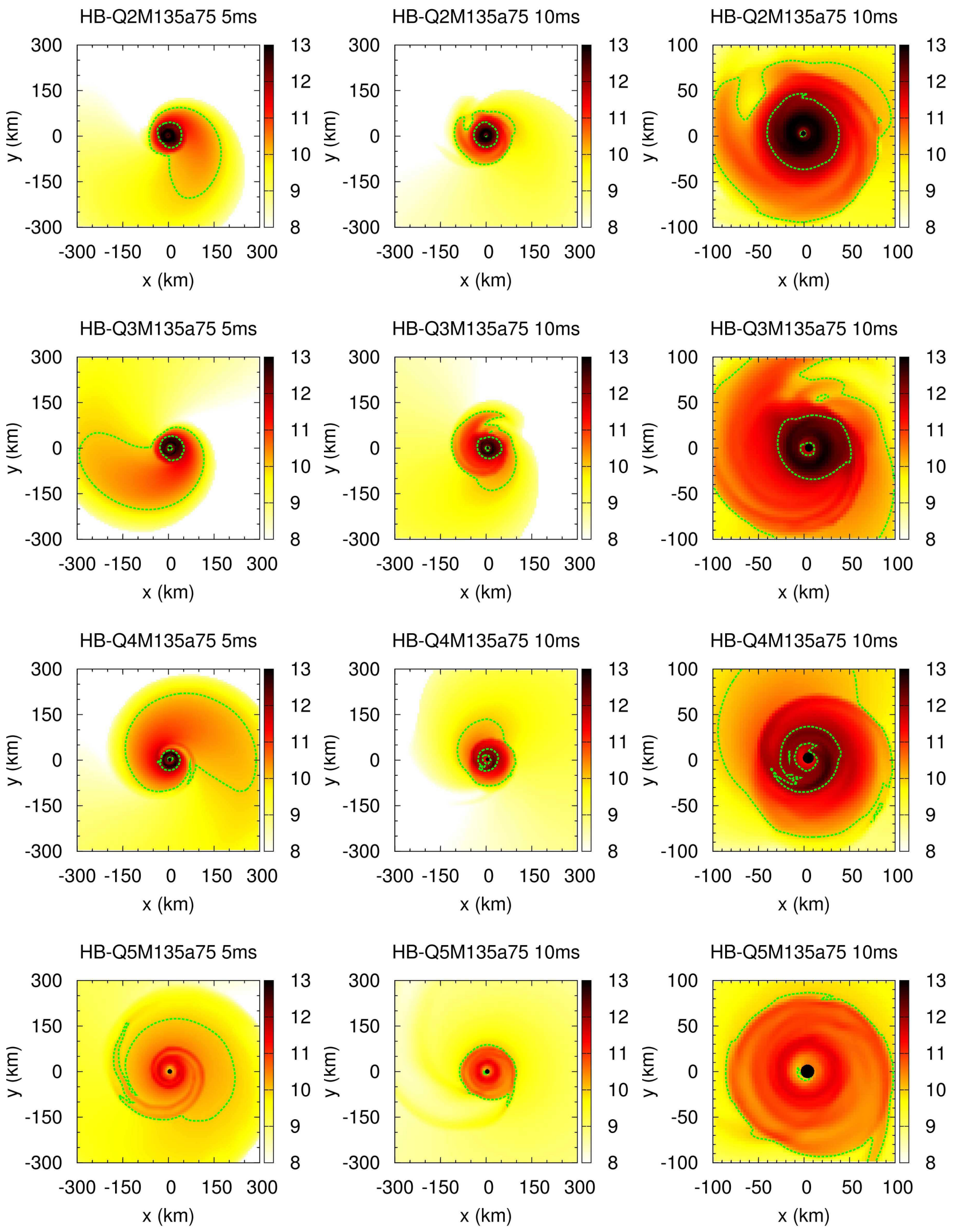

FIG. 10. The same as Fig. 2 with contour curves for $\rho=10^{10}$ and $10^{12} \mathrm{~g} / \mathrm{cm}^{3}$ plotted. In all the plots, HB EOS and $a=0.75$ are adopted. The first, second, third, and fourth rows are for $Q=2,3,4$, and 5 , respectively. The left column plots the snapshots at $5 \mathrm{~ms}$ after the onset of the merger. The middle column plots the snapshots at $10 \mathrm{~ms}$ after the onset of the merger, and the right column plots close-ups of the middle column. 

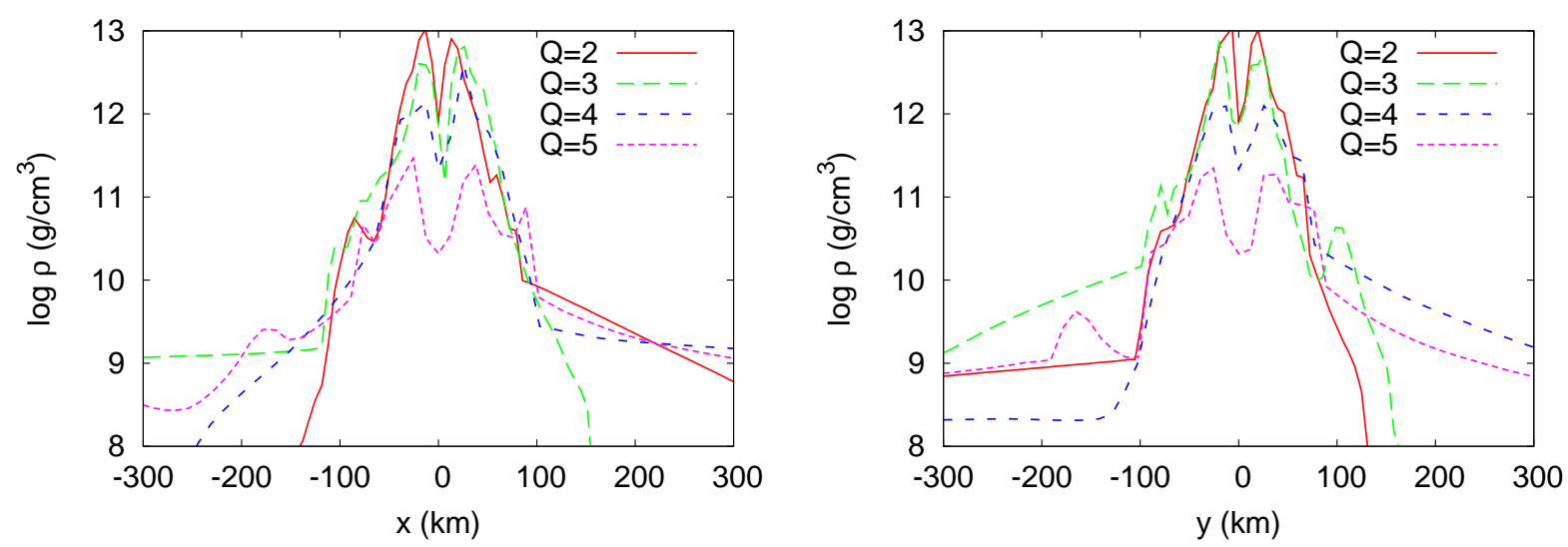

FIG. 11. Radial distribution of the rest-mass density at $10 \mathrm{~ms}$ after the onset of the merger for different values of $Q$. The left and right panels show the distribution along the $x$ and $y$ axes, respectively. In both plots, $\left(M_{\mathrm{NS}}, a\right)=\left(1.35 M_{\odot}, 0.75\right)$ and $\mathrm{HB}$ EOS are adopted.

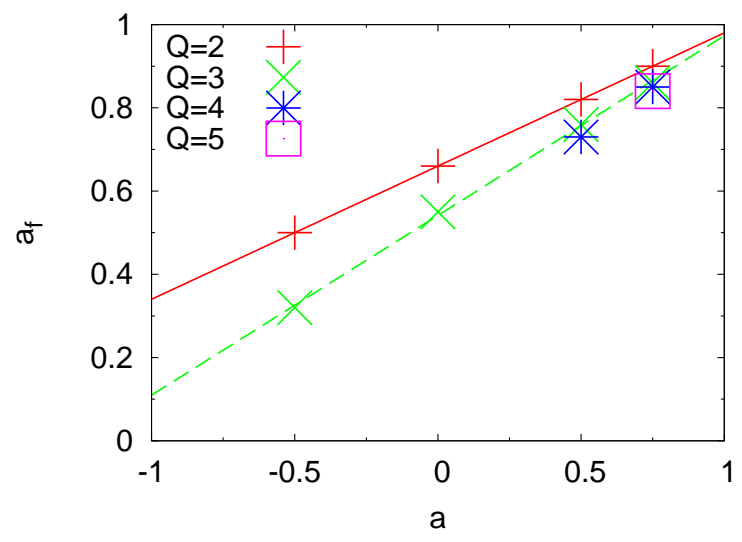

FIG. 12. The typical nondimensional spin parameters of the remnant $\mathrm{BH} a_{\mathrm{f}}$ as a function of the initial $\mathrm{BH}$ spin parameter $a$. The solid lines are obtained by a linear fitting of the data for $Q=2$ and $Q=3$.

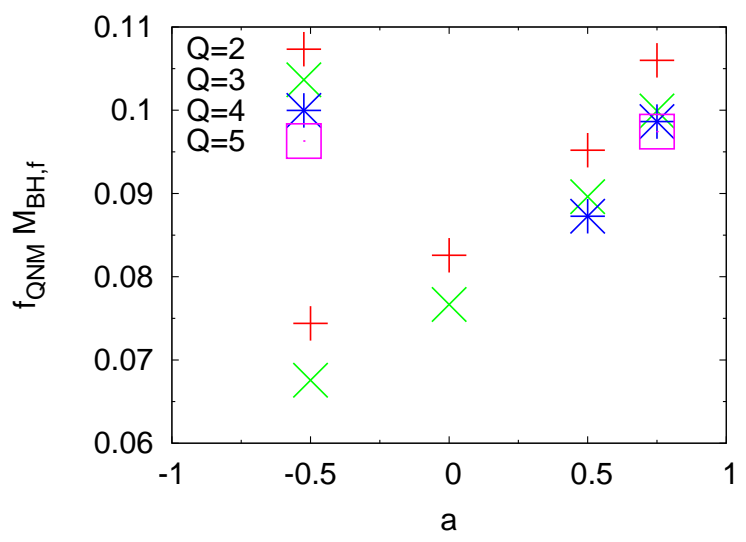

FIG. 13. The typical QNM frequency of the remnant BH normalized by its mass $f_{\mathrm{QNM}} M_{\mathrm{BH}, \mathrm{f}}$.

\section{E. Gravitational waveforms}

In this section, we show $(l, m)=(2,2)$, plus-mode gravitational waveforms $h_{+}$for selected models obtained in this study, as well as the waveform for models obtained in our previous simulations [31]. We plot all the waveforms for an observer along the $z$ axis as a function of the approximate retarded time

$$
t_{\text {ret }}=t-D-2 M_{0} \ln \left(D / M_{0}\right) .
$$

The amplitude of the waveforms is normalized as $D h_{+} / m_{0}$ or we show physical amplitude observed at a hypothetical distance $D=100 \mathrm{Mpc}$ along the $z$ axis. Gravitational waveforms calculated in the Taylor-T4 formula are plotted together in the figures to validate the waveforms obtained in our numerical simulations during the inspiral phase. Numerical waveforms during $2-3$ initial cycles deviate from ones obtained from the Taylor-T4 formula in all the cases due to the lack of an approaching velocity in the initial data. This deficit is ascribed to insufficient modeling of the quasiequilibrium state and improvement in the future is important to obtain more accurate gravitational-wave templates [88, 89]. Our waveforms also deviate from the Taylor-T4 waveforms in the late inspiral phase due to a physical reason, which we describe below. Comparisons between waveforms obtained from simulations with different grid resolutions are shown in the Appendix

Figure 14 shows the gravitational waveforms for binaries with $\mathrm{HB}$ EOS, $\left(Q, M_{\mathrm{NS}}\right)=\left(3,1.35 M_{\odot}\right)$ but with different $\mathrm{BH}$ spin parameters, $a=0.75,0.5,0$, and -0.5 . This figure shows that the time to the merger, to which we refer approximately as the time at which the maximum gravitational-wave amplitude is achieved, for $\Omega_{0} m_{0}=0.030$ becomes longer by $\approx 10 \mathrm{~ms}$ as the increase of the $\mathrm{BH}$ spin within the range concerned here. This difference in the merger time owes primarily to the spin-orbit interaction described in Sec. IVA and this 

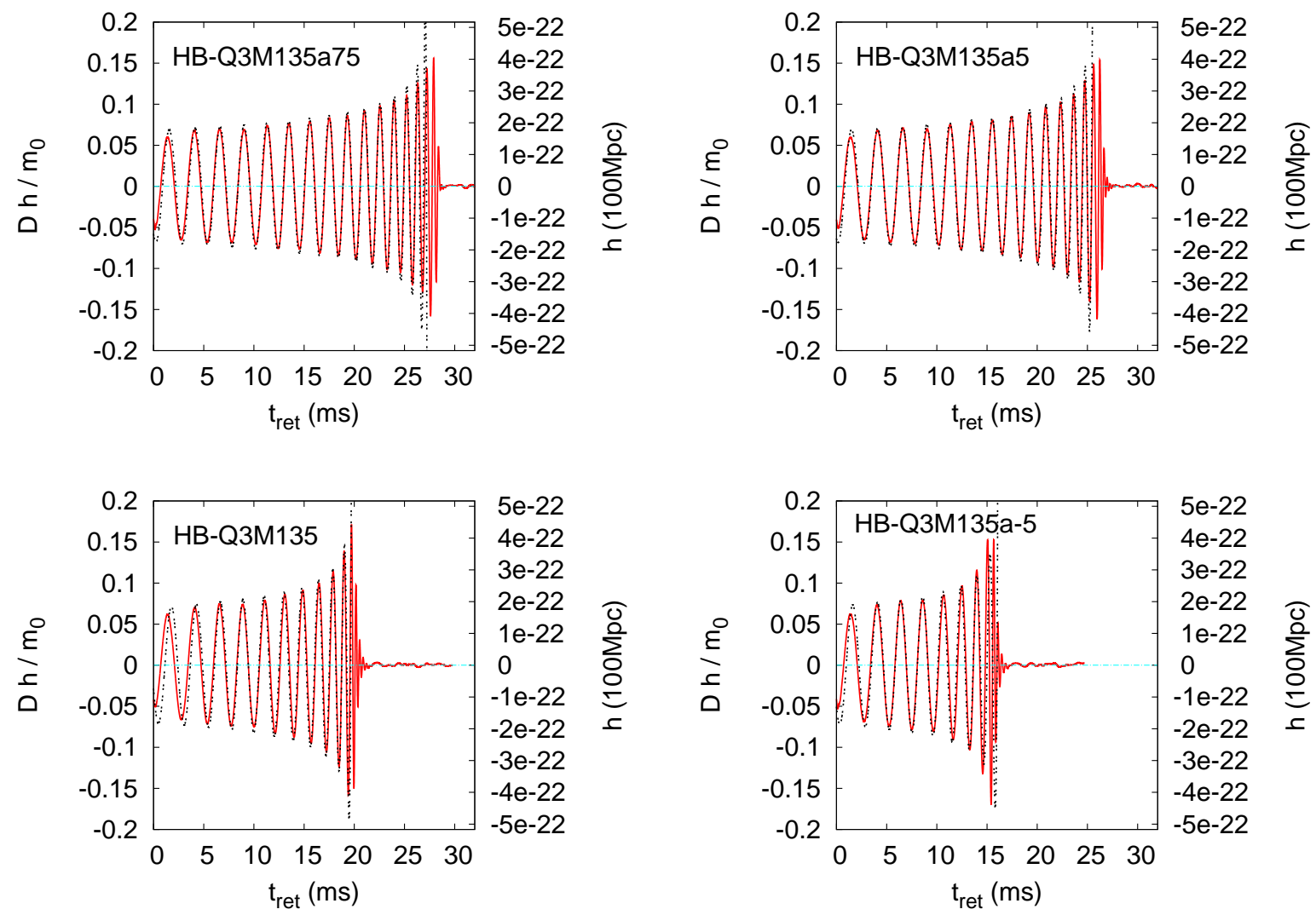

FIG. 14. $(l, m)=(2,2)$, plus-mode gravitational waves for models HB-Q3M135a75, HB-Q3M135a5, HB-Q3M135, and HBQ3M135a-5. All the waveforms are shown for an observer located along the $z$ axis (the axis of rotation) and plotted as a function of a retarded time. The left axis denotes the amplitude normalized by the distance from the binary $D$ and the total mass $m_{0}$. The right axis denotes the physical amplitude of gravitational waves observed at a hypothetical distance 100 Mpc. The dotted curves denote the waveform calculated by the Taylor-T4 formula.

behavior is qualitatively the same for binaries with any EOS. The numerical and Taylor-T4 waveforms agree well with each other during an inspiral phase for all the cases.

For the prograde BH spin cases, the Taylor-T4 formula does not track the evolution for $\sim 0.5$ inspiral orbit just before the merger. The Taylor-T4 amplitude departs from that of numerical relativity and even diverges. Accordingly, the number of gravitational-wave cycles differs by as much as unity between the numerical and Taylor-T4 waveforms. The difference in the number of cycles is larger for higher mass-ratio binaries with prograde $\mathrm{BH}$ spins. We show the waveforms for binaries with $\left(Q, M_{\mathrm{NS}}, a\right)=\left(4,1.35 M_{\odot}, 0.75\right)$ and with $\left(Q, M_{\mathrm{NS}}, a\right)=\left(5,1.35 M_{\odot}, 0.75\right)$ for $2 \mathrm{H}, \mathrm{H}, \mathrm{HB}$, and $\mathrm{B}$ EOSs in Figs. 15] and 16, respectively. The deviation is clear for $\mathrm{H}, \mathrm{HB}$, and $\mathrm{B}$ EOSs in both figures. This difference indicates that the phase evolution predicted by the Taylor-T4 formula is not sufficient to model the last inspiral phase of a coalescing binary with the high mass ratio of $Q \gtrsim 3$ and the prograde $\mathrm{BH}$ spin of $a \gtrsim 0.5$.

For the retrograde $\mathrm{BH}$ spin case (the bottom right panel of Fig. 14), the phase evolution deviates between the numerical and Taylor-T4 waveforms in the last orbit before the merger. This deviation may be partly ascribed to the small number of orbits in our simulation but appears to be primarily ascribed to a larger angular velocity, or equivalently a larger PN parameter, $\Omega m_{0}$, at the last orbit for a retrograde BH spin. Thus, the Taylor-T4 formula seems to be again insufficient for modeling the retrograde $\mathrm{BH}$ spin cases.

Figure 14 also shows that the gravitational waveform in the merger stage depends strongly on the $\mathrm{BH}$ spin. For a binary with $(Q, a)=(3,0.75)$, gravitational waves show a sudden decrease in the amplitude at $t_{\text {ret }} \approx 27 \mathrm{~ms}$, which is a clear signature of tidal disruption. Gravitational waves associated with the ringdown of a remnant $\mathrm{BH}$ are absent due to the phase cancellation by nearly axisymmetric accretion of the disrupted material. This feature is consistent with the formation of a massive remnant disk, which is described in Sec. IVB, for the prograde $\mathrm{BH}$ spin. For binaries with $(Q, a)=(3, \leq 0)$, on the other hand, gravitational waves end up with ringdown waveforms associated with the remnant $\mathrm{BHs}$ because the tidal effect is very weak throughout the merger. In these circumstances, gravitational waves do not show strong signatures of tidal deformation and disruption of the NS. 

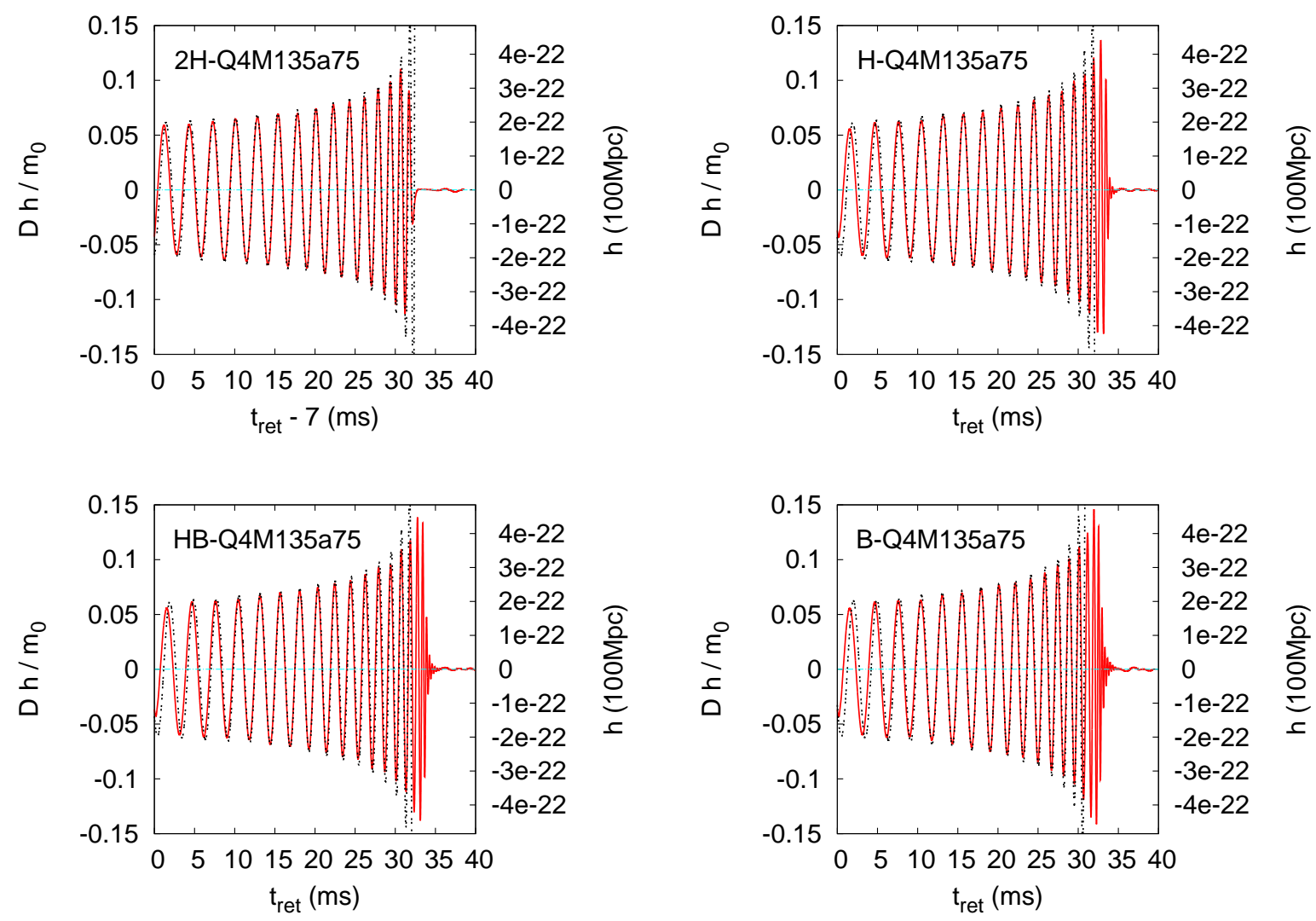

FIG. 15. The same as Fig. 14 but for models 2H-Q4M135a75, H-Q4M135a75, HB-Q4M135a75, and B-Q4M135a75.

Gravitational waves for a binary with $(Q, a)=(3,0.5)$ show a qualitatively new feature (the top right panel of Fig. 14). In this case, a ringdown waveform of the remnant $\mathrm{BH}$ is seen in the final stage, although the NS is tidally disrupted and the disk mass is larger than $0.1 M_{\odot}$. Namely, both tidal disruption of the NS and excitation of a QNM of the remnant $\mathrm{BH}$ occur in a compatible manner. The same feature is also found for a binary with a high mass ratio and a prograde $\mathrm{BH}$ spin, i.e., $(Q, a)=(\geq 4,0.75)$, shown in Figs. 15] and 16] except for $2 \mathrm{H} \mathrm{EOS}$, with which the NS is disrupted at a fairly distant orbit. These waveforms are often seen for BH-NS binaries with a heavy $\mathrm{BH}$ (or a high mass ratio) with the prograde $\mathrm{BH}$ spin, which results in the NS tidal disruption, and is never seen for BH-NS binaries with $Q=2$ or high mass-ratio binaries with nonspinning BHs.

The ratio of the areal radius of the remnant $\mathrm{BH}$ to the NS radius, $R_{\mathrm{NS}}$, is intimately related to the different excitation degree of the QNM between low and high mass-ratio binaries in the presence of NS tidal disruption [90, 91]. Schematic pictures of merger processes are depicted in Fig. 17. If tidal disruption does not occur, the NS is simply swallowed by the BH and excites a QNM, as shown in the middle panel of Fig. 17. If tidal disruption occurs in a binary with a low mass ratio, the disrupted material spreads around the $\mathrm{BH}$ to soon form a nearly axisymmetric disk. Approximately speaking, this occurs if the $\mathrm{BH}$ areal radius is smaller than the NS radius, as is shown in the left panel of Fig. 17. Thus, the NS tidal disruption has a strong effect to suppress the excitation of a QNM through the phase cancellation in the low mass-ratio binary. However, the situation is different in a high mass-ratio binary. Whereas the disrupted material forms an axisymmetric accretion disk around the $\mathrm{BH}$ in a sufficiently long time duration, the accretion just after the merger does not proceed in an axisymmetric way in high mass-ratio binaries, such as $Q=4$, except for the extremely stiff EOS. This is because the $\mathrm{BH}$ radius for $Q=4$ approximately doubles that for $Q=2$, and hence, the disrupted material takes longer time to spread around the $\mathrm{BH}$. In other words, the NS material accretes onto the $\mathrm{BH}$ coherently even after the tidal disruption, as is shown in the right panel of Fig. 17, because the $\mathrm{BH}$ radius is so large that the disrupted material cannot fully cover the $\mathrm{BH}$ surface before the $\mathrm{BH}$ swallows a large portion of the material. In the exceptional $2 \mathrm{H}$ EOS case, tidal disruption occurs sufficiently far outside the $\mathrm{BH}$ due to the large radius of the $\mathrm{NS}$, and hence, the disrupted material is able to spread around the $\mathrm{BH}$ to form a nearly axisymmetric accretion disk before the prompt infall. Therefore, the QNM of a remnant $\mathrm{BH}$ is not excited for $2 \mathrm{H}$ EOS. 

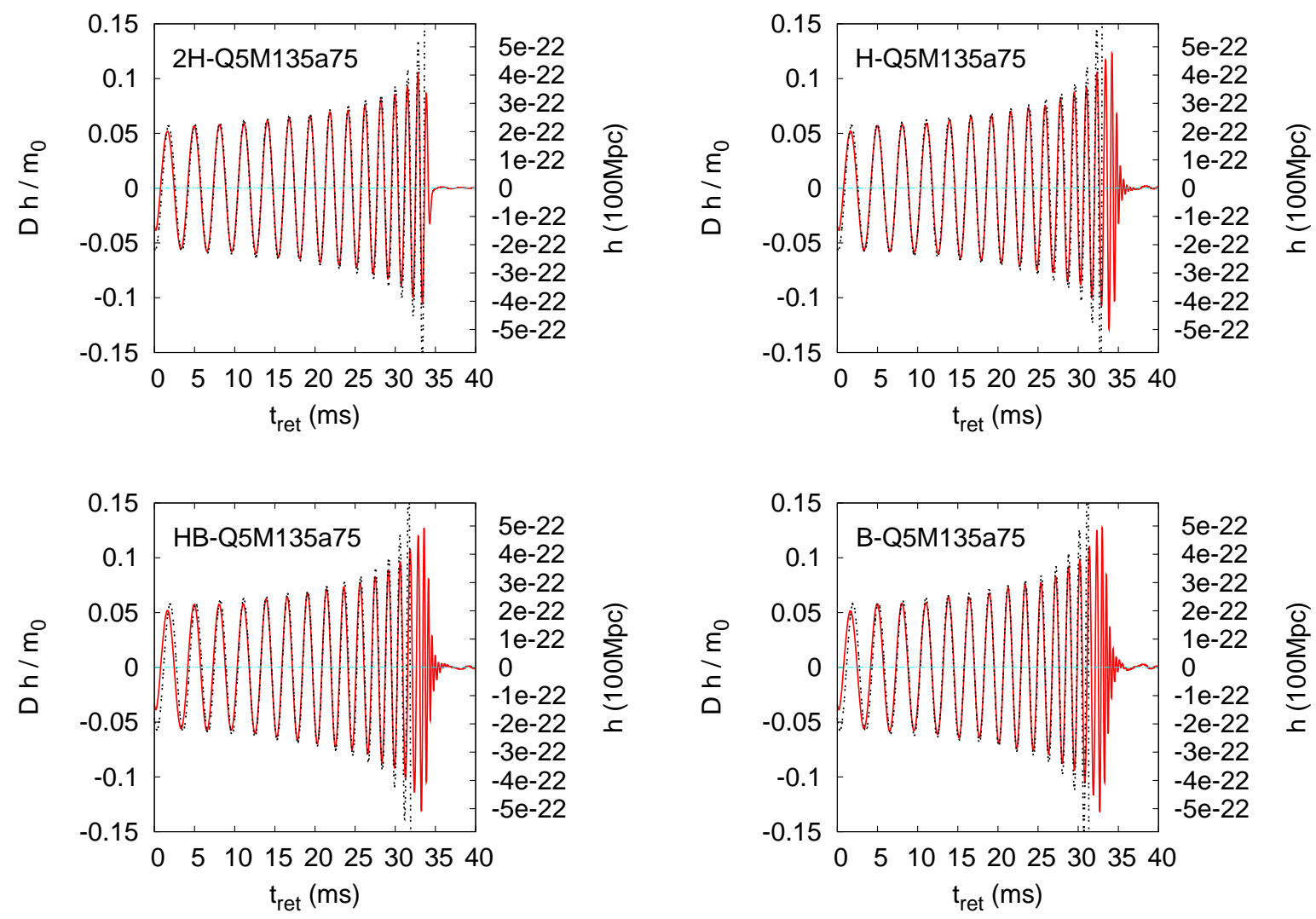

FIG. 16. The same as Fig. 14 but for models 2H-Q5M135a75, H-Q5M135a75, HB-Q5M135a75, and B-Q5M135a75.
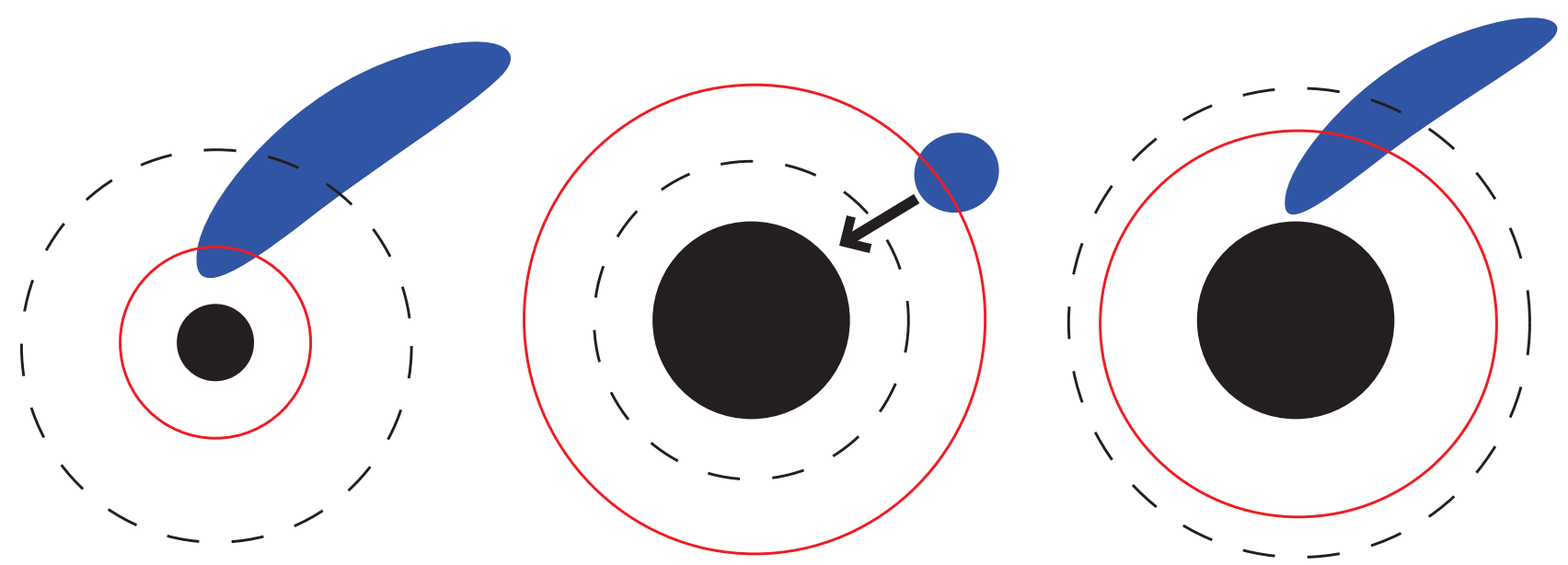

FIG. 17. Schematic pictures for three types of the merger process. The solid filled circle denotes the BH, the distorted ellipsoid denotes the NS, the solid circle is the location of the ISCO, and the dashed circle is the location of the radius at which the tidal disruption occurs. Left: the NS is tidally disrupted, and the spatial extent of the disrupted material is larger than or as large as that of the BH. Middle: the NS is not tidally disrupted. Right: the NS is tidally disrupted, and the spatial extent of the disrupted material is smaller than that of the $\mathrm{BH}$.

\section{F. Gravitational-wave spectrum}

Key features of gravitational waves are reflected in the Fourier spectrum. In this paper, we define the Fourier spectrum as a sum of each Fourier component of two 
independent polarizations of the $(l,|m|)=(2,2)$ mode as

$$
\begin{aligned}
\tilde{h}(f) & =\sqrt{\frac{\left|\tilde{h}_{+}(f)\right|^{2}+\left|\tilde{h}_{\times}(f)\right|^{2}}{2}}, \\
\tilde{h}_{A}(f) & =\int e^{2 \pi i f t} h_{A}(t) d t,
\end{aligned}
$$

where $A$ denotes two polarization modes, + or $\times$. We show a nondimensional spectrum, $f \tilde{h}(f)$, observed at a hypothetical distance of $100 \mathrm{Mpc}$ as a function of the gravitational-wave frequency, $f(\mathrm{~Hz})$, or a normalized amplitude, $f \tilde{h}(f) D / m_{0}$, as a function of a nondimensional frequency, $f m_{0}$. The amplitude of gravitational waves, $h_{A}$, is given as the amplitude observed along the $z$ axis, which is the most optimistic direction for the gravitational-wave detection. We note that the actual amplitude of gravitational waves depends on an angle locating the source in the sky and on an angle specifying the orientation of the orbital plane of the binary. The angular average of the effective amplitude is $\approx 0.4 f \tilde{h}(f)$. We always exclude spurious radiation components for $t_{\text {ret }} \lesssim 0 \mathrm{~ms}$, using a step function of the retarded time as a window function.

To show the dependence of the gravitational-wave spectra on the $\mathrm{BH}$ spin parameter, we plot the spectra for models HB-Q2M135a75, HB-Q2M135a5, HB-Q2M135, and HB-Q2M135a-5 in the left panel of Fig. 18 and for models HB-Q3M135a75, HB-Q3M135a5, HB-Q3M135, and HB-Q3M135a-5 in the right panel of Fig. 18. In the early inspiral phase of $f \lesssim 1 \mathrm{kHz}$, where the pointparticle approximation works well, the amplitude of the gravitational-wave spectrum for a given frequency increases monotonically as $a$ increases. This is a feature expected from the PN calculation and is explained by the spin-orbit interaction as follows: The power spectrum of gravitational radiation is written as

$$
\frac{d E}{d f} \propto[f \tilde{h}(f)]^{2}
$$

On the other hand, retaining only $1.5 \mathrm{PN}$, the lowestorder spin-orbit interaction terms, Eqs. (4.10) and (4.14) of Ref. [79], derive the expression for this quantity as

$$
\frac{d E}{d f}=\frac{Q}{3(1+Q)^{2}} \frac{X^{5 / 2}}{\pi f^{2}}\left[1+a X^{3 / 2}\left\{\frac{5(4 Q+3)}{3(1+Q)^{2}}\right\}\right] \text {. }
$$

Thus, the effective amplitude, $f \tilde{h}(f)$, for a given frequency $f$ increases monotonically as the $\mathrm{BH}$ spin parameter, $a$, increases in the inspiral phase.

Figure 19 plots the spectra for models 2H-Q2M135a75, H-Q2M135a75, HB-Q2M135a75, and B-Q2M135a75, for which only the EOS is different, and indicates that the amplitude in the early inspiral phase does not depend strongly on $\mathcal{C}$. This is because the finite-size effect of the NS does not play an important role in the early inspiral phase (but, see Refs. 93, 94]), as already found for nonspinning BH-NS binaries [31].
In the late inspiral phase of $1 \mathrm{kHz} \lesssim f \lesssim f_{\text {cut }}$, where $f_{\text {cut }}$ is a characteristic frequency at which the spectrum starts damping exponentially (see below), the amplitude is significantly larger than the Taylor-T4 formula for the cases in which the NS is not disrupted. This is because the binaries in the inspiral and plunge after the NS enters the BH's ISCO emit gravitational waves in reality, whereas the Taylor-T4 formula does not take into account the motion inside the ISCO. In contrast to the spectrum calculated by the Taylor-T4 formula, which decreases steeply after the last inspiral phase, the amplitude obtained from the simulation depends only weakly on the gravitational-wave frequency in that phase, as far as the tidal disruption does not occur.

The most fruitful information of the NS comes from the gravitational-wave spectrum in the merger phase through the "cutoff frequency," $f_{\text {cut }}$, which depends on the BH spin as well as the NS compactness [29, 31]. If the NS tidal disruption occurs, the spectrum damps at $f=f_{\text {tidal }} \sim 2-4 \mathrm{kHz}$, which denotes the frequency at the tidal disruption and depends sensitively on physical parameters of the binary. In that case, gravitational waves for a higher frequency, $f \gtrsim f_{\text {cut }} \approx f_{\text {tidal }}$, are not emitted by the binary in the inspiral motion but only weakly by disrupted material. Because the disrupted material gradually spreads around the $\mathrm{BH}$ to form a nearly axisymmetric disk, the emission of gravitational waves is suppressed at the high frequency. Thus, the spectrum shows a relatively moderate damping around $f \approx f_{\text {cut }}$, which is closely related to the NS compactness through the tidal disruption. The spectra for binaries with $(Q, a)=(2, \geq 0)$ and $(3, \geq 0.5)$ in the left panel of Fig. 18 correspond to these cases. We see that the cutoff frequency, $f_{\text {cut }}$, for these models decreases as the $\mathrm{BH}$ spin parameter increases. This is ascribed to the decrease of the orbital frequency at the tidal disruption for a binary with the prograde $\mathrm{BH}$ spin. The enhancement of the effective centrifugal force by the spin-orbit interaction reduces the orbital frequency at the tidal disruption, $f_{\text {tidal }}$, although the orbital separation at the tidal disruption itself does not vary much even in the presence of the $\mathrm{BH}$ spin. If the tidal disruption does not occur during the merger, however, inspiral-like motion continues at higher frequencies near and even inside the ISCO until the BH swallows the NS. In this case, the spectrum amplitude depends only weakly on $f$ in the frequency range $f \lesssim f_{\text {cut }}$ and damps for $f \gtrsim f_{\text {cut }}$, which is closely related to the QNM frequency of the remnant $\mathrm{BH}, f_{\mathrm{QNM}}$. The spectra for $(Q, a)=(2,-0.5)$ and $(3, \leq 0)$ in Fig. 18 show this feature. Note that the amplitude for model HB-Q3M135a-5 is smaller than for model HB-Q3M135 for the frequency range shown in Fig. 18 because tidal disruption does not play an important role, and Eq. (42) applies throughout the merger in both cases.

It is noteworthy that a prograde $\mathrm{BH}$ spin is favorable for the gravitational-wave detection in the inspiral phase and the estimation of $f_{\text {cut }}$ in the merger phase because the prograde spin enhances the amplitude for a given 

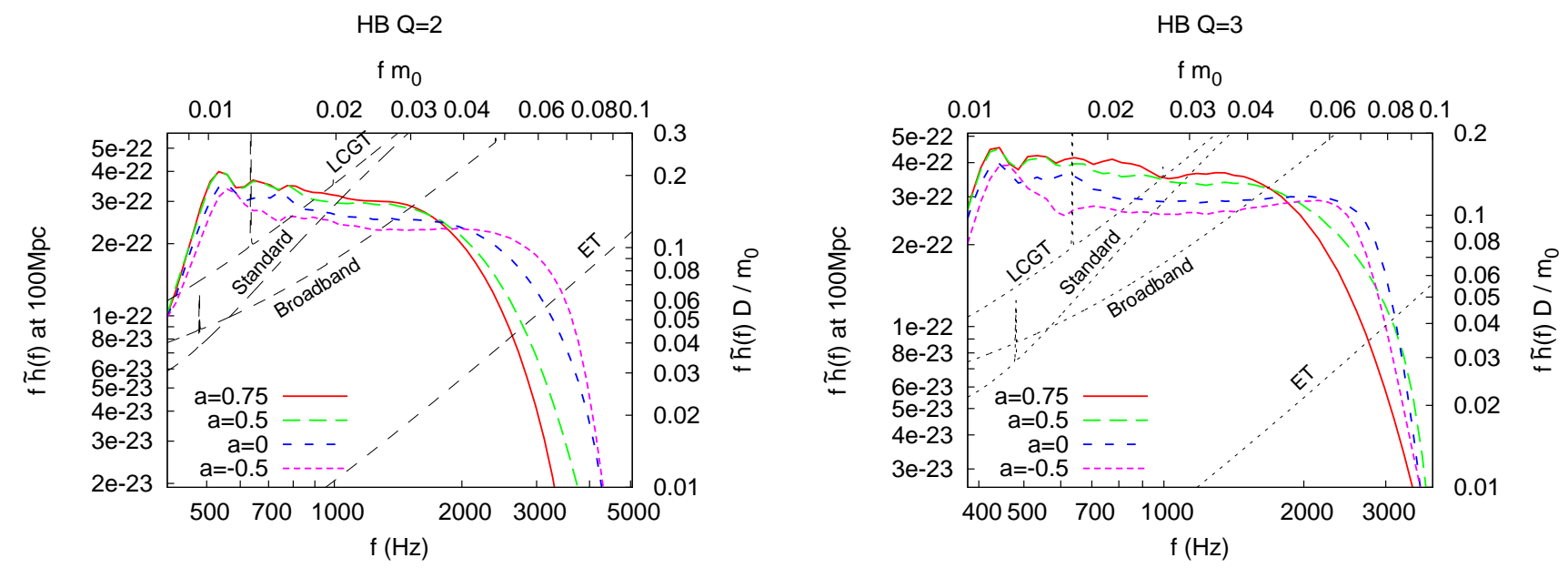

FIG. 18. Gravitational-wave spectra for BH-NS binaries with HB EOS, $M_{\mathrm{NS}}=1.35 M_{\odot}$ and $a=0.75,0.5,0$, and -0.5 . The left and right panels show the spectra for $Q=2$ and 3 , respectively. The upper axis denotes the normalized frequency, $f m_{0}$, and the right axis denotes the normalized amplitude, $f \tilde{h}(f) D / m_{0}$. The bottom axis denotes the frequency, $f$, in Hz, and the left axis denotes the nondimensional amplitude of gravitational waves, $f \tilde{h}(f)$, observed at a hypothetical distance $100 \mathrm{Mpc}$ from the binary along the $z$ axis. The dashed curves are planned noise curves of the LCGT ("LCGT"), the Advanced LIGO optimized for $1.4 M_{\odot}$ NS-NS detection ("Standard"), the Advanced LIGO optimized for the burst detection ("Broadband"), and the Einstein Telescope ("ET") 92 .

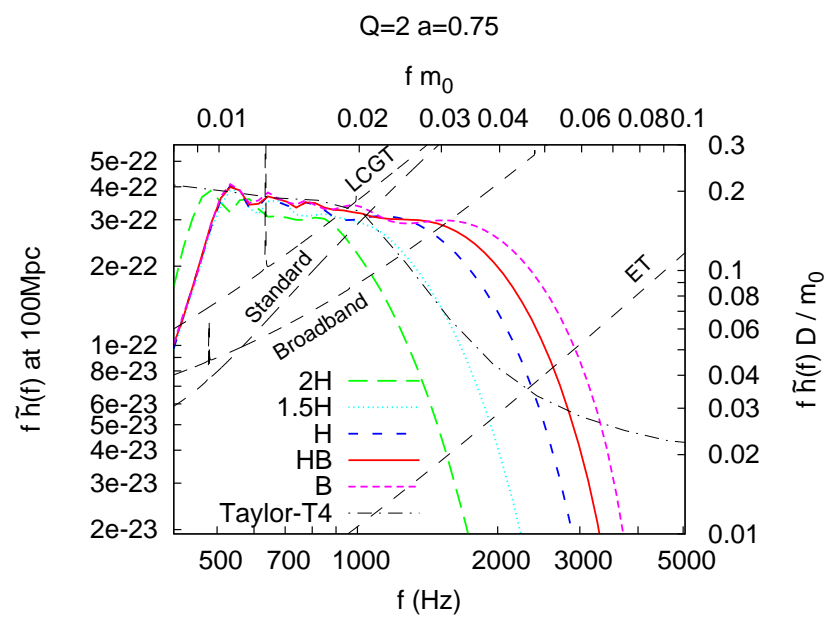

FIG. 19. The same as Fig. 18 but for $\left(Q, M_{\mathrm{NS}}, a\right)=$ $\left(2,1.35 M_{\odot}, 0.75\right)$ with $2 \mathrm{H}, \mathrm{H}, \mathrm{HB}$, and B EOSs. The spectrum derived by the Taylor-T4 formula is also included.

frequency in the inspiral phase and decreases the cutoff frequency in the merger phase. Note that the most sensitive frequency range for ground-based detectors is $f \sim 10$ $\mathrm{Hz}-1 \mathrm{kHz}$, which is usually lower than $f_{\text {cut }}$. Thus, the features found here are encouraging for the gravitationalwave astronomy to become an important tool for investigating the NS radius and EOS.

The gravitational-wave spectra of binaries with high mass ratios show qualitatively different behavior for a high frequency. Figure 20] plots the gravitationalwave spectra obtained for models with $\left(Q, M_{\mathrm{NS}}, a\right)=$ $\left(4,1.35 M_{\odot}, 0.75\right)$ and $\left(5,1.35 M_{\odot}, 0.75\right)$. For these bina- ries (except for the model with $2 \mathrm{H}$ EOS), both the NS tidal disruption and the excitation of a QNM of the remnant $\mathrm{BH}$ occur as is described in Sec. IVE. Hence, the gravitational spectrum has two characteristic frequencies, i.e., $f_{\text {tidal }}$ and $f_{\mathrm{QNM}}$, simultaneously. The spectra plotted in Fig. 20 indeed show such features. After the NS is tidally disrupted, the amplitude of the gravitationalwave spectrum shows a slow damp for $f \gtrsim f_{\text {tidal }} \approx 2 \mathrm{kHz}$. Then, the spectrum damps steeply above the frequency of the QNM, $f \gtrsim f_{\mathrm{QNM}} \approx 3 \mathrm{kHz}$. A schematic figure of different spectra is depicted in Fig. 21, and the spectrum described in this paragraph corresponds to spectrum (iii) in this figure. This suggests that the cutoff frequency, $f_{\text {cut }}$, of a high mass-ratio binary is not determined by a unique physical process like NS tidal disruption or a ringdown of a remnant $\mathrm{BH}$, as far as both of them occur.

To estimate the cutoff frequency quantitatively, we fit the gravitational-wave spectra by a function with seven free parameters of the form

$$
\begin{aligned}
\frac{f \tilde{h}_{\mathrm{fit}}(f) D}{m_{0}} & =\frac{f \tilde{h}_{3 \mathrm{PN}}(f) D}{m_{0}} e^{-\left(f / f_{\mathrm{ins}}\right)^{\sigma_{\mathrm{ins}}}} \\
& +A e^{-\left(f / f_{\mathrm{dam}}\right)^{\sigma_{\mathrm{dam}}}}\left[1-e^{-\left(f / f_{\mathrm{ins} 2}\right)^{\sigma_{\mathrm{ins} 2}}}\right]
\end{aligned}
$$

where $\tilde{h}_{3 \mathrm{PN}}(f)$ is the Fourier spectrum calculated by the Taylor-T4 formula. The first term in Eq. (43) models the inspiral spectrum, and the second term models the merger and ringdown spectra. We determine seven free parameters $f_{\text {ins }}, f_{\text {ins } 2}, f_{\text {dam }}, \sigma_{\text {ins }}, \sigma_{\text {ins } 2}, \sigma_{\text {dam }}$, and $A$ by the condition that the following weighted norm is minimized:

$$
\sum_{i}\left\{\left[f_{i} \tilde{h}_{i}\left(f_{i}\right)-f_{i} \tilde{h}_{\mathrm{fit}}\left(f_{i}\right)\right] f_{i}^{1 / 3}\right\}^{2}
$$



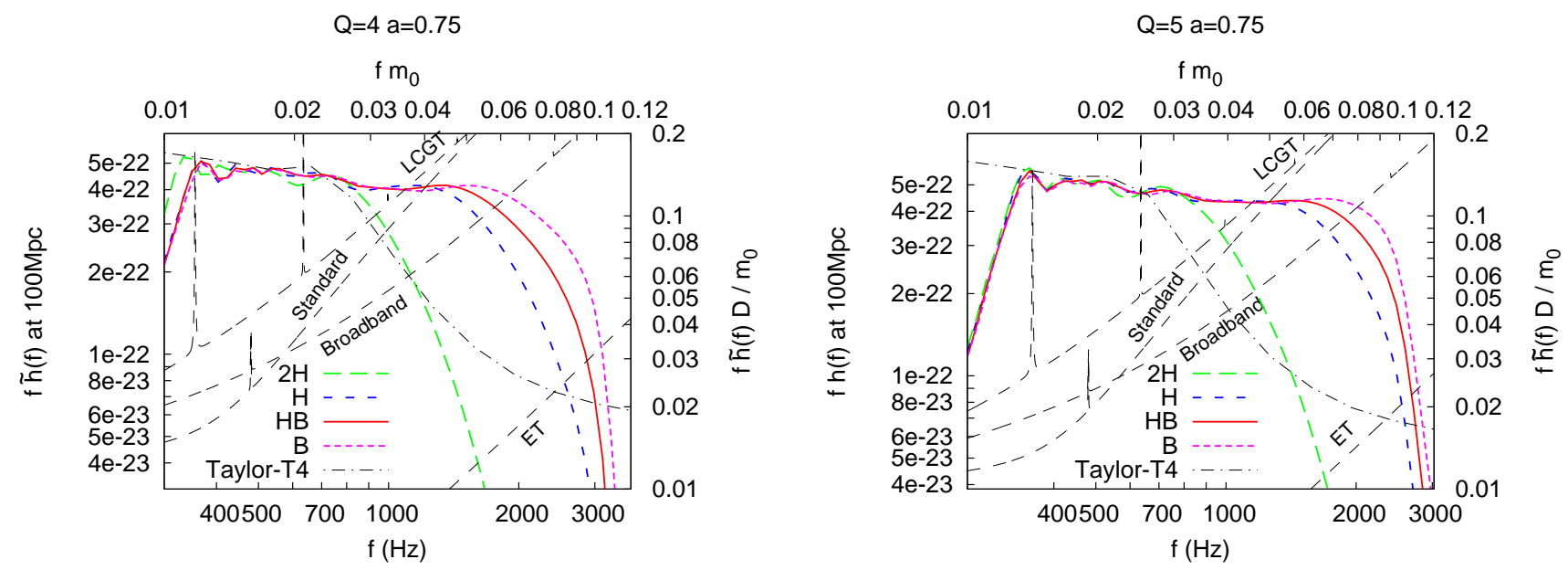

FIG. 20. The same as Fig. 18 but with the left panel for $\left(Q, M_{\mathrm{NS}}, a\right)=\left(4,1.35 M_{\odot}, 0.75\right)$ and the right panel for $\left(5,1.35 M_{\odot}, 0.75\right)$ with $2 \mathrm{H}, \mathrm{H}, \mathrm{HB}$, and B EOSs. The spectrum derived by the Taylor-T4 formula is also included.

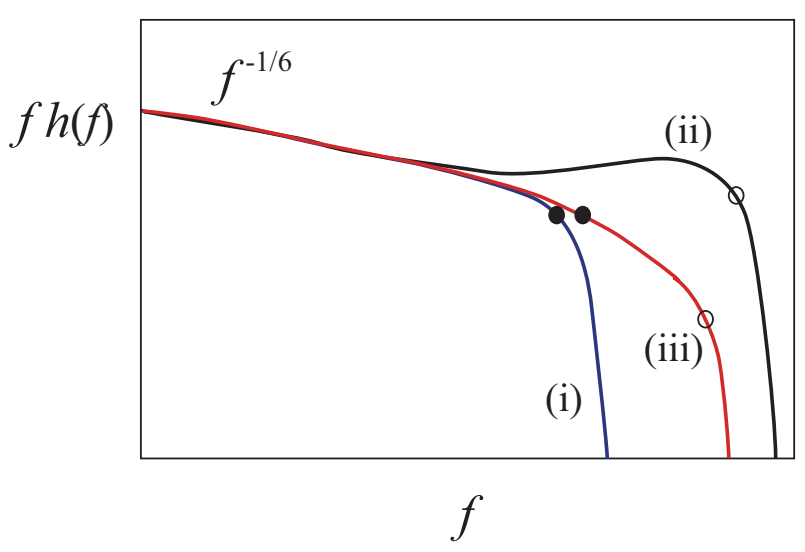

FIG. 21. A schematic figure of three types of gravitationalwave spectra. Spectrum (i) is for the case in which tidal disruption occurs far outside the ISCO, and spectrum (ii) is for the case in which tidal disruption does not occur. Spectrum (iii) is for the case in which tidal disruption occurs and the QNM is also excited. The filled and open circles denote $f_{\text {tidal }}$ and $f_{\mathrm{QNM}}$, respectively.

Here, $i$ denotes the data point for the spectrum. In the previous works 29, 31], we identify $f_{\text {dam }}$ in Eq. (43) with $f_{\text {cut }}$, which is most strongly correlated with the NS compactness for nonspinning BH-NS binaries [95]. In the present work, however, we obtain no strong correlation between $f_{\text {dam }}$ (and the other parameters) and any parameter of physical importance, such as $a$ or $\mathcal{C}$. The reason may be ascribed to the inadequacy of the functional form of Eq. (43), where the set of free parameters is degenerate to some extent. In particular, such a degeneracy is severe for a high mass-ratio binary due to two reasons. First, modeling an inspiral spectrum by the Taylor-T4 formula is inadequate for the late inspiral phase of a high mass-ratio binary due to the lack of information from the Taylor-T4 formula, as is described in Sec. IVE Second,

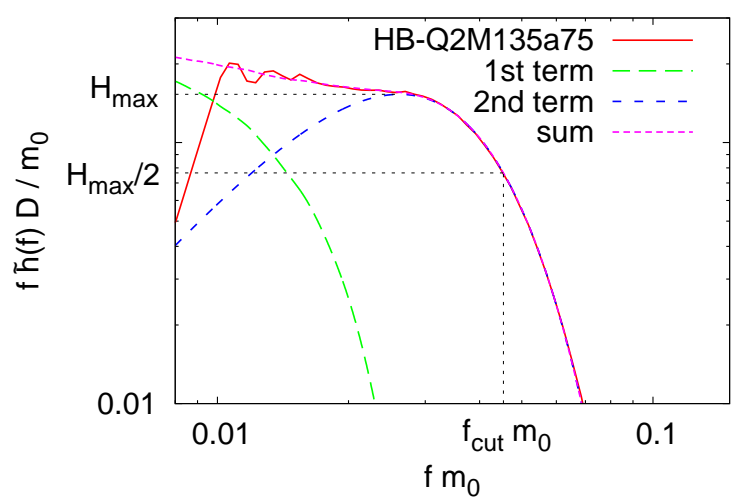

FIG. 22. The fitting for model HB-Q2M135a75. The longdashed and middle-dashed curves show the first and second terms of Eq. (43), respectively. The short-dashed curve is the sum of these two terms.

there is no unique, physically motivated identification of $f_{\text {cut }}$ when both the NS tidal disruption and the QNM excitation occur. (Fortunately, these degeneracies did not cause problems in the case of the nonspinning BH-NS binary with a low mass ratio [31].) To overcome these problems with the fitting procedure, we redefine $f_{\text {cut }}$ as the higher one of two frequencies at which the second term in Eq. (43) takes a half value of its maximum. An example of this fitting procedure is shown in Fig. 22, In this figure, $H_{\max }$ corresponds to the maximum value of the second term in Eq. (43). We find that this definition of $f_{\text {cut }}$ works well to read off the NS compactness from the gravitational-wave spectrum.

Figure 23 shows $f_{\text {cut }} m_{0}$ for spectra obtained for all binaries with $Q=2$ as a function of the NS compactness, $\mathcal{C}$, in logarithmic scales. We also plot the typical QNM frequency of the remnant $\mathrm{BH}, f_{\mathrm{QNM}}$, which depends primarily on $a$ of the initial $\mathrm{BH}$ for a fixed value of $Q$. For 


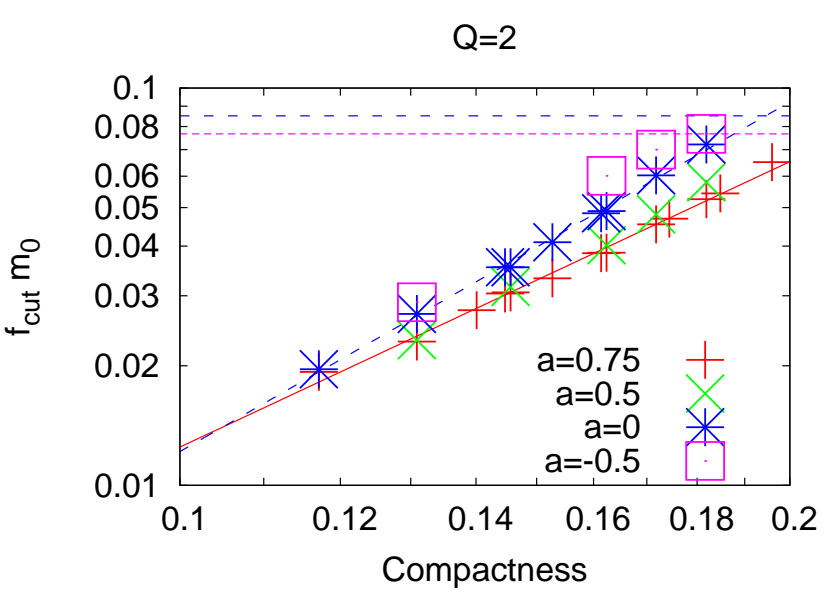

FIG. 23. The cutoff frequency times the total mass $f_{\text {cut }} m_{0}$ as a function of the NS compactness $\mathcal{C}$ for $Q=2$ binaries in logarithmic scales. The solid and dashed lines are obtained by linear fittings of data for $a=0.75$ and $a=0$, respectively. Horizontal lines denote the typical QNM frequencies of the remnant BHs. We note that $f_{\mathrm{QNM}}>f_{\text {cut }}$ for $a=0.75$ as long as $\mathcal{C} \leq 0.2$.

each value of $a$, we find that $f_{\text {cut }} m_{0}$ increases monotonically as $\mathcal{C}$ increases, and an approximate power law holds as

$$
\ln \left(f_{\text {cut }} m_{0}\right) \approx p(a) \ln \mathcal{C}+q(a) \quad(Q=2),
$$

where $p(a)(>0)$ and $q(a)$ depend only on $a$, for any value of $a$ when $Q=2$. This monotonic relation between $f_{\text {cut }} m_{0}$ and $\mathcal{C}$ suggests us a possibility to extract the compactness, $\mathcal{C}$, of a NS from the gravitational-wave observation. It is noteworthy that this relation includes only $\mathcal{C}$ but neither $M_{\mathrm{NS}}$ nor $R_{\mathrm{NS}}$ independently. It should also be noted that the simple relation found here is a consequence of our choice for a common value of the adiabatic index of the core EOS, $\Gamma_{2}=3$ [31]. The increase of $f_{\text {cut }} m_{0}$ with the increase of $\mathcal{C}$ indicates that a more compact NS is less subject to the BH tidal effect and disrupted at a closer orbit to the $\mathrm{BH}$ than a less compact NS is. The difference in $f_{\text {cut }} m_{0}$ due to the difference in $a$ becomes clearer for larger values of $\mathcal{C}$, and conversely, $f_{\text {cut }} m_{0}$ depends only weakly on $a$ if the compactness is as small as $\approx 0.12$. The weak dependence on $a$ for the small values of $\mathcal{C}$ is due to the fact that the effect of the $\mathrm{BH}$ spin at a distant orbit, at which the NS with a large radius is disrupted, is weak.

Figure 23 also shows that $p(a)$ is a decreasing function of $a$. More specifically we obtain the relations

$$
\ln \left(f_{\text {cut }} m_{0}\right)=(2.92 \pm 0.06) \ln \mathcal{C}+(2.32 \pm 0.12)
$$

for $a=0$ [96] and

$$
\ln \left(f_{\text {cut }} m_{0}\right)=(2.39 \pm 0.06) \ln \mathcal{C}+(1.11 \pm 0.11)
$$

for $a=0.75$ by a linear fitting. The decreasing nature of $p(a)$ is explained by the fact that the spin-orbit repulsive force for the prograde $\mathrm{BH}$ spin, which reduces the orbital frequency at the NS tidal disruption, works efficiently for a close orbit and hence for the NS with a large value of $\mathcal{C}$. It is important that $p(a)$ is always larger than 1.5, which is expected from the analysis of the condition for the mass shedding,

$$
\Omega m_{0} \propto \frac{\mathcal{C}^{3 / 2}(1+Q)^{3 / 2}}{\sqrt{Q}} .
$$

The large value of $p(a)$ is favorable for determining the NS compactness from the gravitational-wave observation because the dependence of $f_{\text {cut }}$ on $\mathcal{C}$ becomes stronger. Note that $f_{\text {cut }}$ is always lower than the QNM frequency of the remnant $\mathrm{BH}$ for a realistic range of the compactness $\mathcal{C} \lesssim 0.2$ for $(Q, a)=(2, \gtrsim 0)$. If $a$ is negative, on the other hand, $f_{\text {cut }}$ for the binary with a compact NS of $\mathcal{C} \gtrsim 0.18$ may be determined by the QNM frequency, $f_{\mathrm{QNM}}$, and it will be difficult to determine the NS compactness from the cutoff frequency.

Figure 24 shows the $f_{\text {cut }} m_{0}-\mathcal{C}$ relation of gravitationalwave spectra obtained for all binaries with $a=0.75$ and $a=0.5$. This figure, combined with Fig. 23, clearly indicates that the approximate power law of the form

$$
\ln \left(f_{\text {cut }} m_{0}\right)=p(Q, a) \ln \mathcal{C}+q(Q, a)
$$

holds for binaries of $\mathcal{C} \lesssim 0.2$ with $Q=5$ as far as $a \sim 0.75$ and with $Q \leq 4$ as far as $a \sim 0.5$. The striking feature is that the cutoff frequency is lower than the QNM frequency of the remnant BH, $f_{\mathrm{QNM}}$, for $(Q, a)=(\leq 4,0.75)$ and for $(Q, a)=(\leq 3,0.5)$ even if a QNM is excited. Accordingly, $f_{\text {cut }}$ shows a strong correlation with $\mathcal{C}$. For $(Q, a)=(5,0.75)$ and $(4,0.5), f_{\text {cut }}$ is lower than $f_{\mathrm{QNM}}$ as far as $\mathcal{C} \lesssim 0.18$ and 0.17 , respectively, and, therefore, the strong correlation between $f_{\text {cut }}$ and $\mathcal{C}$ is found within this range. Although $f_{\text {cut }}$ for the binary with a high mass ratio should not be considered as $f_{\text {tidal }}$ due to the QNM excitation, monotonic relations between $f_{\text {cut }} m_{0}$ and $\mathcal{C}$ gives us an opportunity to explore the NS radius and EOS. It should be noted that gravitational waves from a higher mass-ratio binary are more subject to the gravitationalwave detection due to the larger amplitude in the inspiral phase and the lower cutoff frequency. We again note that a massive $\mathrm{BH}$ of $M_{\mathrm{BH}} \gtrsim 5 M_{\odot}$ is an astrophysically realistic consequence of the stellar evolution [83, 84]. Taking these facts into account, we conclude that gravitational waves from the BH-NS binary are a promising tool to investigate the NS radius and EOS in the next decade.

\section{G. Energy and angular momentum radiated by gravitational waves}

Table VI lists the total energy $\Delta E / M_{0}$ and angular momentum $\Delta J / J_{0}$ radiated by gravitational waves. We estimate systematic errors in the estimation of $\Delta E$ and $\Delta J$ to be $\sim 10 \%$, which are ascribed mainly to the finite grid resolution and partly to the finite extraction radius 
$\mathrm{a}=0.75$

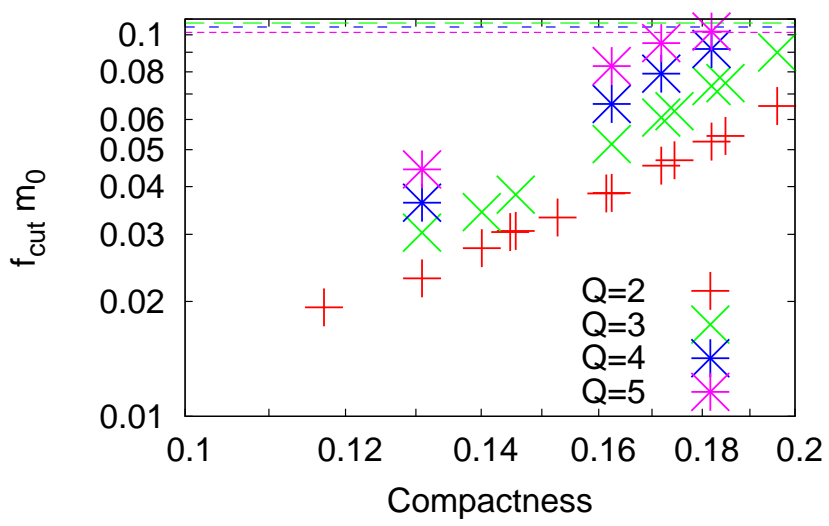

$\mathrm{a}=0.5$

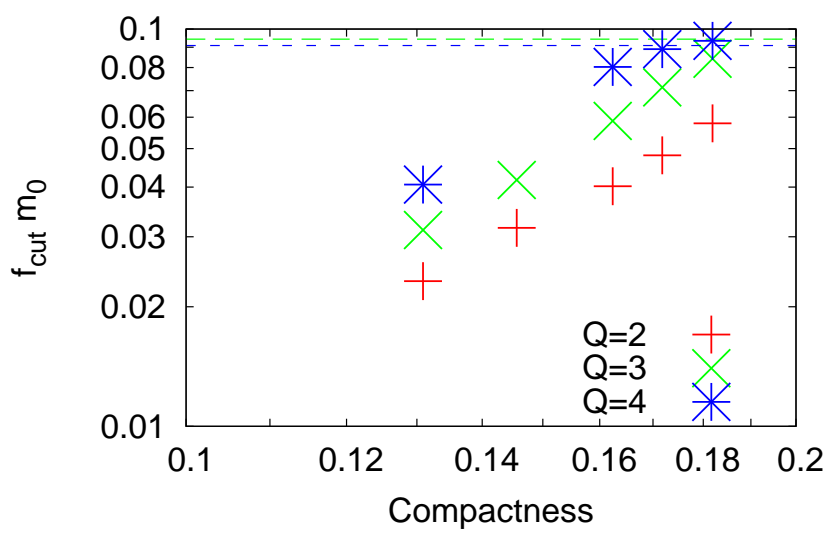

FIG. 24. The same as Fig. 23 but for $a=0.75$ (left) and 0.5 (right). In both panels, $f_{\mathrm{QNM}}>f_{\text {cut }}$ for $Q=2$.

for $\Psi_{4}$. Because $\Delta E$ and $\Delta J$ depend on the choice of $\Omega_{0} m_{0}$, we do not compare directly the results obtained for models with different values of $\Omega_{0} m_{0}$ and accordingly models with different values of $Q$ (see Table

Contributions from all the $l=2-4$ modes of gravitational waves are taken into account. The $(l,|m|)=(2,2)$ mode always contributes by $\gtrsim 85 \%$ to $\Delta E$ and $\Delta J$. $\Delta E$ and $\Delta J$ taken away by higher-mode gravitational waves are substantial for high mass-ratio binaries. For example, the $(3,3)$ mode contributes by $\sim 2,5,7.5$, and $10 \%$ for binaries with $Q=2,3,4$, and 5 , respectively. The $(4,4)$ mode gravitational waves contribute by $1 \sim 2 \%$ for binaries with $Q=3-5$. These values depend only weakly on $a$ and the EOS, and contributions of modes with $l \neq m$ are negligible compared to those of $l=m$ modes.

Table VI shows that $\Delta E / M_{0}$ and $\Delta J / J_{0}$ increase monotonically as the NS compactness, $\mathcal{C}$, increases for binaries with fixed values of $(Q, a)$. This is the same result as that obtained for nonspinning BH-NS binaries 31] and is explained by a longer inspiral phase for a softer EOS due to the later onset of mass shedding and the later tidal disruption. The ratio between these two values, $\left(\Delta J / J_{0}\right) /\left(\Delta E / M_{0}\right)$, decreases as $\mathcal{C}$ increases. This agrees again with the result for the nonspinning $\mathrm{BH}$ cases and is explained by a relation $\Delta J / \Delta E \approx m / \Omega$ for a given angular harmonic of $m$ and by the fact that more radiation is emitted from the orbit of a larger value of $\Omega$ for a softer EOS. Note that these arguments are based on little dependence of gravitational-wave luminosity in the inspiral phase on $\mathcal{C}$ for a fixed value of $a$; tidal correction to the luminosity in the inspiral phase is not important.

Table VI shows that $\Delta E / M_{0}$ does not depend strongly on $a$, while $\Delta J / J_{0}$ increases as $a$ increases in many cases for a fixed value of $\mathcal{C}$. Remember that $d E / d f$ in the inspiral phase increases for a large value of $a$, as is given by Eq. (42). However, the orbital frequency, $\Omega$, at the tidal disruption decreases for a large value of $a$ due to the spinorbit interaction. Because of these two competing effects, the binding energy at the tidal disruption depends only weakly on $a$, and hence, $\Delta E / M_{0}$ does not change very much among different values of $a$. The increase of $\Delta J / J_{0}$ for a large value of $a$ is due to the large value of $d E / d f$ in the inspiral phase, during which $\Omega$ is relatively low, and to the approximate relation $\Delta J \approx m \Delta E / \Omega$, which enhances the contribution of low-frequency gravitational waves.

Finally, we comment on the linear momentum $\Delta P$ radiated by gravitational waves and an associated kick velocity $v_{\text {kick }} \equiv \Delta P / M_{0}$ of the remnant $\mathrm{BH}$. Because of the mass and spin asymmetries, the remnant $\mathrm{BH}$ achieves the kick velocity of $\sim 100-250 \mathrm{~km} / \mathrm{s}$ when the effect of tidal disruption is weak, e.g., for models HB-Q3M135a5 and B-Q3M135. Although our results for $\Delta P$ do not converge as well as those for $\Delta E$ and $\Delta J$ due to the slow convergence of $(l, m) \neq(2,2)$ mode gravitational waves, the values of $v_{\text {kick }}$ are in reasonable agreements with the fitting formula derived using the results of simulations for the binary $\mathrm{BH}$ merger [97, 98]. By contrast, $v_{\text {kick }}$ is suppressed to $\lesssim 100 \mathrm{~km} / \mathrm{s}$ when tidal disruption occurs far outside the ISCO. The reason for this is that the tidal disruption suppresses significantly the gravitational radiation from the last inspiral and merger phases, during which the linear momentum is emitted most efficiently. This trend is consistent with the result found in our previous work [29].

\section{SUMMARY}

We performed numerical simulations for the merger of BH-NS binaries with various $\mathrm{BH}$ spins aligned or antialigned with the orbital angular momentum, using an AMR code SACRA with systematically chosen five piecewise polytropic EOSs. We investigated the dependence of the merger process, properties and structures of the remnant disk, properties of the remnant $\mathrm{BH}$, gravitational waveforms, and their spectra on the spin of the $\mathrm{BH}$ and the EOS of the NS. In particular, we focused on the case 
TABLE VI. Total radiated energy $\Delta E$ and angular momentum $\Delta J$ carried away by gravitational waves. $\Delta E$ and $\Delta J$ are normalized with respect to the initial ADM mass $M_{0}$ and angular momentum $J_{0}$, respectively. We also show the ratio between $\Delta J$ and $\Delta E$.

\begin{tabular}{|c|c|c|c|}
\hline Model & $\Delta E / M_{0}(\%)$ & $\Delta J / J_{0}(\%)$ & $\left(\Delta J / J_{0}\right) /\left(\Delta E / M_{0}\right)$ \\
\hline 2H-Q2M135a75 & 0.58 & 16 & 27 \\
\hline 1.5H-Q2M135a75 & 0.79 & 19 & 24 \\
\hline H-Q2M135a75 & 1.1 & 24 & 21 \\
\hline HB-Q2M135a75 & 1.4 & 26 & 19 \\
\hline B-Q2M135a75 & 1.7 & 29 & 17 \\
\hline 2H-Q2M135a5 & 0.60 & 17 & 26 \\
\hline 1.5H-Q2M135a5 & 0.79 & 19 & 24 \\
\hline H-Q2M135a5 & 1.2 & 24 & 20 \\
\hline HB-Q2M135a5 & 1.4 & 26 & 19 \\
\hline B-Q2M135a5 & 1.7 & 28 & 17 \\
\hline 2H-Q2M135a-5 & 0.57 & 15 & 26 \\
\hline H-Q2M135a-5 & 1.1 & 19 & 16 \\
\hline HB-Q2M135a-5 & 1.4 & 19 & 14 \\
\hline B-Q2M135a-5 & 1.6 & 21 & 13 \\
\hline 2H-Q2M12a75 & 0.40 & 12 & 30 \\
\hline H-Q2M12a75 & 0.79 & 19 & 24 \\
\hline HB-Q2M12a75 & 0.95 & 21 & 22 \\
\hline B-Q2M12a75 & 1.2 & 24 & 21 \\
\hline 2H-Q2M145a75 & 0.73 & 19 & 25 \\
\hline H-Q2M145a75 & 1.5 & 27 & 19 \\
\hline HB-Q2M145a75 & 1.7 & 30 & 17 \\
\hline B-Q2M145a75 & 2.1 & 32 & 15 \\
\hline 2H-Q3M135a75 & 0.72 & 20 & 28 \\
\hline 1.5H-Q3M135a75 & 0.97 & 23 & 24 \\
\hline H-Q3M135a75 & 1.3 & 27 & 20 \\
\hline HB-Q3M135a75 & 1.6 & 30 & 19 \\
\hline B-Q3M135a75 & 2.0 & 34 & 17 \\
\hline 2H-Q3M135a5 & 0.70 & 19 & 27 \\
\hline 1.5H-Q3M135a5 & 0.94 & 22 & 23 \\
\hline H-Q3M135a5 & 1.4 & 26 & 19 \\
\hline HB-Q3M135a5 & 1.7 & 29 & 17 \\
\hline B-Q3M135a5 & 2.0 & 31 & 15 \\
\hline HB-Q3M135a-5 & 1.3 & 19 & 14 \\
\hline 2H-Q3M145a75 & 0.88 & 22 & 25 \\
\hline H-Q3M145a75 & 1.7 & 31 & 18 \\
\hline HB-Q3M145a75 & 2.1 & 34 & 16 \\
\hline B-Q3M145a75 & 2.5 & 37 & 15 \\
\hline 2H-Q4M135a75 & 0.81 & 23 & 28 \\
\hline H-Q4M135a75 & 1.5 & 31 & 21 \\
\hline HB-Q4M135a75 & 1.8 & 33 & 19 \\
\hline B-Q4M135a75 & 2.1 & 36 & 17 \\
\hline 2H-Q4M135a5 & 0.72 & 19 & 27 \\
\hline H-Q4M135a5 & 1.5 & 27 & 19 \\
\hline HB-Q4M135a5 & 1.7 & 29 & 17 \\
\hline B-Q4M135a5 & 1.9 & 31 & 16 \\
\hline 2H-Q5M135a75 & 0.83 & 24 & 29 \\
\hline H-Q5M135a75 & 1.6 & 33 & 20 \\
\hline HB-Q5M135a75 & 1.9 & 35 & 19 \\
\hline B-Q5M135a75 & 2.1 & 36 & 17 \\
\hline
\end{tabular}

in which the $\mathrm{BH}$ has a prograde spin, and the tidal disruption of the NS by a companion $\mathrm{BH}$ plays an important role. We adopted a number of parameters for the mass ratio, NS mass, and $\mathrm{BH}$ spin. By preparing the initial condition with a distant orbit and a small eccentricity, we always tracked $\gtrsim 5$ quasicircular orbits in the inspiral phase and studied the merger phase in a realistic setting. The treatment of hydrodynamic equations and the estimation method of the disk mass are improved in this work. In the following, we summarize the conclusions in this paper:

1. It is shown that a prograde $\mathrm{BH}$ spin enhances the effect of NS tidal disruption by the spin-orbit interaction. The mass of the remnant disk increases as the $\mathrm{BH}$ spin increases because the ISCO radius of the $\mathrm{BH}$ becomes small. A remarkable point is that the BH-NS binary with a high mass ratio of even $Q=5$ can form a sufficiently massive disk of $\gtrsim 0.1 M_{\odot}$ for a wide range of the NS compactness if the $\mathrm{BH}$ has a prograde spin of $a=0.75$. This amount of the disk mass for a high mass-ratio binary is hardly expected if the $\mathrm{BH}$ is nonspinning. This fact suggests that the formation of a $\mathrm{BH}-$ massive accretion disk system is a frequent outcome of the BH-NS binary merger with a prograde $\mathrm{BH}$ spin and may be encouraging for the merger scenario of a short-hard GRB. By contrast, the disk mass becomes very small if the $\mathrm{BH}$ has a retrograde spin.

2. It is shown that some portion of the disrupted material can extend to $\gtrsim 400 \mathrm{~km}$ from the $\mathrm{BH}$ if the massive disk is formed. The maximum rest-mass density in the disk is larger for binaries with smaller values of $Q$ because the ISCO radius is smaller for them. The extent of the disk could be large for a large value of $Q$. For such a remnant disk, the lifetime should be longer.

3. The spin parameter of the remnant $\mathrm{BH}$ depends primarily on the spin parameter of the initial $\mathrm{BH}$, $a$, and the mass ratio, $Q$. In particular, extrapolation of our results suggests that the merger of an extremely spinning $\mathrm{BH}$ and an irrotational NS does not form an overspinning $\mathrm{BH}$.

4. The gravitational waveform also depends strongly on the BH spin. The number of gravitational-wave cycles becomes larger for a prograde $\mathrm{BH}$ spin than that for a zero BH spin in the inspiral phase because an additional repulsive force due to the spinorbit interaction reduces gravitational-wave luminosity and an approaching velocity of the binary. We found that the Taylor-T4 formula does not reproduce the phase evolution in the late inspiral phases accurately, especially when the mass ratio is large.

5. In our previous work for nonspinning BH-NS binaries, the waveforms are classified simply into two 
categories: when tidal disruption of the NS occurs, the waveform is composed of an inspiral waveform and a prompt shutdown at the tidal disruption. When tidal disruption does not occur, the waveform is composed of inspiral and QNM waveforms. However, we find that the NS tidal disruption and the excitation of a QNM can occur simultaneously for binaries with a high mass ratio and a prograde $\mathrm{BH}$ spin. This is because the disrupted material cannot become axisymmetric before the prompt infall due to a larger $\mathrm{BH}$ areal radius for a larger value of $Q$. As a result, the material accretes onto the remnant $\mathrm{BH}$ coherently, and, therefore, the QNM of the remnant $\mathrm{BH}$ is excited, except for the case in which the extremely stiff EOS is adopted.

6 . The cutoff frequency of the gravitational-wave spectrum is correlated with the NS compactness in a clear manner when the NS is disrupted, and the $\mathrm{BH}$ spin modifies this correlation. The prograde $\mathrm{BH}$ spin decreases the cutoff frequency for fixed values of $\mathcal{C}$ and $Q$ because the angular velocity at the tidal disruption becomes smaller than that for $a=0$. The cutoff frequency is lower for a smaller value of $\mathcal{C}$ for fixed values of $Q$ and $a$, as in the case of nonspinning BH-NS binaries, because the tidal effect is stronger and the disruption occurs at a more distant orbit. The BH spin also modifies the spectrum for the inspiral phase. Specifically, the spectrum amplitude for a given frequency in the inspiral phase becomes large when the $\mathrm{BH}$ has a prograde spin, and this is consistent with the $\mathrm{PN}$ estimation. Both the low cutoff frequency and large spectrum amplitude in the inspiral phase for a prograde BH spin are encouraging for gravitationalwave astronomy to become a tool to investigate the NS compactness and EOS. It is noteworthy that the BH-NS binary with a high mass ratio of $Q \gtrsim 5$ is a more promising target for ground-based gravitational-wave detectors if the $\mathrm{BH}$ has a prograde spin and the NS tidal disruption occurs.

Finally, we list several issues to be explored in the future. Piecewise polytropic EOSs with two pieces employed in this paper are not accurate enough to model high-mass NSs with large central density of $\rho_{\max } \gtrsim$ $10^{15} \mathrm{~g} / \mathrm{cm}^{3}$ [59]. More detailed (piecewise polytropic) EOSs are necessary to calculate gravitational waves from a BH-relatively massive NS binary merger for which the tidal deformation and disruption of the NS plays an important role, i.e., BH-NS binaries with moderately large BH spins of $a \gtrsim 0.75$. The implementation of detailed microphysics, such as a finite-temperature effect and a neutrino transport process, is essential even qualitatively to explore the evolution of the remnant $\mathrm{BH}$-accretion disk system and to discuss the jet launch such that shorthard GRBs require. Recently, Sekiguchi developed a code to perform fully general relativistic simulation with the finite-temperature EOS and an approximate neu- trino emission (the so-called leakage scheme) and succeed in simulating the stellar core collapse [99, 100] and the merger of binary NSs [101]. We plan to work on BH-NS binary mergers along these lines.

\section{ACKNOWLEDGMENTS}

K. K. is deeply grateful to Eric Gourgoulhon, Nicolas Vasset, and Benjamin D. Lackey for valuable discussion. We also thank Tetsuro Yamamoto for developing SACRA. Numerical computations of quasiequilibrium states were performed using the free library LORENE 33]. We thank members of the Meudon Relativity Group for developing LORENE. This work was supported by the Grant-in-Aid for Scientific Research (21340051), by the Grant-in-Aid for Scientific Research on Innovative Area (20105004), by the Grant-in-Aid for the Global COE Program "The Next Generation of Physics, Spun from Universality and Emergence," by the HPCI strategic program of Japanese MEXT, by the Grant-in-Aid for Research Activity Startup of JSPS (22840010), and by the Grant-in-Aid of JSPS.

\section{Appendix: Convergence of gravitational waves and the remnant disk mass}

This Appendix demonstrates that the convergence is approximately achieved for gravitational waves and masses of the remnant disks shown in Sec. IV. Figure 25 shows the evolution of the orbital angular velocity determined by Eq. (32) and gravitational waveforms obtained with different grid resolutions for models HB-Q4M135a75 and 2H-Q5M135a75. We perform an appropriate time shift in order to align the curves in the inspiral phase, and perform the rotation of + and $\times$ polarization modes of gravitational waveforms for $N=42$ and 36 . We note that the time to the merger, $t_{\text {merger }}$, is systematically longer for finer grid resolutions because numerical dissipation of the angular momentum is smaller [29, 31]. In both cases, the evolution of the orbital angular velocity, $\Omega(t)$, approximately agrees up to the merger, except for initial bursts associated with the junk radiation. The gravitational waveforms agree very well in the final $\sim 4$ orbits of the inspiral phase $(t \gtrsim 15 \mathrm{~ms})$, the merger phase, and the ringdown phase if the QNM is excited. By contrast, gravitational waveforms in the initial $\sim 2$ orbits depend strongly on the grid resolutions, because the early inspiral phase is strongly affected by the dissipation of the junk radiation. We conclude that the convergence is approximately achieved for gravitational waves in the late inspiral, merger, and ringdown phases.

Figure 26] shows the evolution of the rest mass located outside the AH, $M_{r>r_{\mathrm{AH}}}$, with different grid resolutions for models HB-Q4M135a75 and 2H-Q5M135a75. This figure shows that the convergence is also approximately achieved for the mass of the remnant disk. Quantitatively, differences in $M_{r>r_{\mathrm{AH}}}$ at $\approx 10 \mathrm{~ms}$ after the merger 
are $\approx 2.9 \%$ and $\approx 2.6 \%$ for models HB-Q4M135a75 and 2H-Q5M135a75, respectively. If we assume the first-order convergence for $M_{r>r_{\mathrm{AH}}}$, the errors in the values obtained for $N=50$ runs are $\approx 7.5 \%$ and $\approx 6.8 \%$ for models HBQ4M135a75 and 2H-Q5M135a75, respectively.
[1] B. P. Abbott et al., Rep. Prog. Phys. 72, 076901 (2009).

[2] T. Accadia et al., Class. Quantum Grav. 28, 025005 (2011).

[3] K. Kuroda and the LCGT Collaboration, Class. Quantum Grav. 27, 084004 (2010).

[4] L. Lindblom, Astrophys. J. 398, 569 (1992).

[5] M. Vallisneri, Phys. Rev. Lett. 84, 3519 (2000).

[6] J. S. Read, C. Markakis, M. Shibata, K. Uryū, J. D. E. Creighton, and J. L. Friedman, Phys. Rev. D 79, 124033 (2009).

[7] V. Ferrari, L. Gualtieri, and F. Pannarale, Phys. Rev. D 81, 064026 (2010).

[8] F. Pannarale, A. Tonita, and L. Rezzolla, Astrophys. J. 727, 95 (2011).

[9] F. Pannarale, L. Rezzolla, F. Ohme, and J. S. Read, arXiv:1103.3526.

[10] P. Demorest, T. Pennucci, S. Ransom, M. Roberts, and J. Hessels, Nature 467, 1081 (2010).

[11] A. W. Steiner, J. M. Lattimer, and E. F. Brown, Astrophys. J. 722, 33 (2010).

[12] E. Nakar, Phys. Rep. 442, 166 (2007).

[13] W. H. Lee and E. Ramirez-Ruiz, New J. Phys 9, 17 (2007).

[14] R. D. Blandford and R. D. Znajek, Mon. Not. Roy. Astron. Soc. 179, 433 (1977).

[15] E. Berger et al,, Nature 438, 988 (2005).

[16] W. Fong, E. Berger, R. Chornock, N. R. Tanvir, A. J. Levan, J. F. Graham, A. S. Fruchter, A. Cucchiara, and D. B. Fox, Astrophys. J. 730, 26 (2011).

[17] S. E. Woosley, Astrophys. J. 405, 273 (1993).

[18] P. Grandclément, Phys. Rev. D 74, 124002 (2006); 75, 129903(E) (2007).

[19] K. Taniguchi, T. W. Baumgarte, J. A. Faber, and S. L. Shapiro, Phys. Rev. D 75, 084005 (2007).

[20] K. Taniguchi, T. W. Baumgarte, J. A. Faber, and S. L. Shapiro, Phys. Rev. D 77, 044003 (2008).

[21] F. Foucart, L. E. Kidder, H. P. Pfeiffer, and S. A. Teukolsky, Phys. Rev. D 77, 124051 (2008).

[22] K. Kyutoku, M. Shibata, and K. Taniguchi, Phys. Rev. D 79, 124018 (2009).

[23] M. Shibata and K. Uryū, Phys. Rev. D 74, 121503(R) (2006).

[24] M. Shibata and K. Uryū, Class. Quant. Grav. 24, S125 (2007).

[25] M. Shibata and K. Taniguchi, Phys. Rev. D 77, 084015 (2008).

[26] Z. B. Etienne, J. A. Faber, Y. T. Liu, S. L. Shapiro, K. Taniguchi, and T. W. Baumgarte, Phys. Rev. D 77, 084002 (2008).

[27] M. D. Duez, F. Foucart, L. E. Kidder, H. P. Pfeiffer, M. A. Scheel, and S. A. Teukolsky, Phys. Rev. D 78, 104015 (2008).

[28] Z. B. Etienne, Y. T. Liu, S. L. Shapiro, and T. W. Baumgarte, Phys. Rev. D 79, 044024 (2009).

[29] M. Shibata, K. Kyutoku, T. Yamamoto, and K. Taniguchi, Phys. Rev. D 79, 044030 (2009).
[30] M. D. Duez, F. Foucart, L. E. Kidder, C. D. Ott, and S. A. Teukolsky, Class. Quant. Grav. 27, 114106 (2010).

[31] K. Kyutoku, M. Shibata, and K. Taniguchi, Phys. Rev, D 82, 044049 (2010).

[32] S. Chawla, M. Anderson, M. Besselman, L. Lehner, S. L. Liebling, P. M. Motl, and D. Neilsen, Phys. Rev. Lett. 105, 111101 (2010).

[33] LORENE website, http://www . lorene.obspm.fr/.

[34] G. B. Cook, Living Rev. Relativity 3, 5 (2000).

[35] L. Bildsten and C. Cutler, Astrophys. J. 400, 175 (1992).

[36] C. S. Kochanek, Astrophys. J. 398, 234 (1992).

[37] J. W. York, Phys. Rev. Lett. 82, 1350 (1999).

[38] H. P. Pfeiffer and J. W. York, Phys. Rev. D 67, 044022 (2003).

[39] S. Brandt and B. Brügmann, Phys. Rev. Lett. 78, 3606 (1997).

[40] M. Campanelli, C. O. Lousto, P. Marronetti, and Y. Zlochower, Phys. Rev. Lett. 96, 111101 (2006).

[41] J. G. Baker, J. Centrella, D.-I. Choi, M. Koppitz, and J. van Meter, Phys. Rev. Lett. 96, 111102 (2006).

[42] R. Beig, Phys. Lett. A 69, 153 (1978).

[43] A. Ashtekar and A. Magnon-Ashtekar, J. Math. Phys. 20, 793 (1979).

[44] J. M. Bowen and J. W. York, Phys. Rev. D 21, 2047 (1980).

[45] A. Ashtekar and B. Krishnan, Living Rev. Relativity 7, 10 (2004).

[46] E. Gourgoulhon and J. L. Jaramillo, Phys. Rep. 423, 159 (2006).

[47] L.-M. Lin and J. Novak, Class. Quant. Grav. 24, 2665 (2007).

[48] G. B. Cook and B. F. Whiting, Phys. Rev. D 76, 041501 (2007).

[49] G. Lovelace, R. Owen, H. P. Pfeiffer, and T. Chu, Phys. Rev. D 78, 084017 (2008).

[50] S. Dain, C. O. Lousto, and Y. Zlochower, Phys. Rev. D 78, 024039 (2008).

[51] S. Bonazzola, E. Gourgoulhon, and J.-A. Marck, Phys. Rev. D 56, 7740 (1997).

[52] H. Asada, Phys. Rev. D 57, 7292 (1998).

[53] M. Shibata, Phys. Rev. D 58, 024012 (1998).

[54] S. A. Teukolsky, Astrophys. J. 504, 442 (1998).

[55] G. Faye, L. Blanchet, and A. Buonanno, Phys. Rev. D 74, 104033 (2006).

[56] L. Blanchet, A. Buonanno, and G. Faye, Phys. Rev. D 74, 104034 (2006).

[57] L. Blanchet, Phys. Rev. D 65, 124009 (2002).

[58] J. M. Lattimer and M. Prakash, Science 304, 536 (2004).

[59] J. S. Read, B. D. Lackey, B. J. Owen, and J. L. Friedman, Phys. Rev. D 79, 124032 (2009).

[60] I. H. Stairs, Science 304, 547 (2004).

[61] J. M. Lattimer and M. Prakash, Astrophys. J. 550, 426 (2001).

[62] In this paper, we determine the stiffness simply as the 

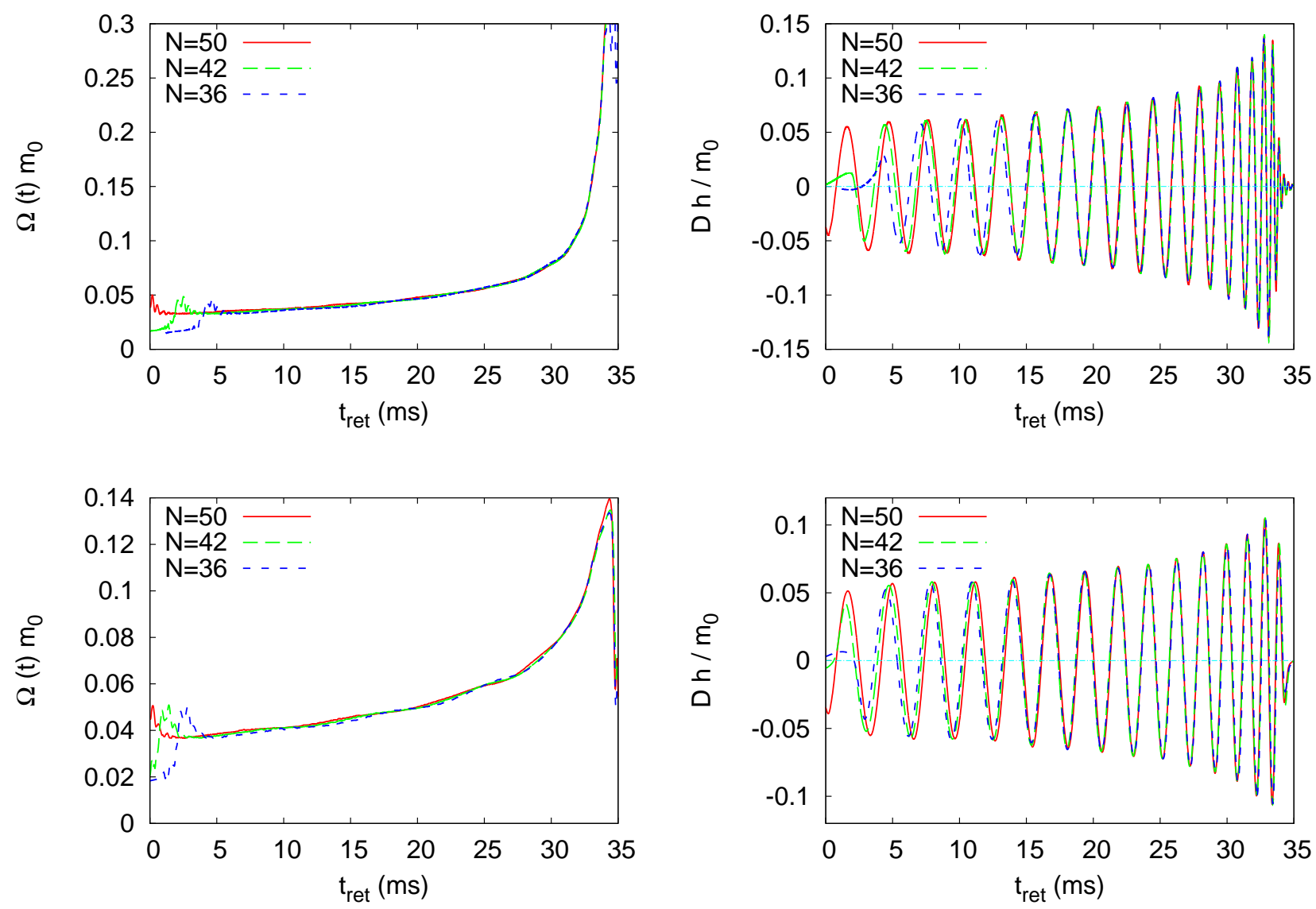

FIG. 25. Comparisons of evolution of the orbital angular velocity and gravitational waveforms for models HB-Q4M135a75 (top) and 2H-Q5M135a75 (bottom) with appropriate time shifts.

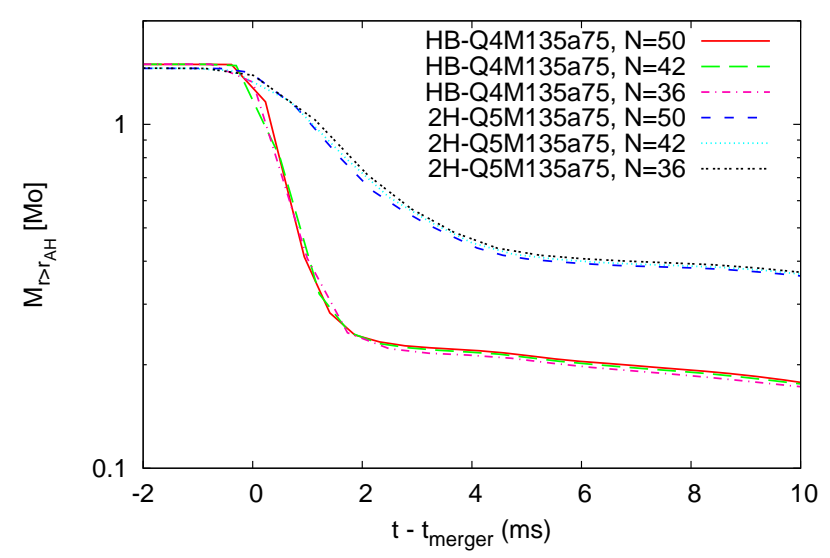

FIG. 26. Comparisons of evolution of the remnant disk masses for models HB-Q4M135a75 and 2H-Q5M135a75.

magnitude of the pressure for the nuclear-density region.

[63] B. D. Lackey, K. Kyutoku, M. Shibata, P. R. Brady, and J. L. Friedman, to be published.

[64] M. Shibata, Prog. Theor. Phys. 96, 917 (1996).
[65] P. Wiggins and D. Lai, Astrophys. J. 532, 530 (2000).

[66] In this paper, "the ISCO radius" always represents "the ISCO radius in the Boyer-Lindquist coordinates," which is physical in the sense that it gives the proper circumferential length for the equatorial circular orbit. It should be noted that the coordinate radius of the ISCO in our simulation is different from the Boyer-Lindquist one.

[67] J. M. Bardeen, W. H. Press, and S. A. Teukolsky, Astrophys. J. 178, 347 (1972).

[68] L. Blanchet, Living Rev. Relativity 9, 4 (2006).

[69] T. Yamamoto, M. Shibata, and K. Taniguchi, Phys. Rev. D 78, 064054 (2008).

[70] M. Shibata and T. Nakamura, Phys. Rev. D 52, 5428 (1995).

[71] T. W. Baumgarte and S. L. Shapiro, Phys. Rev. D 59, 024007 (1998).

[72] B. Brügmann, J. A. González, M. Hannam, S. Husa, U. Sperhake, and W. Tichy, Phys. Rev. D 77, 024027 (2008).

[73] A. Kurganov and E. Tadmor, J. Comput. Phys. 160, 241 (2000).

[74] C. Reisswig and D. Pollney, arXiv:1006.1632.

[75] M. Boyle, D. A. Brown, L. E. Kidder, A. H. Mroué, H. P. Pfeiffer, M. A. Scheel, G. B. Cook, and S. A. Teukolsky, Phys. Rev. D 76, 124038 (2007). 
[76] L. Santamaria, F. Ohme, P. Ajith, B. Brügmann, N. Dorband, M. Hannam, S. Husa, P. Mösta, D. Pollney, C. Reisswig, E. L. Robinson, J. Seiler, and B. Krishnan, Phys. Rev. D 82, 064016 (2010).

[77] L. E. Kidder, Phys. Rev. D 77, 044016 (2008).

[78] T. Damour and A. Nagar, arXiv:0906.1769.

[79] L. E. Kidder, Phys. Rev. D 52, 821 (1995).

[80] L. G. Fishbone, Astrophys. J. 185, 43 (1973).

[81] J.-A. Marck, Proc. Roy. Soc. London 385, 431 (1983).

[82] M. Ishii, M. Shibata, and Y. Mino, Phys. Rev. D 71, 044017 (2005).

[83] J. E. McClintock and R. A. Remillard, in Compact Stellar X-ray Sources, edited by W. H. G. Lewin and M. van der Klis (Cambridge University Press, 2006).

[84] K. Belczynski, T. Bulik, C. L. Fryer, A. Ruiter, F. Valsecchi, J. S. Vink, and J. R. Hurley, Astrophys. J. 714, 1217 (2010).

[85] F. Foucart, M. D. Duez, L. E. Kidder, and S. A. Teukolsky, Phys. Rev. D 83, 024005 (2011).

[86] R. Penrose, in general relativity: an Einstein centenary survey, edited by S. W. Hawking and W. Israel (1979).

[87] E. Berti, V. Cardoso, and A. O. Starinets, Class. Quantum Grav. 26, 163001 (2009).

[88] K. Uryū, F. Limousin, J. L. Friedman, E. Gourgoulhon, and M. Shibata, Phys. Rev. Lett. 97, 171101 (2006).

[89] K. Uryū, F. Limousin, J. L. Friedman, E. Gourgoulhon, and M. Shibata, Phys. Rev. D 80, 124004 (2009).

[90] M. Saijo and T. Nakamura, Phys. Rev. Lett. 85, 2665 (2000).

[91] M. Saijo and T. Nakamura, Phys. Rev. D 63, 064004 (2001).

[92] M. Punturo et al., Class. Quantum Grav. 27, 194002 (2010).

[93] L. Baiotti, T. Damour, B. Giacomazzo, A. Nagar, and L. Rezzolla, Phys. Rev. Lett. 105, 261101 (2010).

[94] L. Baiotti, T. Damour, B. Giacomazzo, A. Nagar, and L. Rezzolla, Phys. Rev. D 84, 024017 (2011).

[95] We refer to $f_{\text {dam }}$ as $f_{\text {cut }}$ throughout in the previous work [31]. In the present paper, we distinguish $f_{\text {dam }}$ from $f_{\text {cut }}$ because the method for determining $f_{\text {dam }}$ is different from that for $f_{\text {cut }}$.

[96] The relation between $f_{\text {cut }} m_{0}$ and $\mathcal{C}$ is different from the one obtained in Ref. [31] due to the different definition of $f_{\text {cut }}$.

[97] L. Rezzolla, Class. Quantum Grav. 26, 094023 (2009).

[98] Y. Zlochower, M. Campanelli, and C. O. Lousto, Class. Quantum Grav. 28, 114015 (2011).

[99] Y. Sekiguchi, Prog. Theor. Phys. 124, 331 (2010).

[100] Y. Sekiguchi and M. Shibata, Astrophys. J. 737, 6 (2011).

[101] Y. Sekiguchi, K. Kiuchi, K. Kyutoku, and M. Shibata, Phys. Rev. Lett. 107, 051102 (2011). 